\title{
The Scanning Transmission X-Ray Microscope at BESSY II
}

\author{
Dissertation \\ zur Erlangung des Doktorgrades \\ der Mathematisch-Naturwissenschaftlichen Fakultäten \\ der Georg-August-Universität zu Göttingen
}

vorgelegt von

Urs Wiesemann

aus Göttingen

Göttingen 2003 
Referent: Prof. Dr. G. Schmahl

Korreferent: Prof. Dr. R. Kirchheim

Tag der mündlichen Prüfung: 09.12. 2003 


\section{Contents}

$\begin{array}{lr}\text { Introduction } & 1\end{array}$

1 X-Ray Microscopy $\quad 3$

1.1 Interaction of Soft X-rays With Matter . . . . . . . . . . . 3

1.1.1 X-ray Spectroscopy for Elemental and Chemical Mapping . $\quad 5$

1.2 Transmission Zone Plates as High Resolution X-Ray Optics . . . . 6

1.3 Transmission X-Ray Microscopes (TXMs) and Scanning Transmission X-Ray Microscopes (STXMs) . . . . . . . . . . . . 8

1.4 Image Formation in the STXM . . . . . . . . . . . . . . . 10

1.4.1 Imaging With a Configured Detector . . . . . . . . . . . . 14

1.4.2 Contrast and Positioning Noise . . . . . . . . . . . . . 16

1.4.3 Signal-to-Noise Ratio and Photon Numbers . . . . . . . 17

2 The STXM at the Undulator U41 at BESSY II 21

3 The STXM Monochromator $\quad 25$

3.1 The Undulator U41 . . . . . . . . . . . . . . . . . . . . 25

3.2 Principle of Operation of the Monochromator . . . . . . . . . 31

3.2.1 The Diffraction Grating . . . . . . . . . . . . . 32

3.2.2 Spatial Coherence of the Zone Plate Illumination . . . . . 35

3.2.3 Spectral Contamination by Higher Undulator Harmonics . 35

3.3 The STXM Beamline . . . . . . . . . . . . . . . 36

3.3.1 The Beam Monitor . . . . . . . . . . . . . . 38

3.4 Mechanical Setup of the Monochromator . . . . . . . . . . . 38

3.4.1 The Principle of the Mirror Motion . . . . . . . . . . . . 40

3.4.2 The Alignment of the Monochromator . . . . . . . . . 43

3.5 Characterization of the Monochromator . . . . . . . . . . . . . 44

3.5.1 Photon Rate. . . . . . . . . . . . . . . . . . . 45

3.5.2 Measurement of the Spectral Resolution . . . . . . . . . . . 47

3.5.3 Energy Reproducibility . . . . . . . . . . . . . . . . . 48

3.5.4 Measurement of Higher Harmonics Radiation . . . . . . . 48

3.6 Summary . . . . . . . . . . . . . . . . . . . . . . . 49 
4 The Object Stage $\quad 51$

4.1 Image Field Limitation by the OSA and the Central Stop . . . . . 51

4.2 Zone Plates for the STXM . . . . . . . . . . . . . 53

4.3 Mechanical Setup of the Object Stage . . . . . . . . . . . . 55

4.3.1 Alignment of the Optical Elements . . . . . . . . . . 59

4.4 The Zone Plate Scanning Stage . . . . . . . . . . . . . . . . 60

4.4.1 Test of the Stage Accuracy in Motion . . . . . . . . . . . 60

4.5 The Wet Specimen Chamber . . . . . . . . . . . . . . . 62

5 The STXM detector $\quad \mathbf{6 5}$

5.1 Operating Principle of the Charge Coupled Device . . . . . . . . 66

5.2 The pn-CCD Detector . . . . . . . . . . . . . . . . 68

5.2.1 The pn-CCD Detector Used for the BESSY II STXM . . . 69

5.3 The Front End Electronics for the pn-CCD . . . . . . . . . . 72

5.3 .1 Processing of the pn-CCD data . . . . . . . . . . 75

5.4 Characterization of the PN-CCD Detector . . . . . . . . . . 76

5.4.1 Detector Calibration with a $\mathrm{Fe}^{55}$ source . . . . . . . . 76

5.4.2 Tests with Synchrotron Radiation . . . . . . . . . 78

6 First Measurements with the STXM

6.1 Spatial resolution of the STXM images . . . . . . . . . . . . . 85

6.2 Elemental Contrast Images . . . . . . . . . . . . . . . . . . 87

6.3 Differential Phase Contrast Images . . . . . . . . . . . . . . . . 89

$\begin{array}{ll}\text { Conclusion and Outlook } & 93\end{array}$

$\begin{array}{lr}\text { A Computer Control of the STXM } & 97\end{array}$

A.1 RT-Linux . . . . . . . . . . . . . . . . . . . . . . . 98

A.2 PID Control . . . . . . . . . . . . . . . . . . 101

B The Vacuum Window and Zone Plate Holders 103

B.1 The Zone Plate Holder . . . . . . . . . . . . . . . . . . . . . . . 103

B.2 The Vacuum Windows and Holders . . . . . . . . . . . . . . . . 104

$\begin{array}{ll}\text { Bibliography } & 107\end{array}$ 


\section{List of Figures}

1.1 Absorption length $\mu^{-1}$ of water, protein, and clay . . . . . . . 4

1.2 Schematic electron potential and K-shell spectrum of a diatomic molecule . . . . . . . . . . . . . . 5

1.3 Schematic drawing of a zone plate . . . . . . . . . . . 7

1.4 Schematic ray diagram of the BESSY II TXM . . . . . . . . . . 9

1.5 Schematic ray diagram of the STXM . . . . . . . . . . . . . 10

1.6 Variables used for the calculation of the STXM imaging properties 11

1.7 Relative intensity in the zone plate focal plane . . . . . . . . . . . 12

1.8 STXM contrast transfer functions . . . . . . . . . . . . . . . 13

1.9 STXM contrast transfer functions for three different central stops diameters . . . . . . . . . . . . . . . . . 14

1.10 Influence of positioning noise on the contrast transfer . . . . . . 16

1.11 Number of incident photons per pixel required as a function of specimen spatial frequency . . . . . . . . . . . . . . . 19

2.1 Schematic overview of the scanning transmission X-ray microscope at BESSY II . . . . . . . . . . . . . . . . . . . . . . . . 22

2.2 Brilliance of the undulator U41 . . . . . . . . . . . . . . 22

2.3 Photograph of the scanning transmission X-ray microscope . . . . 23

3.1 Spectrum of the undulator U41 at the zone plate . . . . . . . . 28

3.2 Spatial distribution of the direct beam of the first and second harmonic of the undulator U41 . . . . . . . . . . . . . . . 29

3.3 Total power emanating from the undulator U41 . . . . . . . 30

3.4 Source size and divergence of the undulator U41 . . . . . . . . 30

3.5 Schematic monochromator principle . . . . . . . . . . . 32

3.6 Measured diffraction efficiency of the monochromator grating . . . 33

3.7 Calculated monochromaticity of the STXM monochromator . . . 34

3.8 Reflectivity of the different mirror coatings . . . . . . . . . . 36

3.9 Floor plan of the U41 beamlines . . . . . . . . . . . . . . . . 37

3.10 Schematic diagram of the STXM beamline . . . . . . . . . 37

3.11 Drawing of the STXM monochromator . . . . . . . . . . . . . 39

3.12 The principle of the mirror translation of the monochromator . . 41 
3.13 Deviation from the center of the beam impinging on the grating .

3.14 Photon rate in the water window energy range measured at the beam monitor . . . . . . . . . . . . . . . . .

3.15 Photon rate in the focal spot at the carbon absorption edge measured with the pn-CCD detector . . . . . . . . . . . 46

3.16 NEXAFS spectrum of $\mathrm{CO}_{2}$ at the carbon $K$ absorption edge . . . 47

3.17 Measurement of the second order radiation . . . . . . . . . . . . . 49

4.1 Schematic STXM ray diagram for first and third order imaging . . 52

4.2 Maximum image diameter for several OSA diameters . . . . . . 53

4.3 The STXM zone plates . . . . . . . . . . . . . . 54

4.4 Nanostructuring of STXM zone plates . . . . . . . . . . 55

4.5 Cut-away view of the object stage . . . . . . . . . . . 56

4.6 Cut-away view of the in-vacuum zone plate $\mathrm{Z}$ drive . . . . . . . . 58

4.7 Test setup for measuring the accuracy of the piezo stage motion . 61

4.8 Repeatability of the piezo stage . . . . . . . . . . . . . 61

4.9 Nonlinearity of the piezo stage . . . . . . . . . . . . . . . . 62

4.10 Exploded view of the wet specimen chamber . . . . . . . . 63

5.1 Operating principle of the charge coupled device (CCD) . . . . . 66

5.2 Schematic cross section of the pn-CCD . . . . . . . . . . . . 69

5.3 Operating principle of the pn-CCD . . . . . . . . . . 70

5.4 Illumination of the pn-CCD at three different exposure times . . . 71

5.5 The detector vacuum vessel . . . . . . . . . . . . . 72

5.6 Schematic overview of the pn-CCD electronic components . . . . 73

5.7 Screen shot of the sequencer setup program . . . . . . . . . . . 74

5.8 Relative amplification of the 64 channels of the STXM pn-CCD. . 76

5.9 Charge transfer efficiency of the 64 channels of the STXM pn-CCD. 77

5.10 Noise level of the STXM pn-CCD detector . . . . . . . . . . . . 77

5.11 Noise level histogram of the STXM pn-CCD detector . . . . . . . 78

5.12 Measured quantum detection efficiency of a pn-CCD detector . . . 79

5.13 Test of the pn-CCD Reset FET mechanism . . . . . . . . . . 80

5.14 Linearity of the pn-CCD detector . . . . . . . . . . . . 81

6.1 DC motor scan of chernozem dry sample on gold grid . . . . . . 84

6.2 Piezo scan image without specimen . . . . . . . . . . . . . . . 84

6.3 Piezo scan image of diatoms . . . . . . . . . . . . . . . . 85

6.4 Simulated knife edge scan for three central stop diameters . . . . 86

6.5 Piezo scan image of gold spheres as resolution test object . . . . . 87

6.6 Power spectrum of gold sphere test object . . . . . . . . . . . 88

6.7 Focus scan with astigmatic zone plate . . . . . . . . . . . . . . 88

6.8 Elemental imaging below and above an absorption edge . . . . . . 89

6.9 Carbon mass density of a chernozem flocks. . . . . . . . . . . . 90

6.10 Differential phase contrast image of chernozem particles . . . . . . 91 
6.11 Amplitude and differential phase contrast image of two diatoms . 92

A.1 Overview of the STXM control computers . . . . . . . . . . 97

A.2 Schematic diagram of the monochromator PID control . . . . . 101

B.1 The zone plate holder . . . . . . . . . . . . . . . . . . . 104

B.2 Silicon vacuum window on holder . . . . . . . . . . 105

B.3 Cut-away view of the OSA . . . . . . . . . . . . 105

B.4 The detector vacuum window holder . . . . . . . . . 106 



\section{List of Tables}

3.1 Characteristics of the BESSY II storage ring and the undulator U41 27

3.2 Source diameter and divergence of the undulator U41 . . . . . . 28

3.3 Properties of the gratings employed in the STXM monochromator 34

3.4 The position of the mirror axis of the monochromator with the parameters used for the calculation. . . . . . . . . . . . 42

4.1 Parameters of the currently used STXM zone plates for the STXM 54

4.2 Operation of the object stage movements . . . . . . . . . . 57

6.1 Theoretical knife edge resolution for zone plates with and without central stop . . . . . . . . . . . . . . . . . 86

A.1 The main STXM user programs . . . . . . . . . . . . . . . . 99

A.2 The Real Time Linux kernel modules for control of the object stage and of the monochromator . . . . . . . . . . . . 100 



\section{Introduction}

Many areas of scientific investigation require chemical analysis with sub-micron spatial resolution. Established chemically sensitive methods such as infrared spectroscopy or nuclear magnetic resonance spectroscopy do not have very high spatial resolution. Electron microscopy has excellent spatial resolution but provides only elemental, but no chemical sensitivity. Furthermore, its use is often prohibited for radiation sensitive samples by the strong radiation damages induced by the electrons. X-ray spectromicroscopy is a method that combines the sub- $100 \mathrm{~nm}$ spatial resolution of X-ray microscopes with the ability to distinguish different chemical states via NEXAFS spectroscopy [1].

In the soft $\mathrm{X}$-ray energy range between the carbon and oxygen $\mathrm{K}$ absorption edges at $283 \mathrm{eV}$ and $543 \mathrm{eV}$ (corresponding to a wavelength of $4.4 \mathrm{~nm}$ and $2.3 \mathrm{~nm}$, respectively), the so-called water window [100], specimens in water show a natural absorption contrast, which permits imaging of unstained specimens in their natural hydrated state. Samples of about $10 \mu \mathrm{m}$ thickness can be imaged as a whole [71,40]. Using zone plates as X-ray objectives [70], spatial resolutions in the $20 \mathrm{~nm}$ to $100 \mathrm{~nm}$ range are obtained [27].

Close to absorption edges, X-ray absorption spectra show resonances generated by the transition of the excited photoelectrons to unoccupied molecular orbitals or continuum states. This near edge absorption fine structure (NEXAFS) is characteristic for the different chemical states [78]. In combination with X-ray microscopy, NEXAFS is used to generate chemical maps of specimens with high spatial resolution [36].

There are two kinds of X-ray microscopes: transmission X-ray microscopes (TXMs) image the sample onto a spatially resolving detector; scanning transmission X-ray microscopes (STXMs) scan the sample with an X-ray microprobe and record the transmitted intensity for each scan pixel. STXMs deliver a dose to the sample which is typically one order of magnitude lower compared to TXMs [40]. This is important especially for spectromicroscopy of radiation sensitive samples, since breaking of chemical bonds cannot be prevented by cryofixation [8], which is used in TXMs to prevent structural changes in the specimen [68]. While the spatial resolution of STXMs is typically slightly lower than that of TXMs due to technical reasons, monochromators with high spectral resolution can be built with a much simpler optical setup than for a TXM. Therefore, STXMs are very well suited for spectromicroscopy of radiation-sensitive samples. Furthermore, 
STXMs can image specimens in advanced contrast modes such as differential phase contrast or dark field contrast without additional optics [47]. Acquiring images in phase contrast mode is advantageous because of the higher contrast achievable, particularly at higher photon energies. In addition, one can choose a photon energy with high phase contrast but low absorption to minimize the radiation damage to the sample. Dark field contrast is useful to image specifically labeled specimens [86].

The usefulness of a STXM for spectromicroscopy research is determined not only by the spectral and spatial resolution but also by a number of more practical issues: The photon rate in the focal spot has to be high to obtain short image and spectrum acquisition times. The beamline and the microscope should be easy to align. The energy set with the monochromator has to be reproducible if spectra of different specimens are to be compared. If a large number of specimens is studied, they have to be exchanged rapidly. Finally, the detector must have a linear response up to very high photon rates and the noise of the measured intensity should be limited by the photon noise, not the detector readout noise.

The above requirements are addressed in the development of the new STXM and its monochromator at the BESSY II storage ring, which are described in this thesis. In the first chapter, the interaction of X-rays with matter and the image formation in the STXM are discussed. The obtained contrast transfer functions for the STXM are used to calculate the minimum photon numbers and the required accuracy of the scanning movement to obtain images of good quality. Chapter 2 gives a short overview of existing X-ray microscopes and of the new STXM at BESSY II. The next chapters follow the beam path from the monochromator via the object stage to the STXM detector. Finally, first images acquired with the STXM in amplitude contrast, differential phase contrast and elemental contrast are presented in chapter 6 .

Due to strong interest of the scientific community in the fields of environmental sciences in Germany, the Federal Ministry for Science and Education (BMBF) has funded this development. Division 411 (Basic Scientific Research) has covered the costs for beamline and personnel, whereas division 421 (Social-Ecological Environmental Research), has funded this newly developed STXM. 


\section{Chapter 1}

\section{X-Ray Microscopy}

\subsection{Interaction of Soft X-rays With Matter}

In this section, a short introduction to the interaction of soft X-rays with matter is given. A comprehensive description may be found e.g. in Ref. 5. Soft X-rays interact with matter solely by either photoelectric absorption or elastic scattering. Inelastic or Compton scattering occurs only for multi-keV X-radiation. The cross section for elastic scattering is much lower than the absorption cross section, so multiple elastic scattering may be neglected. Furthermore, there are no strong variations of the refraction index at specimen discontinuities, so in contrast to visible light microscopy, clear images can be obtained even from thick specimens.

The absorption and phase shift of X-rays traveling in a homogeneous material can be described macroscopically by the energy-dependent complex refractive index $n$, which is commonly written as:

$$
n=1-\delta-i \beta .
$$

It can also be expressed in microscopic terms, namely the real and imaginary part $f_{1}^{0}$ and $f_{2}^{0}$ of the complex atomic scattering factor for forward scattering [5]:

$$
n=1-\frac{\mathrm{n}_{\mathrm{a}} \mathrm{r}_{\mathrm{e}} \lambda^{2}}{2 \pi}\left(f_{1}^{0}+i f_{2}^{0}\right)
$$

where $n_{\mathrm{a}}$ is the atomic density of the material, $\mathrm{r}_{\mathrm{e}}$ is the classical electron radius, and $\lambda$ is the X-ray wavelength. $f_{1}^{0}$ and $f_{2}^{0}$ are tabulated for all relevant elements in the soft X-ray energy range [31].

A plane wave traveling in vacuum with wavenumber $k=2 \pi / \lambda$ and circular frequency $\omega$ can be written as

$$
\psi_{0}(z)=A \mathrm{e}^{i(\omega t-k z)} .
$$

If the wave propagates in a homogeneous material, the vacuum wave number has to be multiplied by the complex refractive index $n$ :

$$
\psi(z)=A \mathrm{e}^{i(\omega t-n k z)}=\underbrace{A \mathrm{e}^{i(\omega t-k z)}}_{\text {vacuum propagation }} \times \underbrace{\mathrm{e}^{i \delta k z}}_{\text {phase shift }} \times \underbrace{\mathrm{e}^{-\beta k z}}_{\text {absorption }} .
$$




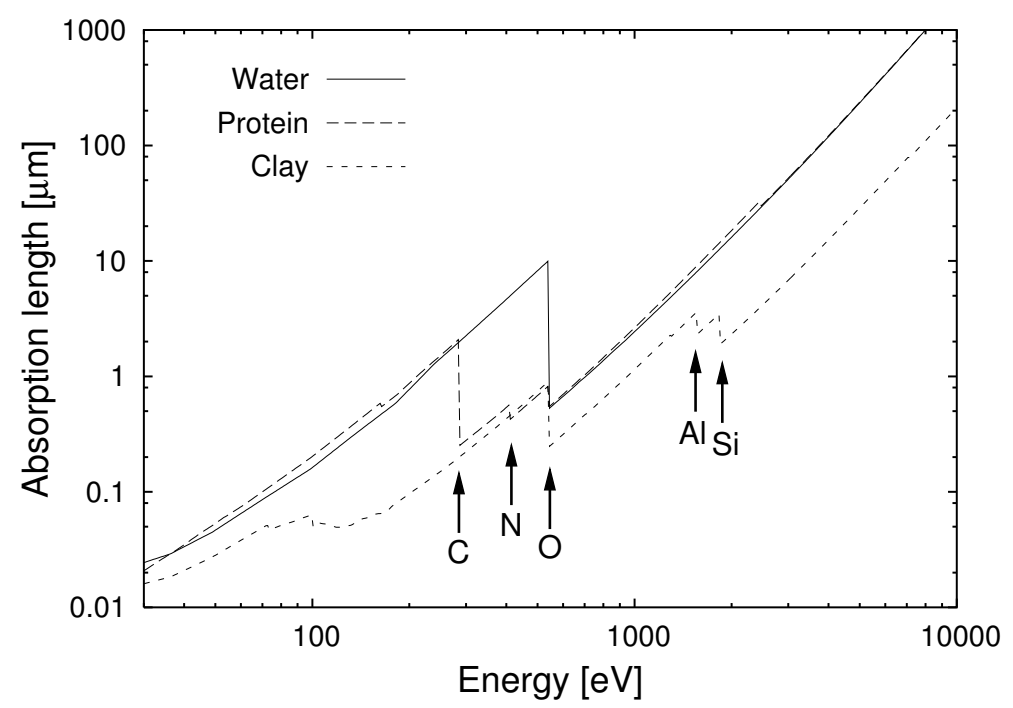

Figure 1.1: 1 /e absorption length $\mu_{1}^{-1}$ of water and protein and clay as examples for biology and soil science specimens. $\mathrm{C}_{94} \mathrm{H}_{139} \mathrm{~N}_{24} \mathrm{O}_{31} \mathrm{~S}$ with a density of $\rho=1.35 \mathrm{~g} / \mathrm{cm}^{3}$ is shown as a model for protein, montmorillonite $\left(\mathrm{Al}_{1.77} \mathrm{Fe}_{0.03} \mathrm{Mg}_{0.2} \mathrm{Si}_{3.74} \mathrm{Al}_{0.26} \mathrm{O}_{10}(\mathrm{OH})_{2}\right.$, $\rho=2.75 \mathrm{~g} / \mathrm{cm}^{3}$ ) [80] as an example for a clay mineral. Some of the K shell absorption edges (see Sec. 1.1.1) are indicated with their corresponding elements. Data from Ref. 31.

Hence, $\delta$ gives the phase shift of the material and $\beta$ the absorption.

The intensity absorption is also commonly expressed by the linear absorption coefficient

$$
\mu_{1}=2 \beta k=\frac{4 \pi \beta}{\lambda}
$$

or the mass attenuation coefficient

$$
\mu_{\mathrm{m}}=\frac{\mu_{1}}{\rho}
$$

where $\rho$ is the mass density of the material. The inverse of the linear absorption coefficient gives the absorption length $\mu_{1}^{-1}$ of the material, after which the intensity $\psi^{2}(z)$ of the wave drops by a factor of e.

Fig. 1.1 shows the absorption length for water, a model protein and a specific clay as examples for biology and soil science specimens. In the energy range between the $\mathrm{K}$ absorption edges (see Sec. 1.1.1) of carbon $(283 \mathrm{eV})$ and oxygen $(543 \mathrm{eV})$, water is one order of magnitude more transparent than both protein and clay. This energy range, the so called water window, has been proposed in 1951 by Wolter for X-ray microscopy of hydrated biological samples [100]. At the low-energy side of the oxygen absorption edge, hydrated specimens can be imaged in a water layer of up to about $10 \mu \mathrm{m}$ thickness with high absorption contrast. Because of the high natural absorption contrast, specimens can be imaged in their natural state without staining. 


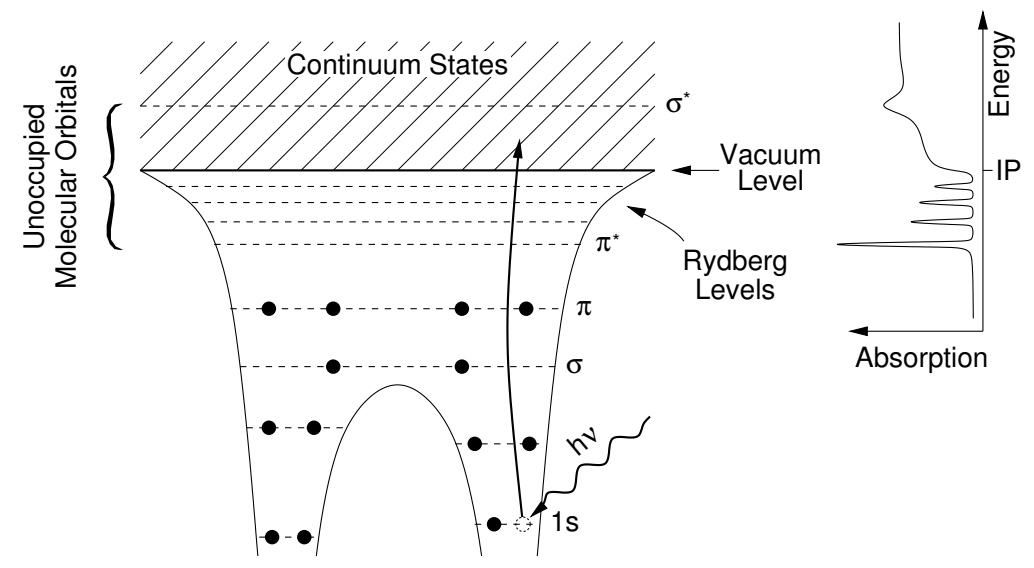

Figure 1.2: Schematic electron potential (left) and K-shell absorption spectrum (right) of a diatomic molecule. The electron transition from the $1 \mathrm{~s}$ level to the unoccupied orbitals caused by the absorption of a photon gives rise to absorption resonances at the corresponding energy levels below and above the ionization potential (IP). For photon energies above the IP, the photoelectron receives a kinetic energy of the difference between the photon energy and the IP. Therefore, the absorption fine structure is also influenced by electron scattering processes (see text).

\subsubsection{X-ray Spectroscopy for Elemental and Chemical Map- ping}

If the photon energy reaches the threshold required to excite electrons from an inner shell, the absorption rises rapidly. This causes absorption edges in the spectrum which are characteristic for the different shells of each element (see Fig. 1.1). If a specimen is imaged both below and above an absorption edge, the quotient image shows the distribution of the corresponding element. With the absorption data for the element [31], one can calculate a quantitative map of the area mass density of the specimen for the selected element.

With sufficient monochromaticity, fine structure can be observed in spectra near absorption edges, the so-called near edge absorption fine structure (NEXAFS), also called X-ray absorption near-edge structure (XANES). A detailed discussion of NEXAFS may be found in Ref. 78 .

Fig. 1.2 illustrates the photon absorption process in a diatomic molecule. Two different mechanisms lead to fine structure at absorption edges:

1. Transitions from the ground state to unoccupied molecular orbitals give rise to strong absorption resonances.

2. If the photon energy is above the ionization potential, a photoelectron is emitted from the atom. Depending on the de Broglie wavelength of the photoelectron, it interferes either constructively or destructively with the waves 
backscattered by the neighboring atoms. This causes weaker oscillations of the photon absorption.

The terms NEXAFS or XANES are used for the fine structure generated by the electron transition to unoccupied molecular orbitals and to lower-energetic continuum states (up to $10-40 \mathrm{eV}$ above the ionization potential), where the photoelectron suffers multiple scattering. The absorption modulation caused by higher energy photoelectrons (above about $40 \mathrm{eV}$ kinetic energy), where single scattering at the neighboring atoms predominates, is called extended X-ray absorption fine structure (EXAFS) [4]. An example NEXAFS spectrum is shown in Fig. 3.16.

Because the electronic configuration is characteristic for each chemical bond, it is possible to distinguish different chemical states of a single element based on their NEXAFS spectra. If images are acquired at a set of different energies where the specimen constituents have prominent NEXAFS features, density maps of the different substances can be calculated if the reference spectra of the pure constituents are known.

It is also advantageous to record image sequences, so-called stacks, at closely spaced photon energies around an absorption edge [36]. The images are aligned after the measurement to correct lateral deviations due the focusing movement. From the resulting data set, spectra of arbitrary specimen regions or of single pixels corresponding to sub-100 $\mathrm{nm}$ spots can be obtained. With the linear algebra method of principal component analysis, regions of the specimen with common spectral features may be identified from a stack data set without prior knowledge of their NEXAFS spectra [37].

\subsection{Transmission Zone Plates as High Resolu- tion X-Ray Optics}

In microscopy, the obtainable spatial resolution is limited by the light wavelength and by the objective numerical aperture. In visible light microscopy, the maximum numerical aperture can be reached, so the wavelength determines the spatial resolution. X-ray objectives are still far away from the maximum numerical aperture, so the objective aperture determines the resolution. Therefore, high resolution optics are the key component for X-ray microscopy.

Because of the lack of transparent and sufficiently phase shifting materials in the soft X-ray region below $10 \mathrm{keV}$, refractive lenses cannot be employed for soft $\mathrm{X}$-ray microscopy. Reflective optics as used in X-ray astronomy cannot be used for high-resolution X-ray objectives because of the extremely high requirements concerning the roughness and the accuracy of mirror surfaces.

Modern high-resolution X-ray microscopes use zone plates as focusing elements. Zone plates are circular diffraction gratings with radially increasing line density. In their simplest form, they consist of $N$ alternating opaque and transparent zones (see Fig. 1.3), where the zones are located such that the path lengths 

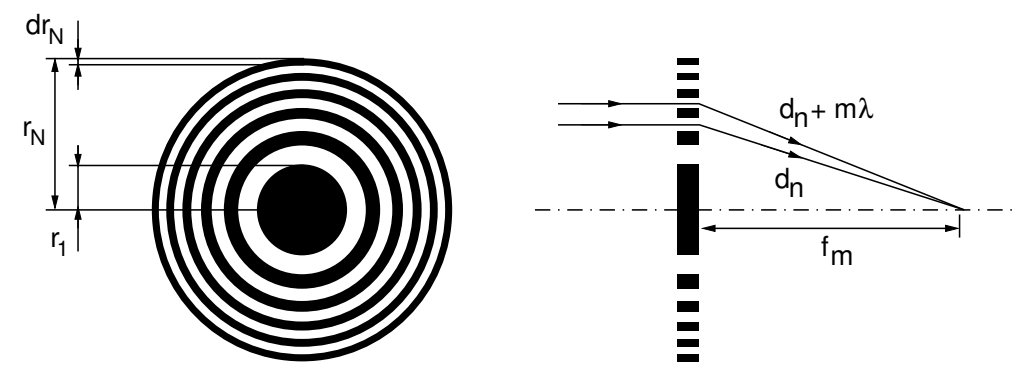

Figure 1.3: Schematic drawing of a zone plate with $N$ concentric zones with radii $r_{n}$. Left: view along the optical axis. Right: cross section. If the zone plate is illuminated with parallel light, the rays from the transparent zones interfere constructively if the optical paths from any two adjacent transparent zones to the $m^{\text {th }}$ diffraction order focal spot $f_{m}$ differ by $m$ wavelengths $\lambda$.

through adjacent transparent zones differ by $m$ wavelengths $\lambda$ and the rays from the different zones interfere constructively in the $m^{\text {th }}$ diffraction order focal spot $f_{m}$. To fulfill this condition, the radius $r_{n}$ of the $n^{\text {th }}$ zone has to be [5]

$$
r_{n}^{2}=m n \lambda f_{m}+\frac{m^{2} n^{2} \lambda^{2}}{4}, \quad n=1 \ldots N
$$

For large focal lengths $\left(f_{m} \gg m n \lambda / 4\right)$, the second term, which corrects the spherical aberration if a source at an infinite distance from the zone plate is imaged, can be ignored, so the focal length is

$$
f_{m}=\frac{r_{N}^{2}}{m N \lambda}
$$

The first derivative of Eq. 1.8 with respect to $\mathrm{N}$ gives the outermost zone width $\mathrm{d} r_{N}$ :

$$
\mathrm{d} r_{N}=\frac{r_{N}}{2 N}
$$

For a given diffraction order, the imaging properties of zone plates are equivalent to those of thin lenses [69], for which the Rayleigh criterion gives a maximum transverse resolution in case of monochromatic illumination and incoherent imaging conditions (see Sec. 1.4) of

$$
\delta_{\mathrm{t}}=0.61 \frac{\lambda}{N \cdot A}
$$

with the numerical aperture N.A.. For zone plates with small numerical apertures, the Rayleigh resolution is [5]

$$
\delta_{\mathrm{t}}=1.22 \frac{\mathrm{d} r_{N}}{m}
$$


Therefore, the resolution obtainable with a zone plate in the first diffraction order, which is most commonly used for imaging, is approximately given by the outermost zone width. By imaging in higher diffraction orders $m$, a higher resolution can be achieved.

For thin zone plates with evenly spaced opaque and transparent zones, scalar theory gives a theoretical diffraction efficiency in uneven diffraction orders of

$$
\eta_{m}=1 / m^{2} \pi^{2}
$$

no radiation is diffracted into even diffraction orders [5]. Therefore, the first order diffraction efficiency is about $10 \%$. By replacing the opaque zones with material shifting the phase by $\pi$, the efficiency can be increased.

Diffraction efficiency calculations with coupled-wave theory taking into account the three-dimensional wave propagation in the zone plate show that the higher order efficiency can be increased dramatically for certain high aspect ratios of the zones $[67,28]$. This makes imaging with both high diffraction efficiency and very high resolution possible.

The nanofabrication of zone plates with small outermost zone widths for highresolution microscopy is still technologically challenging. Currently, zone plates with $\mathrm{d} r_{n}=20 \mathrm{~nm}$ can be manufactured with efficiencies close to the theoretical value [57].

Since the zone plate focal length is inversely proportional to the wavelength (see Eq. (1.8)), zone plates show strong chromatic aberrations. To avoid a degradation of the imaging properties, zone plates have to be illuminated with radiation with a monochromaticity $\lambda / \Delta \lambda$ equal to the number of zones times the diffraction order [5]:

$$
\frac{\lambda}{\Delta \lambda} \geq m N
$$

\subsection{Transmission X-Ray Microscopes and Scanning Transmission X-Ray Microscopes}

Similarly to visible light and electron microscopes, two basic types of X-ray microscopes with zone plate optics can be distinguished, the transmission X-ray microscope (TXM) and the scanning transmission X-ray microscope (STXM).

In a TXM, the sample is illuminated by a condenser and imaged with an objective zone plate onto a spatially resolving detector with a typical magnification of order 1000. Fig. 1.4 shows the schematic setup of the BESSY II TXM with the rotating condenser-monochromator [54,27]. An off-axis transmission zone plate acts as the dispersing element. Rotating mirrors are used to generate a hollow cone illumination with a numerical aperture matched to objective zone plates of arbitrary outermost zone width and with a monochromaticity of up to several thousands. With a phase-shifting ring in the back focal plane of the objective 
zone plate and with an annular condenser aperture, Zernike phase contrast can be realized $[73,74]$.

Fig. 1.5 shows the schematic setup of the scanning transmission X-ray microscope. In the STXM, the monochromatic beam is focused by the zone plate and forms a microprobe which is used to scan the sample by moving either the sample or the zone plate. The transmitted intensity is measured with a fast detector. A central stop on the zone plate and a so-called order sorting aperture (OSA) prevent light of diffraction orders other than the one used for imaging from reaching the sample.

Because there are no optical components between the specimen and the STXM detector, the dose delivered to the specimen in amplitude contrast is typically one order of magnitude lower than in the TXM. This is important especially for spectromicroscopy of radiation sensitive samples, since the breaking of chemical bonds cannot be prevented by cryofixation [8].

Most STXMs employ a detector without spatial resolution which integrates the transmitted light. Using a spatially resolving detector, images in various contrast modes like dark field or phase contrast can be obtained without additional optics (see Sec. 1.4). If the fluorescence X-rays excited by the focused primary beam are detected using an energy-resolving detector, the spatial distribution of several elements can be obtained simultaneously in a single image scan. Fluorescence imaging is interesting especially for imaging with multi-keV radiation, where maps of elements of biological relevance like phosphorus, sulphur, potassium, and calcium can be obtained.

In both the STXM and the TXM, imaging in differential interference contrast is possible with a zone plate doublet or with the central stop placed far in front of the STXM zone plate $[98,41]$.

In a STXM, images of arbitrary dimensions can be acquired in contrast to the TXM, where the condenser typically illuminates an image field of 10-20 $\mathrm{mm}$ and larger images can only be obtained by stitching multiple images together in the computer. To obtain diffraction-limited resolution, the STXM zone plate has

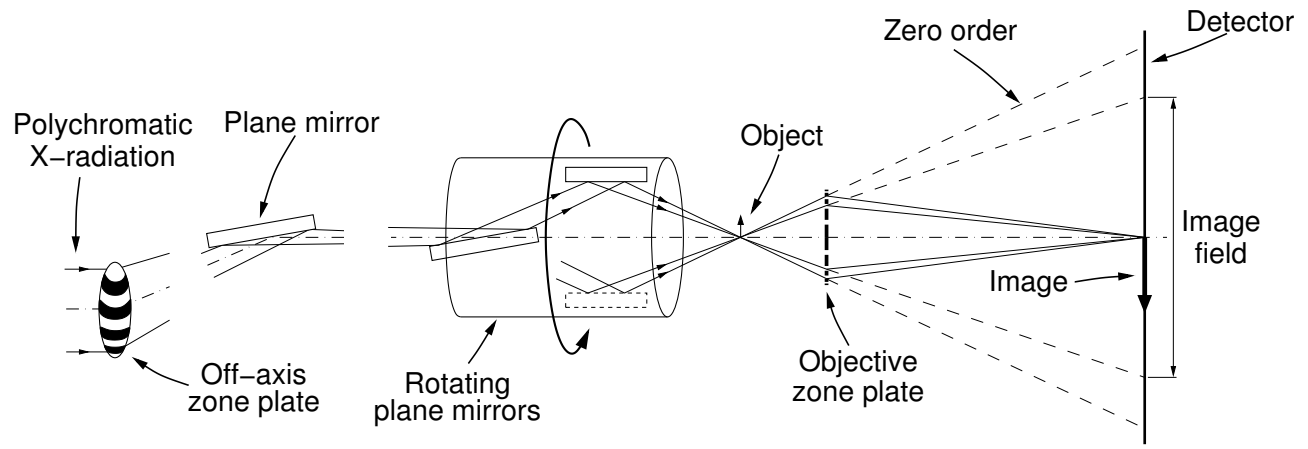

Figure 1.4: Schematic ray diagram of the BESSY II transmission X-ray microscope (TXM) with the rotating condenser-monochromator. 


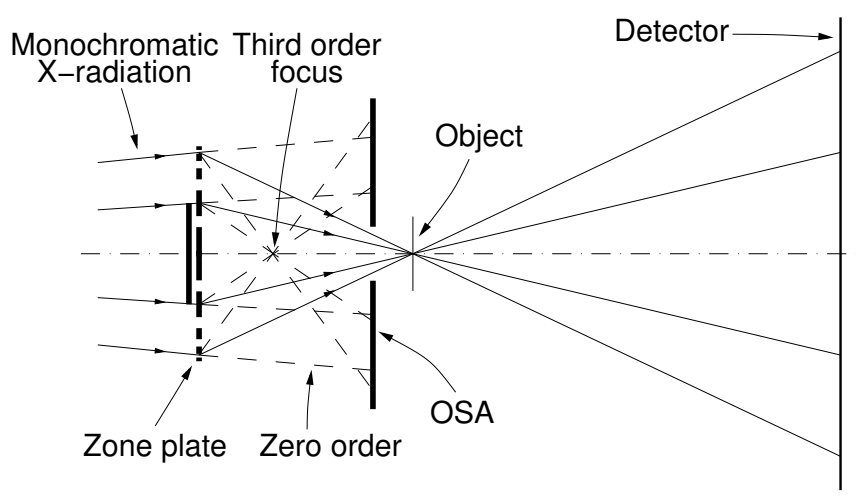

Figure 1.5: Schematic ray diagram of the scanning transmission X-ray microscope (STXM) operating in the first diffraction order of the zone plate. For the image scan, either the zone plate or the object is moved and the transmitted intensity is measured with the detector.

to be illuminated with spatially coherent radiation (see Sec. 3.2.2). Therefore, most STXMs are operated at undulator beamlines (see Sec. 3.1). TXMs are most commonly used with incoherent illumination. Since only the spatially coherent part of the beam may be used, the typical STXM image acquisition time is of order minutes, whereas the TXM exposure time is of order seconds. The influence of problems such as beam instability, vibrations and thermal drift increases with exposure time, so in practice, TXM images have better spatial resolution.

\subsection{Image Formation in the STXM}

In this section, a short overview of the image formation in the scanning transmission X-ray microscope using Fourier optics [23] is given with example calculations for the BESSY II STXM. Detailed discussions may be found e.g. in Refs. 19 and 87.

The variables used for the calculation of the optical properties are shown in Fig. 1.6. The wavefield in the zone plate focal plane is described by the complex probe function $\mathrm{p}(\vec{r})$. Because the refraction index of all materials is very close to unity for X-rays, multiple scattering in the specimen may be neglected. This is called the first Born approximation [9]. If the specimen is thin, it can be described by a two-dimensional transmission function $\mathrm{h}(\vec{r})$. For a homogeneous specimen with the local thickness $\mathrm{t}(\vec{r})$, the complex amplitude transmission (see Eq. (1.4)) is given by

$$
\mathrm{h}(\vec{r})=\exp \left[\frac{2 \pi}{\lambda}(i \delta \mathrm{t}(\vec{r})-\beta \mathrm{t}(\vec{r}))\right] .
$$

For this discussion, it is assumed that the specimen is scanned for the image acquisition with the specimen displacement $\vec{r}_{0}$. The complex wavefield exiting 


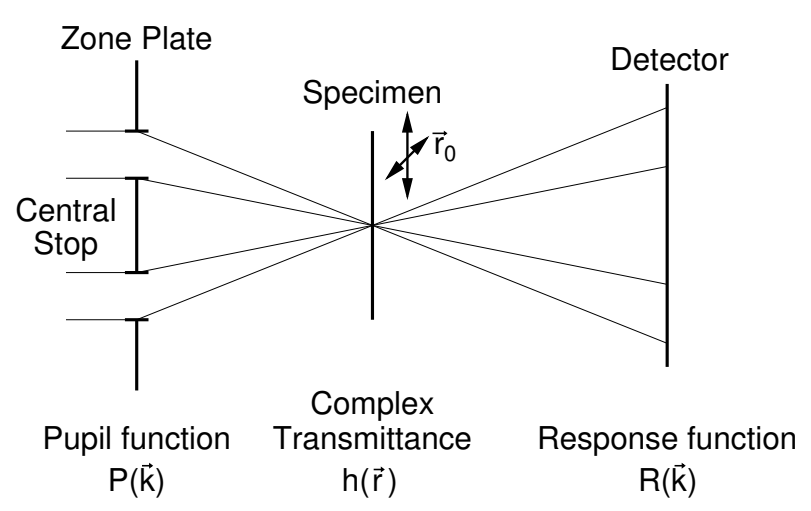

Figure 1.6: Illustration on the variables used for the calculation of the STXM imaging properties. The two-dimensional specimen with complex amplitude transmission $\mathrm{h}(\vec{r})$ is illuminated with the pupil function $\mathrm{P}(\vec{k})$, which is determined by the annular aperture created by the zone plate with the central stop. For each position $\vec{r}_{0}$ of the specimen during the image scan, the transmitted radiation is registered by the detector with the response function $\mathrm{R}(\vec{k})$. Positions in the sample plane are given by vector $\vec{r}$, while the pupil function and the detector response function are given in terms of the spatial frequency $\vec{k}$. The lateral position $\vec{r}_{z}$ at a distance $z$ from the specimen and the spatial frequency $\vec{k}$ are related by the equation $\vec{k}=\vec{r}_{z} /(\lambda z)$ (see Eq. 1.17).

the specimen is

$$
\psi\left(\vec{r}, \vec{r}_{0}\right)=\mathrm{p}(\vec{r}) \mathrm{h}\left(\vec{r}-\vec{r}_{0}\right) .
$$

If the distance $z_{\mathrm{D}}$ of detector from the specimen is large, the Fraunhofer approximation [23] gives a wavefield $\Psi_{0}\left(\vec{r}_{\mathrm{D}}\right)$ in the detector plane of

$$
\Psi_{0}\left(\vec{r}_{\mathrm{D}}\right)=\frac{\exp \left(i k z_{\mathrm{D}}\right) \exp \left[i \frac{k}{2 z_{\mathrm{D}}}\left|\vec{r}_{\mathrm{D}}\right|^{2}\right]}{i \lambda z_{\mathrm{D}}} \iint \psi\left(\vec{r}_{\mathrm{D}}^{\prime}\right) \exp \left[-i \frac{2 \pi}{\lambda z_{\mathrm{D}}} \vec{r}_{\mathrm{D}} \vec{r}_{\mathrm{D}}^{\prime}\right] \mathrm{d}^{2} r_{\mathrm{D}}^{\prime} .
$$

The detector is sensitive only to the intensity, but not to the phase of the incident wave, so the factor preceding the integral gives only a constant factor, which is neglected in the following. Therefore, the wavefield $\Psi$ in the detector plane (neglecting the constant factor) is simply the Fourier transform of the wave in the specimen plane:

$$
\Psi\left(\vec{k}, \vec{r}_{0}\right)=\mathbf{F}_{\vec{r}}\left[\mathrm{p}(\vec{r}) \mathrm{h}\left(\vec{r}-\vec{r}_{0}\right)\right](\vec{r}) \quad \text { with } \quad \vec{k}=\frac{\vec{r}_{\mathrm{D}}}{\lambda z_{\mathrm{D}}}
$$

Likewise, the pupil function $\mathrm{P}(\vec{k})$ is the Fourier transform of the probe function: $\mathrm{P}(\vec{k})=\mathbf{F}_{\vec{r}}[\mathrm{p}(\vec{r})](\vec{r})$. For a zone plate with outermost zone width $\mathrm{d} r_{n}$, the maximum spatial frequency to which the pupil extends in the first diffraction order is

$$
k_{\max }=\frac{r_{N}}{\lambda f_{1}}=\frac{1}{2 \mathrm{~d} r_{N}}
$$




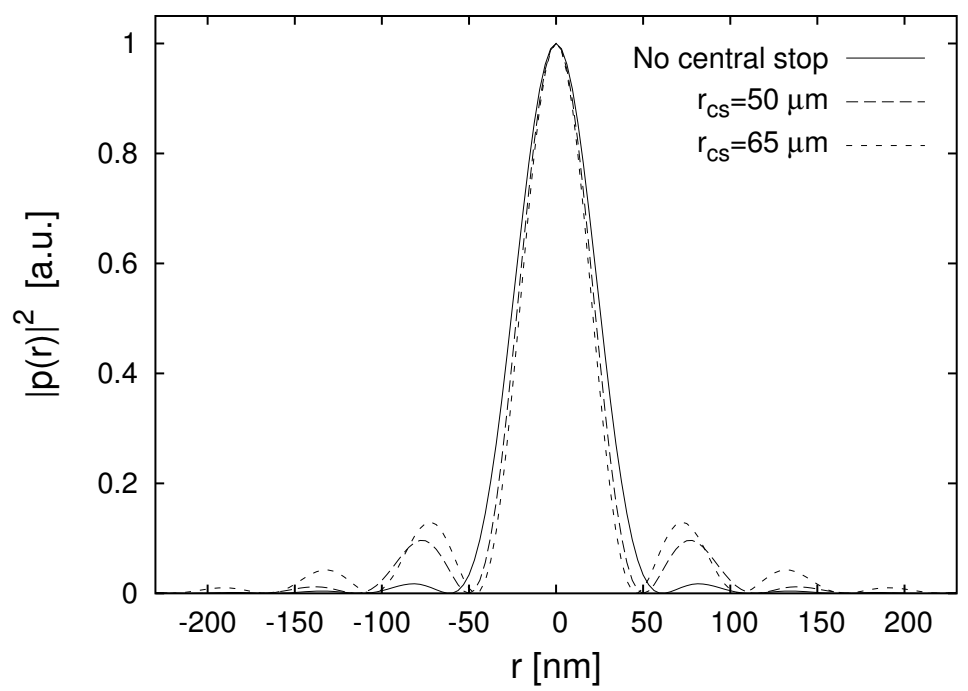

Figure 1.7: Relative intensity $|\mathrm{p}(r)|^{2}$ in the focal plane for the annular aperture generated by a zone plate with central stop. $|\mathrm{p}(r)|^{2}$ is shown for different central stop diameters for a zone plate with outermost zone with $\mathrm{d} r_{N}=50 \mathrm{~nm}$ and radius $r_{N}=100 \mu \mathrm{m}$. The intensities are normalized to a maximum intensity of 1 .

(see Eqs. (1.8) and (1.9)). The modulus squared of $\mathrm{p}(\vec{r})$ is proportional to the intensity in the specimen plane. It is shown in Fig. 1.7 for two different central stop diameters $r_{\mathrm{CS}}$ and without central stop. For a zone plate without central stop, the diffraction pattern is an Airy disk. If the sample is illuminated with a zone plate with a central stop, the central intensity maximum becomes slightly narrower but considerably more intensity is diffracted into the side lobes.

If the detector response function is $\mathrm{R}(\vec{k})$, the image $\mathrm{s}\left(\vec{r}_{0}\right)$ acquired when scanning the specimen is given by

$$
\mathrm{s}\left(\vec{r}_{0}\right)=\iint \mathrm{R}(\vec{k})\left|\Psi\left(\vec{k}, \vec{r}_{0}\right)\right|^{2} \mathrm{~d}^{2} k
$$

For a weakly absorbing and phase shifting specimen, the complex amplitude transmission can be approximated in first order as (see Eq. (1.14)):

$$
\mathrm{h}(\vec{r})=1-\mathrm{a}(\vec{r})-i \phi(\vec{r})
$$

or in reciprocal space

$$
\mathrm{H}(\vec{k})=\delta(\vec{k})-\mathrm{A}(\vec{k})-i \Phi(\vec{k})
$$

Then one can define separate amplitude and phase contrast transfer functions $\mathrm{T}_{\mathrm{a}}(\vec{k})$ and $\mathrm{T}_{\Phi}(\vec{k})$ such that the scan image is calculated simply by a multiplication in reciprocal space:

$$
\mathrm{S}(\vec{k})=-\mathrm{A}(\vec{k}) \mathrm{T}_{\mathrm{a}}(\vec{k})-i \Phi(\vec{k}) \mathrm{T}_{\Phi}(\vec{k})
$$



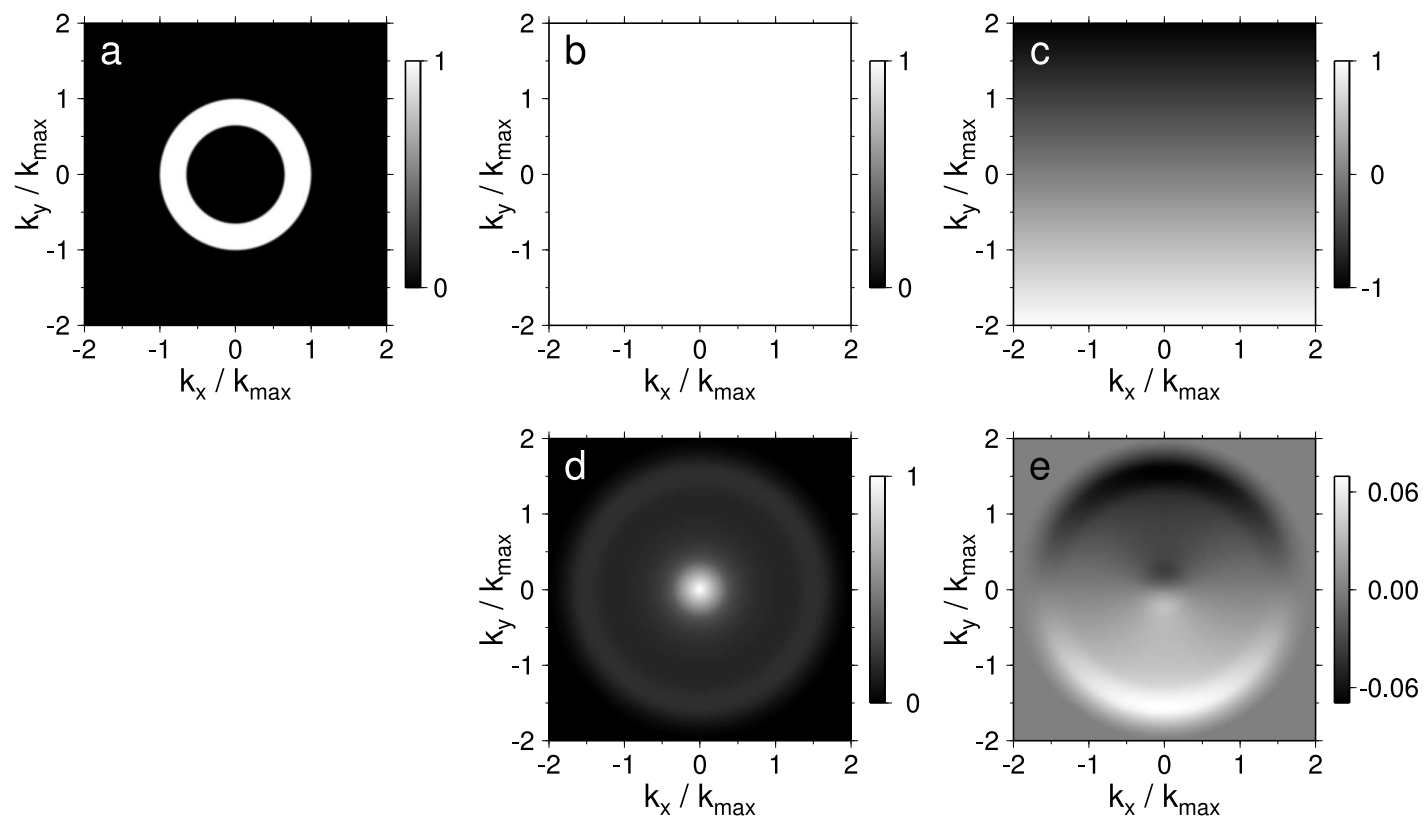

Figure 1.8: Contrast transfer functions as a function of spatial frequency $\vec{k}$ normalized to the maximum pupil frequency $k_{\max }$ (see Eq. 1.18). (a) Pupil function $\mathrm{P}(\vec{k})$ of the zone plate $\left(r_{n}=100 \mu \mathrm{m}\right)$ with central stop $\left(r_{\mathrm{CS}}=65 \mu \mathrm{m}\right)$; (b) constant detector response function $\mathrm{R}(\vec{k})$ corresponding to incoherent bright field imaging; (c) first moment detector response function for differential phase contrast; (d) amplitude contrast transfer function $\mathrm{T}_{\mathrm{a}}(\vec{k})$ for detector response function (b); (e) phase contrast transfer function $\mathrm{T}_{\Phi}(\vec{k})$ for detector response function (c). Not shown are the vanishing phase transfer of response function (b) and amplitude transfer of response function (c). See Fig. 1.9.

The intensity contribution by the zero order pupil is neglected, which causes only an intensity offset in the image. The inverse Fourier transform of $\mathrm{S}(\vec{k})$ gives the real space image:

$$
\mathrm{s}\left(\vec{r}_{0}\right)=\mathbf{F}_{\vec{k}}^{-1}[\mathrm{~S}(\vec{k})]\left(\vec{r}_{0}\right) .
$$

It can be shown [19] that the amplitude and phase contrast transfer functions $\mathrm{T}_{\mathrm{a}}(\vec{k})$ and $\mathrm{T}_{\Phi}(\vec{k})$ for the STXM are

$$
\begin{aligned}
\mathrm{T}_{\mathrm{a}}(\vec{k}) & =\mathrm{C}(0,1, \vec{k})+\mathrm{C}(-1,0, \vec{k}) \\
\mathrm{T}_{\Phi}(\vec{k}) & =\mathrm{C}(0,1, \vec{k})-\mathrm{C}(-1,0, \vec{k}) \\
\text { with } \quad \mathrm{C}(m, n, \vec{k}) & =\iint \mathrm{P}\left(\vec{k}^{\prime}-m \vec{k}\right) \mathrm{P}^{*}\left(\vec{k}^{\prime}-n \vec{k}\right) \mathrm{R}_{k}\left(\vec{k}^{\prime}\right) \mathrm{d}^{2} k^{\prime} .
\end{aligned}
$$

Fig. 1.8 shows the contrast transfer functions for two important detector response functions $\mathrm{R}(\vec{k})$, for a constant detector response and for a detector response proportional to the vertical frequency $k_{\mathrm{y}}$. In Fig. 1.9 the contrast transfer functions 

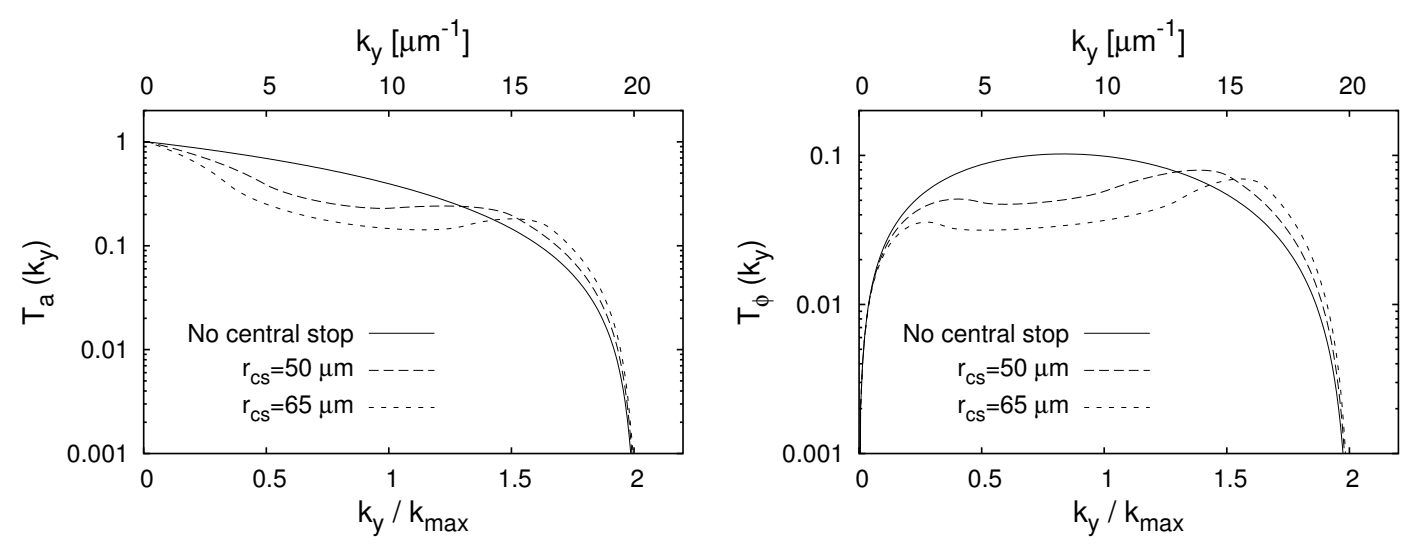

Figure 1.9: Contrast transfer functions as a function of the vertical spatial frequency $k_{y}$ with $k_{x}=0$ (see Fig. 1.8) for two central stop radii $r_{\mathrm{CS}}$ and without central stop. Left: Amplitude contrast transfer $\mathrm{T}_{\mathrm{a}}\left(k_{y}\right)$ for incoherent bright field imaging with the detector response function from Fig. 1.8-b. Right: Phase contrast transfer $\mathrm{T}_{\Phi}\left(k_{y}\right)$ for differential phase contrast imaging with the detector response function from Fig. 1.8-c. The zone plate has a radius of $r_{n}=100 \mathrm{~nm}$ and an outermost zone width of $\mathrm{d} r_{n}=$ $50 \mathrm{~nm}$.

are shown as a function of the vertical spatial frequency $k_{y}$ calculated for two central stop diameters and without central stop.

The constant detector response function leads to an isotropic amplitude impulse transfer up to twice the maximum pupil frequency $k_{\max }$ (Fig. $1.8 \mathrm{~d}$ ) and vanishing phase contrast transfer. Because STXM imaging with a uniform detector response function corresponds to imaging with incoherent illumination in the TXM [76], this contrast mode is called incoherent bright field contrast. When imaging with annular aperture because of the central stop, the contrast at medium spatial frequencies decreases whereas it increases slightly at the highest frequencies (see Fig. 1.9).

If the detector response gives the first moment of the incident intensity (Fig. $1.8 \mathrm{c}$ ), the phase modulation of the specimen is transferred (Fig. $1.8 \mathrm{e}$ ), but the amplitude transfer function vanishes. Unlike the contrast transfer function for the constant detector response, the phase contrast transfer function of the first moment detector is anisotropic and only specimen features with vertical phase modulations are imaged. Specimen modulations with very low spatial frequencies are not transferred, so the absolute phase shift introduced by a specimen cannot be obtained from the image. Therefore, this contrast mode is called differential phase contrast. In Sec. 6.3, example differential phase contrast images are shown.

\subsubsection{Imaging With a Configured Detector}

In the last section it was shown that different contrast modes can be achieved with different detector response functions $\mathrm{R}(\vec{k})$. Therefore, it is desirable to have 
a detector with a set of response functions $\mathrm{R}_{i}$ instead of simply integrating the transmitted intensity. The most flexible approach is the use of a CCD detector for the image registration, where arbitrary response functions can be chosen. Differential phase contrast imaging with this so-called configurable detector has been investigated mainly by Morrison et al. [47, 48, 49]. Dark field contrast imaging with a CCD detector, where only the intensity outside of the zone plate pupil is registered, has been demonstrated by Chapman et al. [13]. Takano et al. showed differential phase contrast images acquired with a configured CCD detector for $8 \mathrm{keV}$-radiation [82].

Because differential phase contrast images generated with the first moment detector show only the local phase gradient, they are difficult to interpret. Therefore, a contrast mode giving the absolute specimen phase shift would be advantageous.

With the method of Wigner distribution deconvolution, the complex specimen transmission can be reconstructed if the two-dimensional diffraction pattern is recorded for each pixel of the raster scan. Such amplitude and phase maps of the specimen have been reconstructed by Chapman et al. using the Stony Brook STXM [14]. In addition to the complex specimen function, the imaging properties of the optical system can be determined. Theoretically, images with a resolution exceeding the limit of $2 k_{\max }$ can be reconstructed from the four-dimensional data set if specimen diffraction orders outside the zeroth order are recorded. However, this so-called super resolution imaging [63] is difficult to realize because of the very low signal diffracted in higher orders for non-periodic specimens.

Feser adapted a method to reconstruct the complex specimen transmission from scanning transmission electron microscopy for the use in a STXM with a segmented detector $[19,20]$. It generates an estimate of the absolute amplitude and phase shift of the specimen by applying a set of filters in Fourier space to the images recorded with the different detector segments.

The estimate $\hat{\mathrm{H}}(\vec{k})$ of the complex specimen transmission $\mathrm{H}(\vec{k})$ is calculated using the ansatz that the specimen estimate can be calculated from the images $\mathrm{S}_{i}$ recorded with $i$-th detector response function as follows:

$$
\hat{\mathrm{H}}=\sum_{k} \mathrm{~W}_{i}(\vec{k}) \mathrm{S}_{i}(\vec{k})
$$

It can be shown that under certain conditions [19] the residual of the specimen estimate is minimized for filter functions

$$
\mathrm{W}_{i}(\vec{k})=\frac{\mathrm{T}_{\mathrm{a}}^{(i) *}(\vec{k})}{\sum_{j}\left|\mathrm{~T}_{\mathrm{a}}^{(j)}(\vec{k})\right|^{2}+\beta_{\mathrm{a}}(\vec{k})}+\frac{\mathrm{T}_{\Phi}^{(i) *}(\vec{k})}{\sum_{j}\left|\mathrm{~T}_{\Phi}^{(j)}(\vec{k})\right|^{2}+\beta_{\Phi}(\vec{k})} .
$$

$\mathrm{T}_{\mathrm{a}}^{(i)}(\vec{k})$ and $\mathrm{T}_{\Phi}^{(i)}(\vec{k})$ are the amplitude and phase contrast transfer functions of the $i^{\text {th }}$ detector element (see Eq. (1.24)). The terms $\beta_{\mathrm{a}}(\vec{k})$ and $\beta_{\phi}(\vec{k})$ may be 


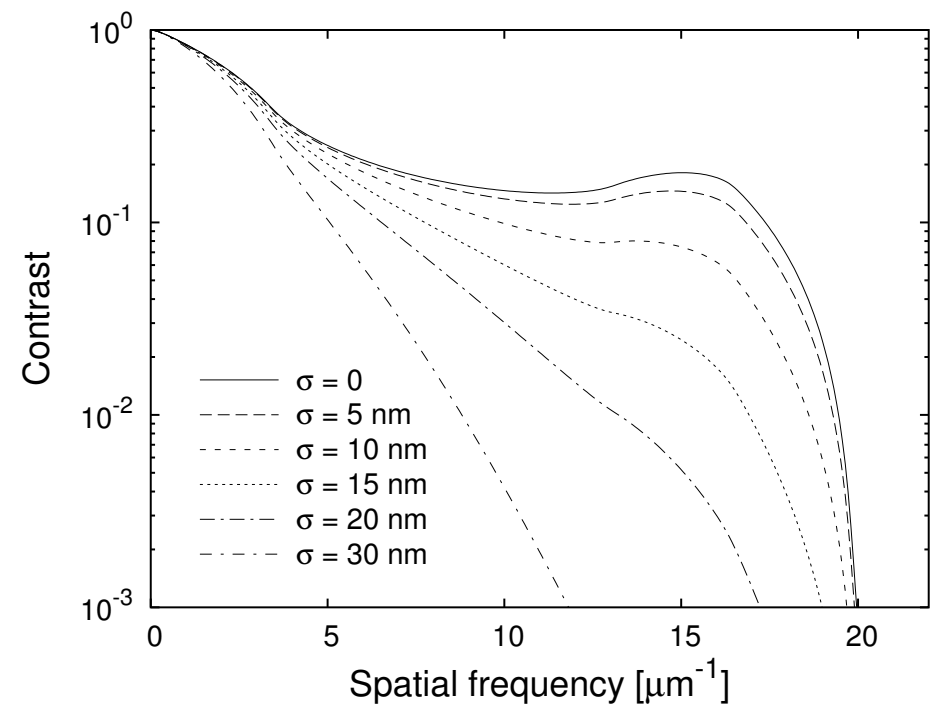

Figure 1.10: Influence of positioning noise on the amplitude contrast transfer for several standard deviations $\sigma$ of the Gaussian position noise distribution. The contrast transfer is calculated for a zone plate with an outermost zone width of $\mathrm{d} r_{N}=50 \mathrm{~nm}$, a radius of $r_{N}=100 \mu \mathrm{m}$, and a central stop radius of $r_{\mathrm{CS}}=65 \mu \mathrm{m}$.

thought of as inverse signal-to-noise ratios of the amplitude and phase part of the image; in practice, they are set to an empirical constant value. Eq. (1.26) resembles a Wiener filter, which is used in digital image processing to deconvolve noisy images [34].

To be able to reconstruct the amplitude and phase of the specimen, it must be precisely in the zone plate focus. If the sample is out of focus, the phase and amplitude part mix and the reconstruction gives incorrect results especially for the phase estimate [19].

With this Fourier filtering method, using either a segmented detector or a CCD detector with a set of different response functions, it is possible to reconstruct a specimen estimate taking into account the contrast transfer functions.

\subsubsection{Contrast and Positioning Noise}

In practice, the contrast obtainable when imaging specimen structures is limited not only by the imaging properties of the optical system but also by the positioning noise of the zone plate focal spot with respect to the specimen. To estimate the degradation of the image by the positioning noise, the amplitude contrast transfer (see Fig. 1.9) is calculated for a Gaussian position error distribution, which is taken into account by multiplying the contrast transfer function with the Fourier transform of the error distribution (see Fig. 1.10). With a standard deviation of the position noise of $\sigma=10 \mathrm{~nm}$, the contrast transfer drops by more than a factor of two at a spatial frequency of $15 \mathrm{\mu m}^{-1}$, whereas the decrease is 
tolerable at $\sigma=5 \mathrm{~nm}$. Therefore, the full width at half maximum of the position noise should not exceed $2.35 \times 5 \mathrm{~nm}=12 \mathrm{~nm}$ to reach the diffraction limited resolution of a zone plate with $50 \mathrm{~nm}$ outermost zone width.

\subsubsection{Signal-to-Noise Ratio and Photon Numbers}

The signal-to-noise ratio is a measure for the degradation of the image quality by the noise in the intensity. In the following, two pixels of the STXM scan image corresponding to specimen areas with transmissions $T_{1}$ and $T_{2}<T_{2}$ are considered. If the sample is illuminated with $N_{0}$ photons per pixel, the mean transmitted photon numbers of the two sample areas are $N_{1}=T_{1} N_{0}$ and $N_{2}=$ $T_{2} N_{0}$. The signal-to noise ratio is the ratio of the difference of the photon numbers and the noise of the difference signal, so for photon numbers obeying a Poisson distribution it can be written as

$$
S N R=\frac{N_{2}-N_{1}}{\sqrt{N_{1}+N_{2}}} .
$$

The Rose criterion gives a minimum signal-to-noise level of three to five for images [64].

Photon detection probabilities $\eta_{\mathrm{D}}$ of the detector less than $100 \%$ and the transmission of the detector vacuum window $T_{\mathrm{Vw}}$ and the sample holder membrane $T_{\mathrm{SH}}$ can be taken into account by multiplying them with the specimen transmissions $T_{1}$ and $T_{2}$ :

$$
T_{1,2}^{\prime}=T_{1,2} * \eta_{\mathrm{D}} * T_{\mathrm{VW}} * T_{\mathrm{SH}}
$$

The contrast is given by

$$
C=\frac{N_{2}-N_{1}}{N_{1}+N_{2}}=\frac{T_{2}-T_{1}}{T_{1}+T_{2}}
$$

The number of photons $N_{0}$ required for the sample illumination to obtain an image of signal-to-noise ratio $S N R$ can be calculated from the contrast and transmission of the specimen as follows:

$$
N_{0}=\frac{1}{2 \bar{T}^{\prime} C^{2}} S N R^{2}
$$

where $\bar{T}^{\prime}=\left(T_{1}^{\prime}+T_{2}^{\prime}\right) / 2$ is the mean transmission including the detection probability and the membrane transmissions. In images with small structures, the contrast depends not only on the transmission of the specimen areas, but also on the contrast transfer function. In the following, $N_{0}$ is calculated for the model system of a sine grating of varying period with a thickness equal to the structure width. The dependence of the contrast transfer on the spatial frequency is taken into account by multiplying the incoherent bright field contrast transfer function 
(see Eq. (1.24)) with the absorption contrast for the photon number calculation. Two specimens are considered as examples for imaging a clay mineral in standard absorption contrast and for differential imaging of an organic coating on a clay layer in elemental contrast at the carbon absorption edge:

Specimen A: montmorillonite sine grating in water imaged in amplitude contrast at $284 \mathrm{eV}$. Montmorillonite is a clay mineral; it can be described as $\mathrm{Al}_{1.77} \mathrm{Fe}_{0.03} \mathrm{Mg}_{0.2} \mathrm{Si}_{3.74} \mathrm{Al}_{0.26} \mathrm{O}_{10}(\mathrm{OH})_{2}$ and has a a typical density of $2.75 \mathrm{~g} / \mathrm{cm}^{3}[80]$.

Specimen B: carbon sine grating on a $500 \mathrm{~nm}$ thick montmorillonite layer in water. The image contrast arises from the different absorption below and above the carbon $\mathrm{K}$ absorption edge.

Both specimens are located in a $1 \mu \mathrm{m}$ thick water layer between the two $100 \mathrm{~nm}$ thick silicon nitride windows of the sample holder. The transmission of a $150 \mathrm{~nm}$ thick detector vacuum window and a detection probability of $50 \%$ are also taken into account for the transmission values. The photon numbers are calculated for a zone plate with an outermost zone width of $\mathrm{d} r_{N}=50 \mathrm{~nm}$, a radius of $r_{N}=100 \mu \mathrm{m}$ and a central stop radius of $65 \mu \mathrm{m}$.

Fig. 1.11 shows the number $N_{0}$ of photons per pixel needed to image the specimens $\mathrm{A}$ and $\mathrm{B}$ with a signal-to-noise ratio of $S N R=3$. To image fine structures with spatial frequencies of $10 \mu^{-1}$ to $15 \mu^{-1}$ corresponding to a structure width of $33 \mathrm{~nm}$ to $50 \mathrm{~nm}, 10^{6}$ photons per pixel are required for specimen A and $2 \times 10^{6}$ photons per pixel for specimen B. If only specimen modulations of $100 \mathrm{~nm}$ width have to be detected, the number of photons can be lowered by an order of magnitude. Specimen structures with medium spatial frequencies could be imaged with fewer photons by using a a zone plate with a smaller central stop, where specimen structures with spatial frequencies of $5-10 \mu^{-1}$ are better transferred (see Fig. 1.9). 


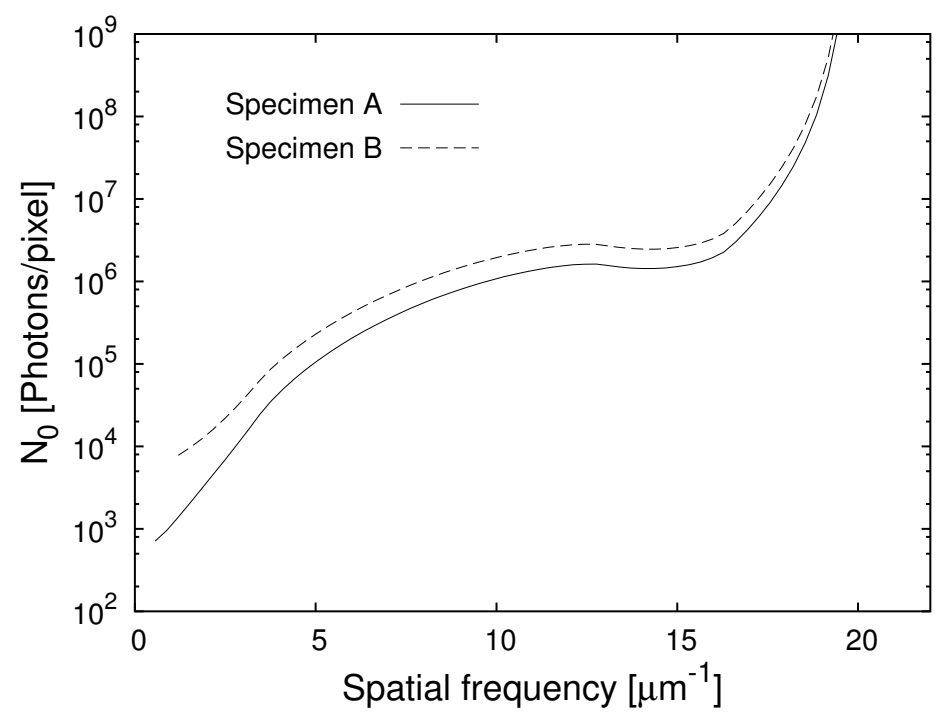

Figure 1.11: Number of photons per pixel in the focal spot $N_{0}$ required for an image with a signal-to-noise ratio of 3 as a function of spatial frequency for two example specimens: a montmorillonite sine grating imaged at $284 \mathrm{eV}$ (specimen A) and a humic acid grating on a $500 \mathrm{~nm}$ thick montmorillonite matrix layer in elemental contrast below and above the carbon absorption edge (specimen B). The photon numbers are calculated for a zone plate with a radius of $r_{\mathrm{n}}=100 \mu \mathrm{m}$, a central stop diameter of $r_{\mathrm{cs}}=65 \mu \mathrm{m}$ and an outermost zone width of $\mathrm{d} r_{\mathrm{n}}=50 \mathrm{~nm}$. 



\section{Chapter 2}

\section{The STXM at the Undulator U41 at BESSY II}

Scanning transmission X-ray microscopy with zone plates has been pioneered by the Stony Brook X-ray microscopy group $[60,33,35]$, which has constructed several STXMs operating at the National Synchrotron Light Source. Currently, two identical room temperature STXMs are located at separate beamline branches. An additional STXM for imaging of frozen hydrated specimens has been built with a provision to acquire specimen tilt-series for computed tomography $[46,88]$.

Two STXMs are operating at an undulator and at a high brilliance bending magnet at the Advanced Light Source with a focus on polymer science [90,2]. To avoid lateral deviations of the focal spot when the zone plate is moved along the optical axis during acquisition of spectrum, the relative position of the zone plate with respect to the specimen is measured with laser interferometers and used for closed loop control [39].

Scanning microscopes operated at higher photon energies have been constructed at the Advanced Photon Source [43], at the European Synchrotron Radiation Facility [7], and at SPring-8 [82]. An overview of the operational and planned microscopes may be found in Refs. 81 and 75 .

The new scanning transmission X-ray microscope of the Göttingen Institute for X-Ray Physics operates at the BESSY II electron storage ring [93, 94, 95]. Fig. 2.1 shows a schematic overview of the STXM beamline with the monochromator, the object stage and the detector. The X-ray source for the microscope is the undulator U41 located in a low- $\beta$ straight section of the storage ring (see Sec. 3.1). The microscope is designed for operation in the soft X-ray water window energy region, which is fully covered by the first harmonic radiation of the undulator. The zone plate of the STXM has to be illuminated spatially coherently and with a low spectral bandwidth (see Section 1.2). Hence, the source property which determines the photon rate in the focal spot is the brilliance of the undulator beam, which gives the photon rate per source diameter, beam divergence and spectral bandwidth. The peak brilliance of the first uneven harmonics of 


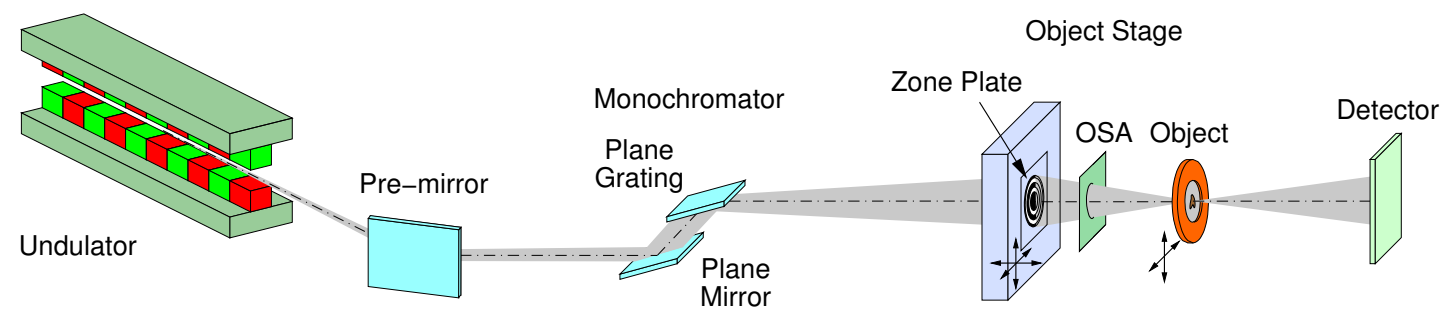

Figure 2.1: Schematic overview of the BESSY II STXM (see text).

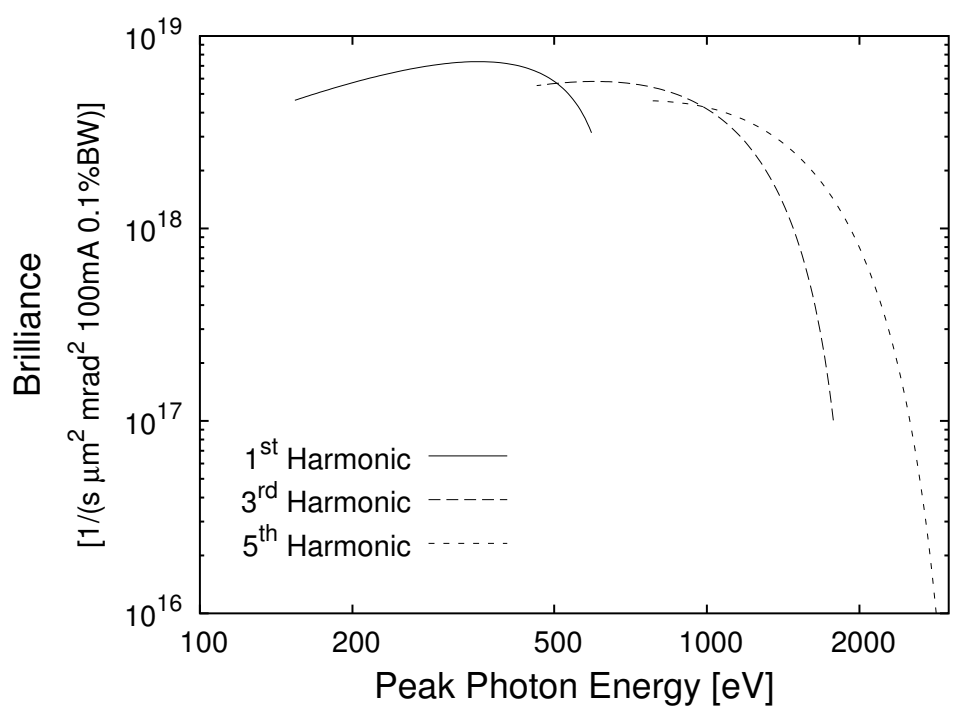

Figure 2.2: Tuning curve: Brilliance of the first, third and fifth harmonic of the undulator U41 with the gap set for peak brilliance at each energy (calculated with SPECTRA [83]).

the undulator U41 is depicted in Fig. 2.2. It exceeds $10^{18}$ Phot./( $\mathrm{sm}^{2} \mathrm{mrad}^{2}$ $100 \mathrm{~mA} 0.1 \% \mathrm{BW}$ ) in the whole water window energy range. This is four orders of magnitude higher than the brilliance of a BESSY I bending magnet where earlier experiments with an STXM were carried out [53,12].

The monochromators of existing soft X-ray STXMs image the undulator or bending magnet source onto a pinhole, which serves as a coherent secondary source for the zone plate illumination [99,89]. Since the area illuminated coherently by the direct beam of the undulator matches the zone plate diameter in the BESSY II STXM, focusing optics and apertures would only decrease the photon rate at the zone plate. Therefore, the monochromator can be built with a very simple setup consisting only of a plane mirror and a plane grating without entrance or exit slits. In addition to the high flux throughput, this design makes the monochromator alignment very easy and the intensity at the zone plate is less susceptible to beam position fluctuations. The line density of the grating is varied to avoid a loss of monochromaticity due to the vertical divergence of the 


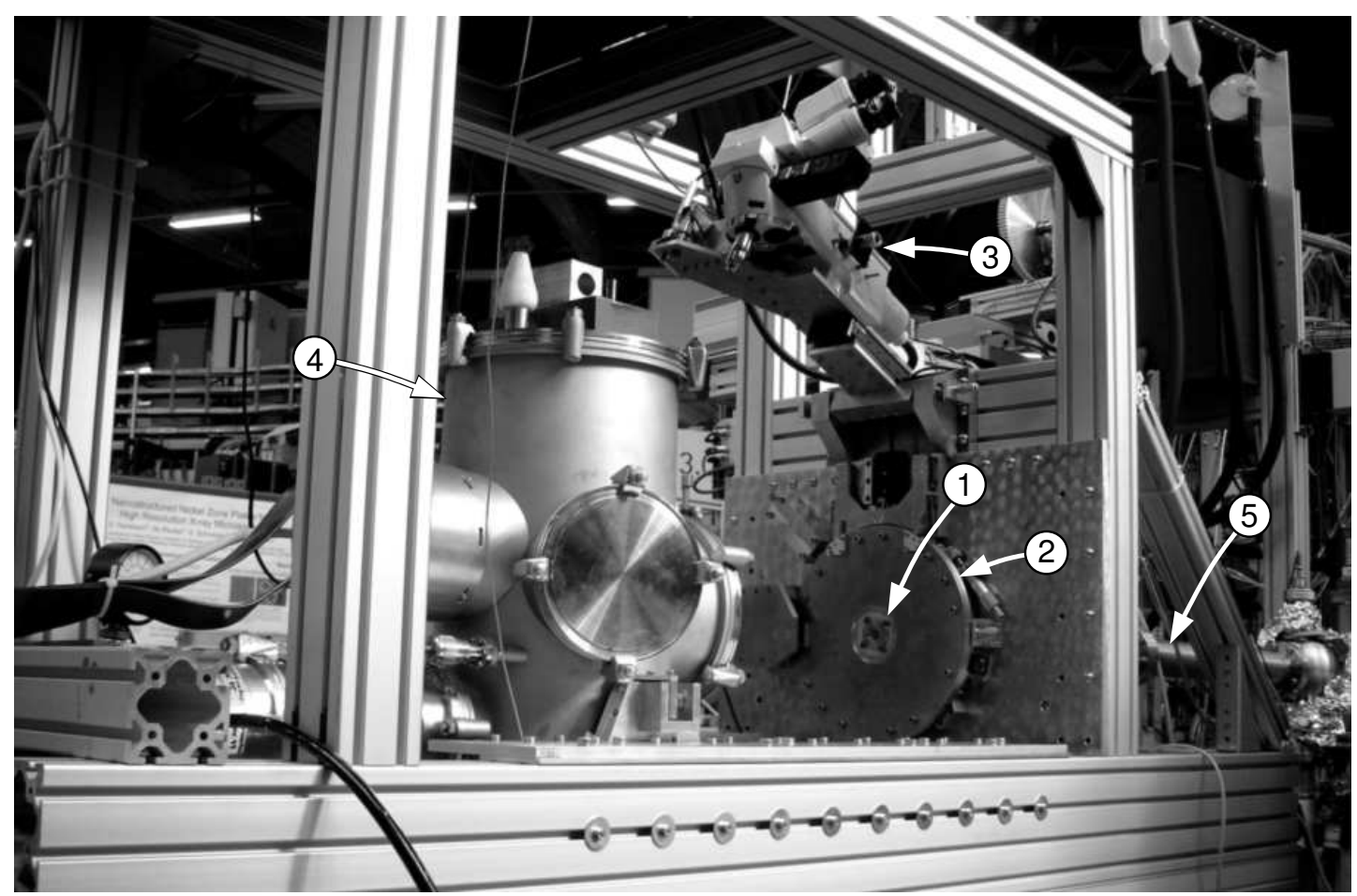

Figure 2.3: Photograph of the scanning transmission X-ray microscope with the specimen chamber (1, see Sec. 4.5), the object stage (2, see Sec. 4.3), the visible light microscope (3), the retracted detector vacuum vessel (4, see Sec. 5.2.1) and the STXM beamline (5, see Sec. 3.3). The zone plate is located in the vacuum vessel behind the object stage.

beam. The operating principle of the monochromator and first test results are described in Chap. 3.

The zone plate selects the desired energy from the vertical fan of light from the grating and focuses it onto the specimen. Currently, nickel zone plates with an outermost zone width of $50 \mathrm{~nm}$ are employed in the STXM (see Sec. 4.2). They have a diameter of $200 \mu \mathrm{m}$ in order to get a large focal length of $2.3 \mathrm{~mm}$ at the carbon absorption edge. A nickel central stop with a diameter of $130 \mu \mathrm{m}$ is galvanized on top of the central zones. Together with the order sorting aperture (OSA) between the zone plate and the specimen, it prevents light of unwanted diffraction orders of the zone plate from reaching the sample.

The specimen is situated in an air gap of a few hundred micrometers between the zone plate and detector vacuum chambers. After retracting the detector vacuum chamber, a visible light microscope can be swiveled in for previewing the sample and for alignment of the OSA with respect to the zone plate (see Fig. 2.3). The specimen is scanned with DC motors to acquire a low resolution image and moved to an interesting position for a high resolution scan. For a high resolution scan, the zone plate is moved with a piezoelectric flexure stage. Because the OSA 
is not moved together with the zone plate, the image field diameter for this high resolution scan is restricted to about $40 \mu \mathrm{m}$ (see Sec. 4.1).

The light transmitted through the specimen is detected by a pn-CCD detector, which is covered in detail in Chap. 5. Typically, $12 \times 12$ pixels of the $64 \times 200$ pixels of the detector are illuminated by the hollow cone created by the zone plate with the central stop. One CCD row is read out in $28.5 \mu \mathrm{s}$, so with a continuous row-byrow readout, the minimum dwell time for the image scan is $0.34 \mathrm{~ms}$ per pixel. By reading out whole CCD frames of the diffraction pattern, one can obtain images with several contrast mechanisms such as dark field or differential phase contrast in addition to the standard incoherent bright field contrast simultaneously with a single scan (see Sec. 1.4.1). 


\section{Chapter 3}

\section{The STXM Monochromator}

The zone plate of a scanning transmission X-ray microscope has to be illuminated both spatially coherently and with a low spectral bandwidth. Therefore, the most important property of the radiation source is not the photon flux but the brilliance. Although there are scanning transmission X-ray microscopes operating at high brilliance bending magnets [2], the ideal source for an STXM is an undulator at a third generation low emittance synchrotron light source. The source for the STXM described in this thesis is the undulator U41 at the BESSY II storage ring. The undulator has been designed specifically for X-ray microscopy in the water window energy region between the oxygen and carbon $\mathrm{K}$ absorption edges, which is fully covered by the first harmonic of the undulator. The monochromaticity of the direct undulator beam is below 100, so a monochromator has to be employed in order to obtain the relative bandwidth needed to get a diffraction limited focal spot of the zone plate (Sec. 1.2) and for NEXAFS spectroscopy (Sec. 1.1.1). The design of the monochromator depends on the properties of the undulator, which is described in the following section.

\subsection{The Undulator U41}

Undulators are composed of $N$ periods of alternating magnet pairs inserted into a straight section of an electron storage ring. The magnetic field forces the electrons of the storage ring onto an undulating trajectory. Because of the relativistic energy of the electrons, the electromagnetic radiation caused by the accelerated motion of the charged electrons is emitted tangentially. The parameter $K$ describes the maximum deflection angle $\delta$ of the electrons depending on their Lorenz factor $\gamma[5]$ :

$$
K=\delta \gamma=\frac{\mathrm{e} \lambda_{0} B_{0}}{2 \pi \mathrm{m}_{\mathrm{e}} \mathrm{c}}
$$

where e and $\mathrm{m}_{\mathrm{e}}$ are the electron charge and mass, $\lambda_{0}$ is the magnet period and $B_{0}$ is the magnetic field. $K$ is varied by adjusting the gap between the opposing magnets which changes the magnetic field. For undulators, $K$ is relatively low 
$(K \lesssim 1)$, so the X-ray amplitudes generated by the different undulations can add up coherently for multiples of a base photon energy, the so called harmonics. Periodic magnet structures with $K \gg 1$ are called wigglers. Because the electrons are forced onto larger undulations, the radiation adds up incoherently leading to a continuous spectrum similar to that of a bending magnet.

The undulator equation describes the $K$ dependence of the wavelength $\lambda$ of the $k^{\text {th }}$ uneven harmonic at the observation angle $\theta$ from the undulator axis [5]:

$$
\lambda=\frac{\lambda_{0}}{2 k \gamma^{2}}\left(1+\frac{K^{2}}{2}+\gamma^{2} \theta^{2}\right)
$$

One obtains a brilliance increase of order $N^{2}$ compared to a single bending magnet. The monochromaticity of the on axis beam of the $k^{\text {th }}$ harmonic of an undulator is about $E / \Delta E \approx k N$ and the half angle of the monochromatic central radiation cone $\theta_{\text {cen }} \approx 1 / \gamma \sqrt{N}[5]$.

For the design of the monochromator and the microscope, the characteristics of the light emitted from the undulator have to be known. They can be calculated from the properties of the storage ring and the undulator with computer codes such as SMUT [32] or SPECTRA [83]. In Tab. 3.1 the properties of the BESSY II storage ring and the undulator U41 used for the calculation of the beam properties are listed. The undulator is installed in a low-beta straight section of the ring which leads to a lower heat load on the first beamline optics and to a rounder source [25]. For the calculations with SPECTRA the Twiss parameters $\alpha_{\mathrm{x}}$ and $\alpha_{\mathrm{y}}$ are neglected because they are close to zero in the middle of a straight section [55].

Fig. 3.1 shows the calculated photon rate of the direct beam of the undulator U41 at the distance of the zone plate for a fixed $K$ value of $K=1.645$. The first undulator harmonic at $E=283 \mathrm{eV}$ and the weaker higher harmonics are visible. The even harmonics are suppressed compared to the odd harmonics. The full width at half maximum of the first harmonic is $\Delta E=4.7 \mathrm{eV}$ corresponding to a monochromaticity of $E / \Delta E=58$.

The spatial distribution of the monochromatic photon flux of the first two harmonics at the distance of the STXM zone plate is shown in Fig. 3.2. where the gap is set for a first harmonic peak energy of $283 \mathrm{eV}$. The intensity of the first harmonic is concentrated on the center maximum. The intensity profile can be approximated by a Gaussian distribution with a full width at half maximum of $d_{\mathrm{h}}=6.3 \mathrm{~mm}$ horizontally and $d_{\mathrm{v}}=2.2 \mathrm{~mm}$ vertically. Most of the intensity of the second harmonic is distributed into the off-axis maxima. This is the reason for the lower flux on the undulator axis for the even harmonics visible in Fig. 3.1.

Fig. 3.3 shows the total power of the undulator radiation from the undulator. At a peak energy of the first harmonic of $283 \mathrm{eV}$, the beam has a power of about $100 \mathrm{~W}$, most of which is absorbed in the first optical elements of the beamline, so they have to be water cooled in order to prevent heat accumulation and distortion of the optics surfaces. 
Table 3.1: Characteristics of the BESSY II storage ring and the undulator U41 [26]

\begin{tabular}{|c|c|c|c|}
\hline \multicolumn{4}{|c|}{ Storage ring } \\
\hline Electron energy & $E$ & $1.7 \mathrm{GeV}$ & \\
\hline Lorenz factor & $\gamma$ & 3327 & \\
\hline Ring circumference & & $240 \mathrm{~m}$ & \\
\hline Typical beam current after injection & $I$ & $260 \mathrm{~mA}$ & \\
\hline Nominal beam current & $I$ & $100 \mathrm{~mA}$ & \\
\hline Typical life time & $t$ & $6 \mathrm{~h}$ & \\
\hline Pulse length & & $18 \mathrm{ps}$ & \\
\hline Revolution time & & $800 \mathrm{~ns}$ & \\
\hline Coupling & & $1.7 \%$ & \\
\hline Natural emittance & $\epsilon$ & $5.4 \times 10^{-9} \pi \mathrm{rad} \mathrm{m}$ & \\
\hline Emittance at $1.7 \%$ coupling & $\epsilon_{\mathrm{h}}$ & $5.3 \times 10^{-9} \mathrm{~m} \mathrm{rad}$ & * \\
\hline & $\epsilon_{\mathrm{V}}$ & $9 \times 10^{-11} \mathrm{~m} \mathrm{rad}$ & * \\
\hline Dispersion & $\eta_{\mathrm{h}}$ & $2 \times 10^{-2} \mathrm{~m}$ & * \\
\hline & $\eta_{\mathrm{h}}$ & $0.01 \mathrm{rad}$ & \\
\hline & $\eta_{\mathrm{v}}$ & $2 \times 10^{-2} \mathrm{~m}$ & \\
\hline & $\eta_{\mathrm{v}}$ & $0.01 \mathrm{~m}$ & * \\
\hline Natural energy spread & $\Delta E / E$ & $0.8 \times 10^{-3}$ & * \\
\hline \multicolumn{4}{|c|}{ Undulator U41 } \\
\hline Number of periods & $N$ & 81 & \\
\hline Period length & $\lambda_{0}$ & $41.2 \mathrm{~mm}$ & \\
\hline Total length & $L$ & $3337.2 \mathrm{~mm}$ & \\
\hline Vacuum gap & $g_{\mathrm{vac}}$ & $11 \mathrm{~mm}$ & \\
\hline Magnet gap & $g_{\mathrm{mag}}$ & $>15 \mathrm{~mm}$ & \\
\hline$K$ parameter range & $K$ & $0.5 \ldots 2.58$ & \\
\hline Photon energy in the $1^{\text {st }}$ harmonic & $\mathrm{h} \nu$ & $596 \mathrm{eV} \ldots 154 \mathrm{eV}$ & \\
\hline Beta function & $\begin{array}{l}\beta_{\mathrm{h}} \\
\beta_{\mathrm{v}}\end{array}$ & $\begin{array}{l}1.117 \mathrm{~m} / \mathrm{rad} \\
1.200 \mathrm{~m} / \mathrm{rad}\end{array}$ & \\
\hline
\end{tabular}

* Measured parameter 


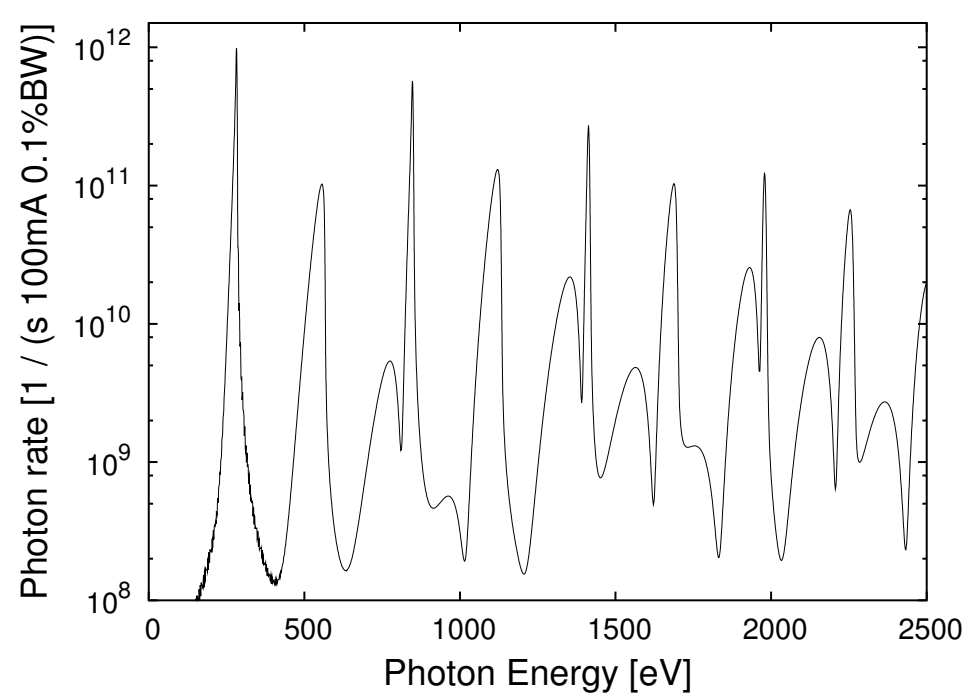

Figure 3.1: Photon rate of the undulator U41 behind an on-axis pinhole with $200 \mu \mathrm{m}$ diameter at a distance of $37 \mathrm{~m}$ from the undulator, calculated with SPECTRA [83]. The peak energy of the first harmonic is $E=283 \mathrm{eV}(K=1.645)$. The full width at half maximum of the first harmonic is $\Delta E=4.7 \mathrm{eV}$.

Table 3.2: Standard deviations of the source diameter and divergence of the radiation emitted by the undulator U41 approximated by gaussian distributions at a photon energy of $283 \mathrm{eV}$ and $524.5 \mathrm{eV}$, calculated with SPECTRA [83].

\begin{tabular}{lcccc}
\hline & & & \multicolumn{2}{c}{ Photon energy } \\
& & & 283 & 524.5 \\
\hline Horizontal source size & $\sigma_{\mathrm{h}}$ & $\mu \mathrm{m}$ & 79 & 79 \\
Vertical source size & $\sigma_{\mathrm{v}}$ & $\mu \mathrm{m}$ & 14 & 13 \\
Horizontal divergence & $\sigma_{\mathrm{h}}^{\prime}$ & $\mu \mathrm{rad}$ & 79 & 75 \\
Vertical divergence & $\sigma_{\mathrm{v}}^{\prime}$ & $\mu \mathrm{rad}$ & 38 & 29 \\
\hline
\end{tabular}

In Fig. 3.4 the size and divergence of the undulator beam approximated by Gaussian distributions is depicted. The source diameter and divergence decreases at higher peak energies of the first harmonic. The values for the oxygen and carbon absorption edges are tabulated in Tab. 3.2. From these parameters the coherently illuminated area can be calculated (Sec. 3.2.2). 

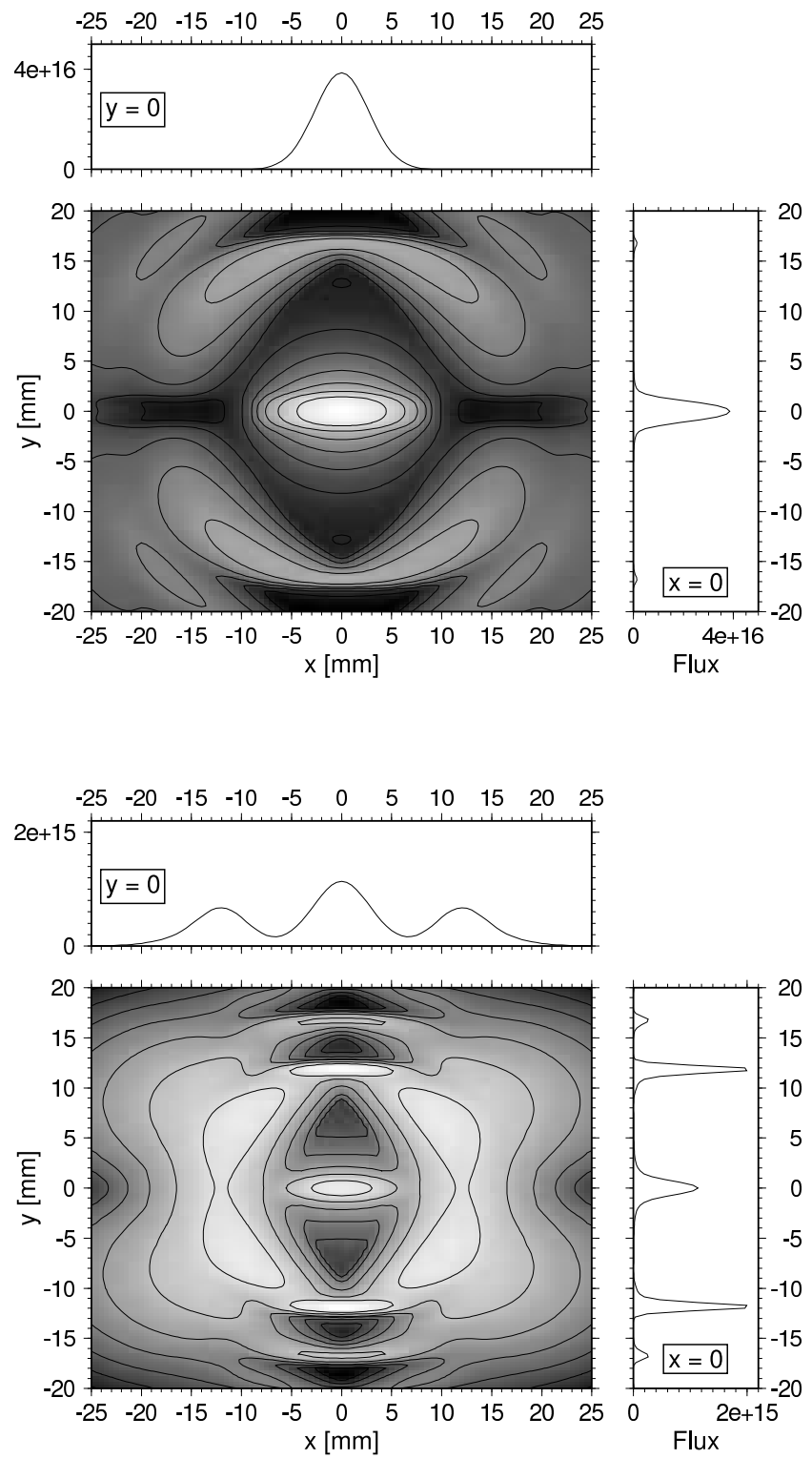

Figure 3.2: Spatial distribution of the monochromatic photon flux in Phot./( $\left.\mathrm{smrad}^{2} 0.1 \% \mathrm{BW}\right)$ at $K=1.645$ at the distance of the zone plate for the undeflected beam, calculated with SPECTRA [83]. Shown are maps of the flux and sections through the map at $x=0$ and $y=0$. Top: flux distribution of the first harmonic at $283 \mathrm{eV}$. Bottom: flux distribution of the second harmonic at $566 \mathrm{eV}$. 


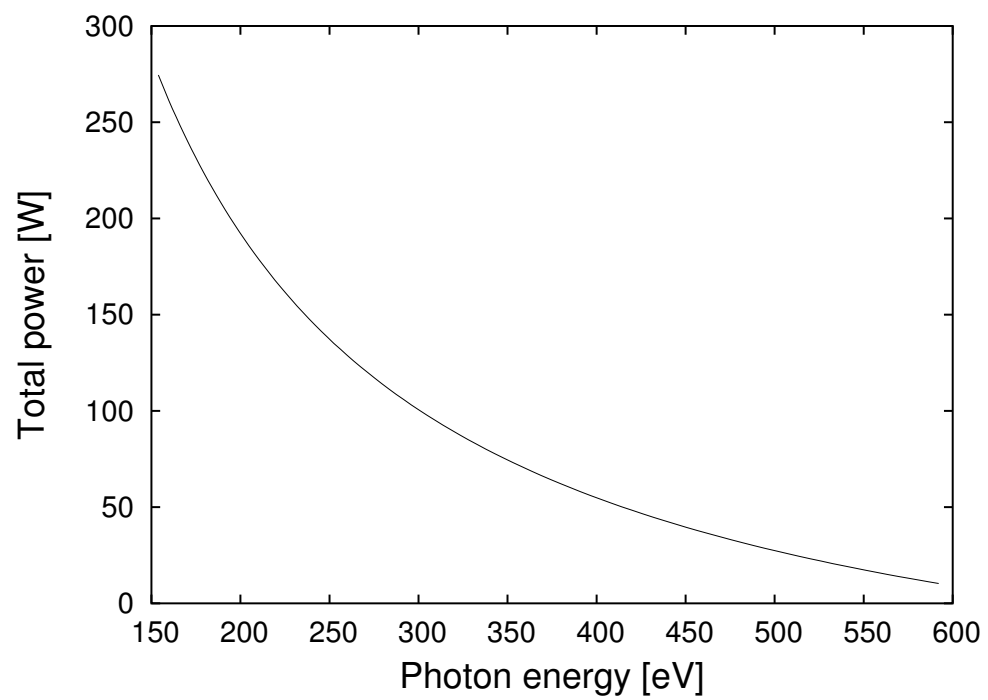

Figure 3.3: Total power emanating from the undulator U41 as a function of the peak energy of the first harmonic, calculated with SPECTRA [83].
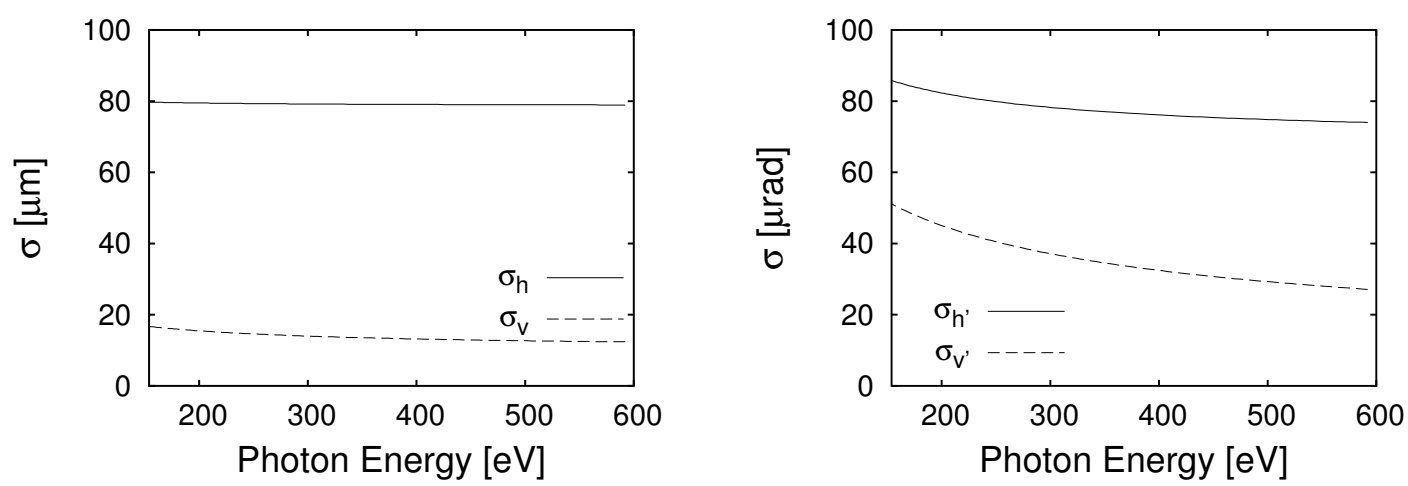

Figure 3.4: Standard deviations of source size (left) and divergence (right) of the undulator U41 approximated by Gaussian distributions as a function of the peak energy of the first harmonic, calculated with SPECTRA [83]. 


\subsection{Principle of Operation of the Monochroma- tor}

The monochromator for the STXM was designed with the following requirements in mind:

- High flux throughput: At a given signal to noise ratio for a spectrum or an image, the time needed for the data acquisition is inversely proportional to the photon rate in the focus. Therefore, the number of optical elements in the beam path has to be minimized and their efficiency has to be as high as possible.

- Moderately high spectral resolution: In order to obtain the diffraction limited diameter of the zone plate focal spot, the relative spectral resolution has to be at least equal to the number of zones, which is 1000 for the currently used zone plates. For NEXAFS spectra a monochromaticity of several thousand is desirable to resolve the absorption lines. A spectral resolution higher than needed decreases the available photon rate.

- Preservation of the spatial coherence: Because the zone plate needs to be illuminated coherently, only a part of the undulator beam may be used.

- Energy reproducibility: The energy selected by the monochromator has to be absolutely calibrated to be able to compare spectra taken from different samples.

- Higher order and higher harmonics suppression: Radiation of higher harmonics of the undulator is diffracted at higher orders of diffraction of the grating and zone plate at the same angle as the first harmonic. For quantitative analysis, their influence has to be determined and minimized.

- Practicability: After switching between the different experiments at the undulator (see Sec. 3.3) or after an injection, the beam position and direction might vary. Therefore, the monochromator has to be easily realigned.

Fig. 3.5 gives a schematic overview of the monochromator principle. It has the simplest possible setup with a plane mirror which sets the incident angle and a plane grating with varied line density as the dispersive element.

The line density of the grating is varied in order to compensate the divergence of the beam (see Sec. 3.2.1). The beam is not focused in the horizontal direction, because the spatially coherently illuminated area closely matches the zone plate diameter. The varied line density of the grating makes it possible to accept the full undulator beam with the monochromator, so there is no need for an entrance slit. There is also no exit slit; the zone plate selects the radiation of the desired energy. The absence of entrance or exit slits makes the illumination of the zone plate less 


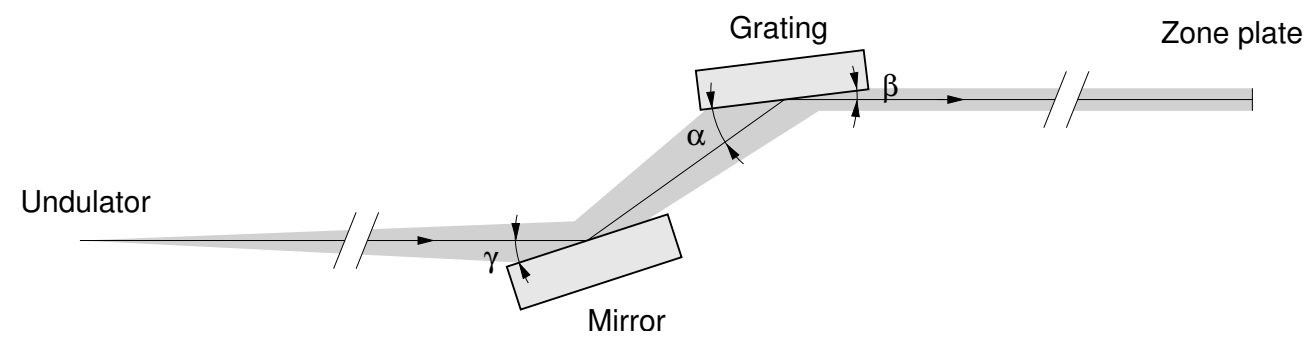

Figure 3.5: Schematic monochromator principle with the plane mirror and the plane grating. The BESSY switching mirror is not shown.

sensitive to fluctuations of the beam position and allows a simple alignment of the monochromator.

For smaller changes of the energy, for example to take a NEXAFS spectrum over an absorption edge, only the grating is turned at a constant angle of deflection. When changing the energy by a larger step, for example for working at another absorption edge, the angle of deflection is adjusted by turning the mirror.

This design is based on an earlier setup which was developed for the STXM at BESSY I $[11,12]$. The crucial element of the monochromator is the diffraction grating, which determines the achievable monochromaticity.

\subsubsection{The Diffraction Grating}

The gratings for the monochromator were built at the Institute for X-Ray Physics by R. Früke [21]. They are designed for operation in the water window energy range and optimized for the carbon absorption edge, where the majority of the experiments will be carried out. The laminar gratings are exposed holographically. Gratings with mean line densities of 600 lines/mm and 1200 lines $/ \mathrm{mm}$ have been built. Fig. 3.6 shows the diffraction efficiency of a 600 lines $/ \mathrm{mm}$ grating measured by R. Früke and G. Reichardt (BESSY) and of a 1200 lines/mm grating measured by F. Scholze (PTB) at different reflectometer beamlines at BESSY II [21]. The efficiency of the 1200 lines/mm grating decreases much steeper at higher energies than the efficiency of the 600 lines/mm grating.

The vertical source size of the undulator is by a factor of three lower than the horizontal size (see Fig. 3.4). Since the achievable spectral resolution of the monochromator is limited by the source size, the vertical plane is selected as the dispersion plane.

The divergent light from the undulator impinges on the grating with different angles of incidence $\alpha$ at different positions, whereas the diffraction angle $\beta$ for the $\mathrm{X}$-rays reaching the zone plate is approximately constant over the whole grating (see Fig. 3.5). For a given diffraction order $m$ and grating constant $d$, the grating equation

$$
\cos \alpha-\cos \beta=\frac{m \lambda}{d}
$$




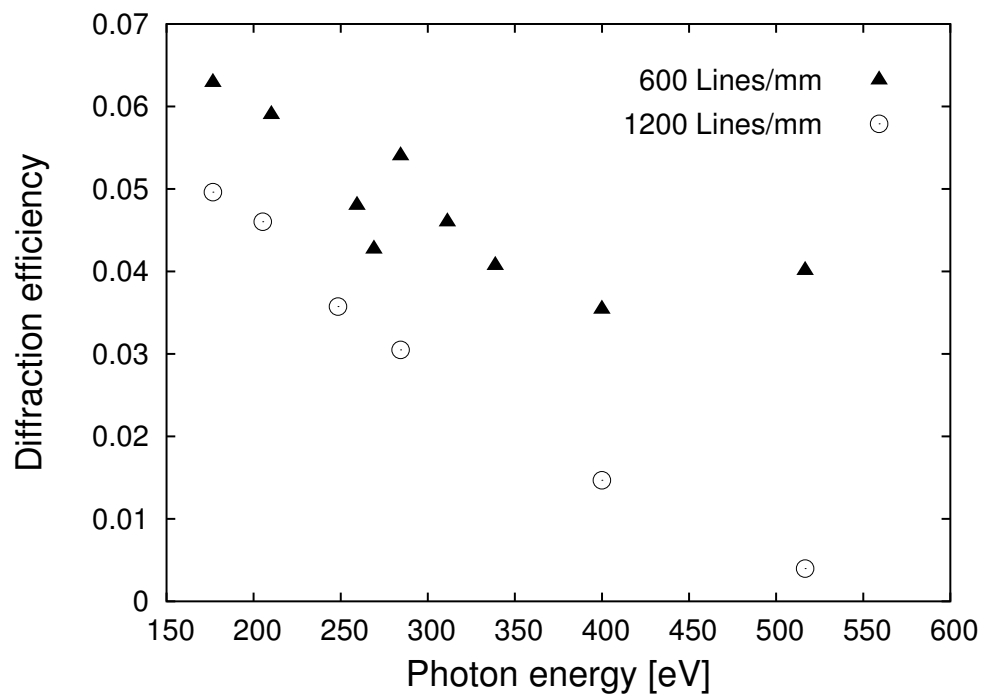

Figure 3.6: Measured diffraction efficiency of monochromator gratings with a mean line density $d^{-1}$ of 600 lines $/ \mathrm{mm}$ and 1200 lines/mm [21].

holds for a whole range of different wavelengths $\lambda$ at different angles of incidence. In order to keep the wavelength of the diffracted radiation constant, the grating constant $d$ has to be varied along the grating. For a certain incident angle $\alpha$ which depends on the wavelength, the diffraction angle is then constant over the whole grating and the monochromaticity is not diminished by X-rays of different wavelengths reaching the zone plate. Tab. 3.3 lists the properties of the gratings employed in the monochromator.

There are more factors influencing the monochromaticity such as the finite source size of the undulator or the slope errors of the mirrors and the grating which have to be taken into account when estimating the monochromaticity with ray tracing calculations. Fig. 3.7 shows the calculated monochromaticity for the two line densities. For all energies, the grating with higher line density yields a higher monochromaticity. With the 1200 lines/mm grating, a monochromaticity of about 3500 is expected at the carbon absorption edge compared to 2500 for the grating with 600 lines $/ \mathrm{mm}$. Therefore, the 1200 lines $/ \mathrm{mm}$ grating is installed in the monochromator despite the lower but still sufficient diffraction efficiency of $3.0 \%$ at the carbon absorption edge $(284 \mathrm{eV})$ and $0.5 \%$ at $517 \mathrm{eV}$ (see Fig. 3.6).

By differentiating the grating equation (3.2) with respect to the diffraction angle $\beta$ one obtains for the dispersion of the grating:

$$
\frac{\mathrm{d} \beta}{\mathrm{d} \lambda}=\frac{m}{d \sin \beta}
$$

If one changes the wavelength by one monochromaticity step $\Delta \lambda=\lambda / 3500$ at the carbon absorption edge for a 1200 Lines/mm grating, the height of the beam at the distance of the zone plate of $d_{\mathrm{ZP}}=16.5 \mathrm{~m}$ changes by $\Delta h=d_{\mathrm{ZP}} \Delta \beta=1.4 \mathrm{~mm}$. 
Table 3.3: Properties of the laminar gratings employed in the STXM monochromator with the incident and diffraction angles from the grating surface at $283 \mathrm{eV}$ and at $524.5 \mathrm{eV}$ (from Ref. 21).

\begin{tabular}{|c|c|c|c|c|}
\hline & & & \multicolumn{2}{|c|}{$\begin{array}{c}\text { Mean line density } d^{-1} \\
\text { Lines } / \mathrm{mm}\end{array}$} \\
\hline & & & 600 & 1200 \\
\hline Lower line density & $d_{0}^{-1}$ & Lines/mm & 598.65 & 1197.32 \\
\hline Upper line density & $d_{1}^{-1}$ & Lines/mm & 601.35 & 1202.69 \\
\hline Length of grating & $l$ & $\mathrm{~mm}$ & & 44 \\
\hline Diffraction order & $m$ & & & -1 \\
\hline \multirow[t]{2}{*}{ Incident angle } & $\alpha(283 \mathrm{eV})$ & & $4.228^{\circ}$ & $5.963^{\circ}$ \\
\hline & $\alpha(524.5 \mathrm{eV})$ & & $3.105^{\circ}$ & $4.376^{\circ}$ \\
\hline \multirow[t]{2}{*}{ Diffraction angle } & $\beta(283 \mathrm{eV})$ & & $0.782^{\circ}$ & $1.002^{\circ}$ \\
\hline & $\beta(524.5 \mathrm{eV})$ & & $0.569^{\circ}$ & $0.720^{\circ}$ \\
\hline Line-to-space ratio & $\xi$ & & 0.76 & 0.86 \\
\hline Groove depth & $h$ & $\mathrm{~nm}$ & 16 & 11 \\
\hline Nickel coating thickness & $t$ & $\mathrm{~nm}$ & & 30 \\
\hline
\end{tabular}

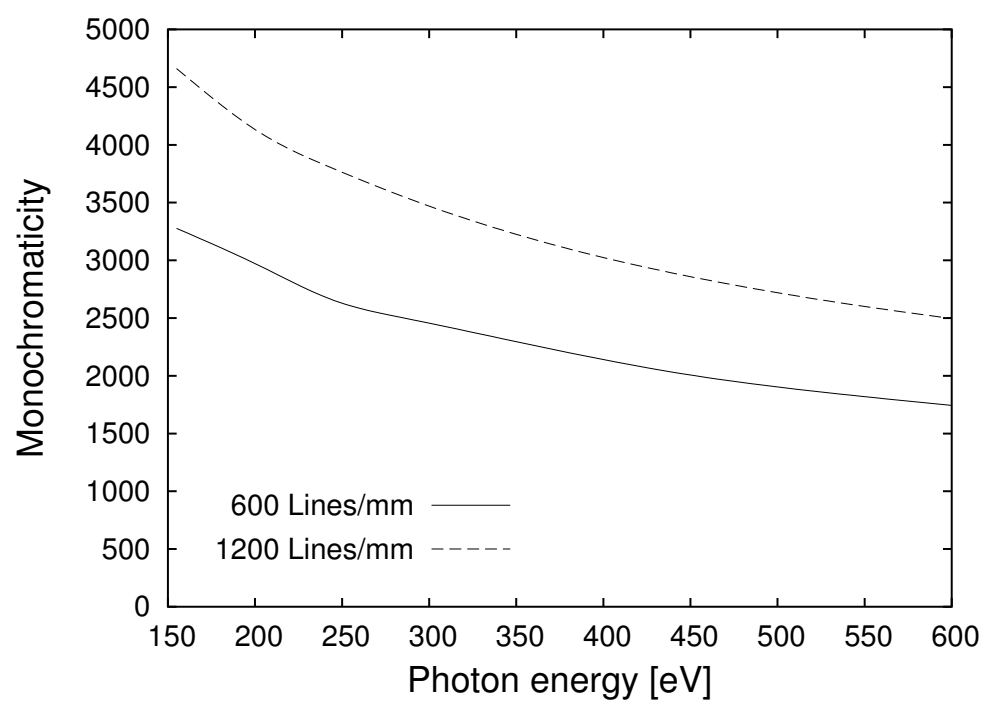

Figure 3.7: Calculated monochromaticity of the STXM monochromator with a grating with 600 lines/mm and 1200 Lines/mm [21]. 
Hence, the whole zone plate height is illuminated monochromatically and the zone plate may be moved vertically for image scans of heights below $1 \mathrm{~mm}$ without a visible change of the wavelength.

The horizontal divergence of the undulator beam is not affected by the monochromator, so the beam width at the zone plate is about $6.3 \mathrm{~mm}$ wide and the zone plate may also be moved horizontally for the image scans.

\subsubsection{Spatial Coherence of the Zone Plate Illumination}

In order to get a diffraction limited focal spot size, the illumination of the zone plate has to be spatially coherent, or, in other words, the source has to be indiscernible from a point source. For a Gaussian profile of the source with half $1 /$ e diameter $\sigma_{\mathrm{h}}$ and half $1 / \mathrm{e}$ divergence $\sigma_{\mathrm{h}}^{\prime}$, the limit for an acceptable degree of coherence of 0.88 is [55]:

$$
\sigma_{\mathrm{h}} \sigma_{\mathrm{h}}^{\prime}=\frac{\lambda}{2 \pi}
$$

At a photon energy of $283 \mathrm{eV}$ the vertical source size is $\sigma_{\mathrm{h}}=14 \mu \mathrm{m}$ (see Tab. 3.2), so maximum source acceptance is $\sigma_{\mathrm{h}}^{\prime}=50 \mu \mathrm{rad}$ and the full beam with a divergence of $\sigma_{\mathrm{h}}^{\prime}=38 \mu \mathrm{rad}$ may be used. Even at $524.5 \mathrm{eV}$, the full beam with maximum divergence of 29 prad is still spatially coherent.

Because of the horizontal source size of $\sigma_{\mathrm{h}}=79 \mu \mathrm{m}$, the maximum angular acceptance at $283 \mathrm{eV}(524.5 \mathrm{eV})$ is $\sigma_{\mathrm{h}}^{\prime}=8.8 \mu \mathrm{rad}(4.8 \mu \mathrm{rad})$. From the undulator, the zone plate appears under a half angle of $2.6 \mu \mathrm{rad}$, so the full horizontal width of the beam may be used.

\subsubsection{Spectral Contamination by Higher Undulator Har- monics}

According to the grating equation (3.2), radiation of higher harmonics $k$ of the undulator with wavelengths inversely proportional to $k$ is diffracted at higher diffraction orders $m=k$ at the same angle. This also holds for the zone plate, where higher harmonics radiation is focused with the same focal length in diffraction order $k$. Therefore, unwanted radiation of higher energies reaches the sample and falsifies the spectra obtained from it. The by a factor of $k$ higher charge generation of the higher energy photons in the detector adds to this effect.

In order to minimize the influence of the higher order spectral contamination, the monochromator mirror is divided lengthwise in two sections coated with nickel and chromium, respectively. Depending on the energy the microscope is working at, the part of the mirror with the appropriate coating is moved into the beam. The energy dependence of the reflectivity of the two mirror coatings is depicted in Fig. 3.8. For working at the carbon absorption edge, the chromium coating is used which cuts off the radiation above the chromium $\mathrm{L}_{3}$ absorption edge at about $570 \mathrm{eV}$. For experiments at the oxygen absorption edge, the nickel coated 


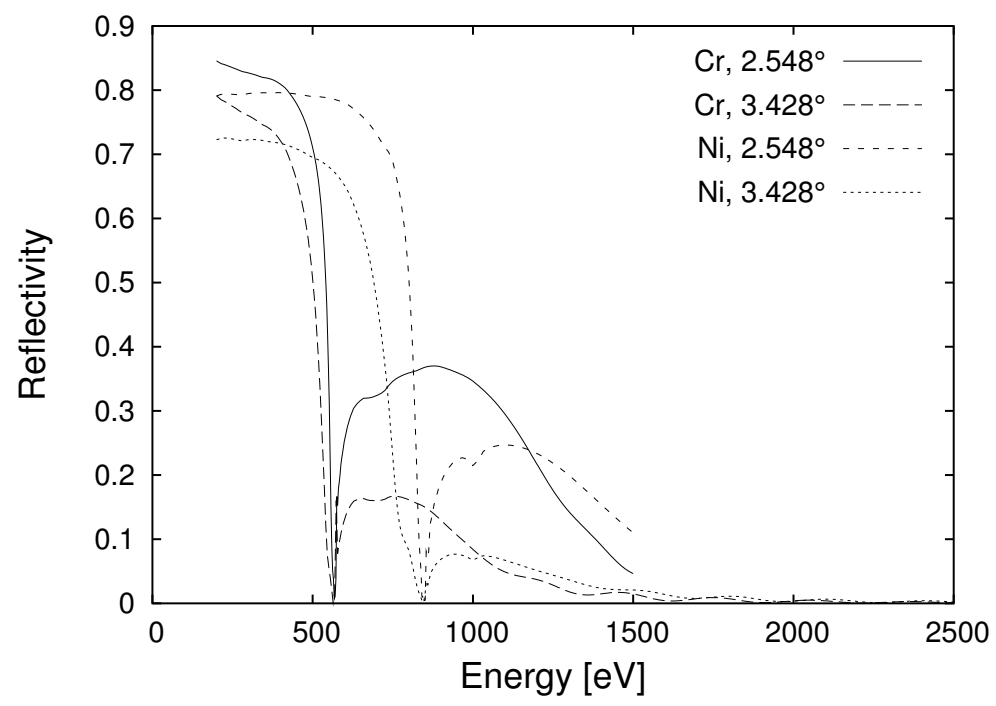

Figure 3.8: Reflectivity of the nickel and chromium mirror coatings at an incidence angle of $2.548^{\circ}$, which is set for a photon energy of $524.5 \mathrm{eV}$, and $3.428^{\circ}(283 \mathrm{eV})$. Data from Ref. 31.

part of the mirror is moved into the beam giving rise to a cutoff above the nickel $\mathrm{L}_{3}$ absorption edge at about $850 \mathrm{eV}$.

\subsection{The STXM Beamline}

The X-ray beam of the undulator U41 is shared by the X-ray microscopy beamline U41-XM and the BESSY microspectroscopy beamline U41-PGM [38] (see Fig. 3.9). In the first switching mirror unit the beam is deflected into one of the two beamlines. The next optical element in the microscopy beamline is the STXM monochromator, which can be shifted out to allow the direct beam to pass through to the TXM or the experimental test chamber. The second switching mirror unit switches the beam among the TXM, the STXM and the experimental test chamber. The experimental test chamber will be used for the calibration of optics and of detectors for the microscopes. It can use either the direct beam with the full bandwidth of the undulator or the low-bandwidth beam with the monochromator moved in. The beam for the TXM is monochromatized using the rotating condenser-monochromator (see Sec. 1.3) The STXM lies in the straight path from the first switching mirror. About $2 \mathrm{~m}$ upstream of the STXM, a beam monitor is installed in the beamline (see Sec. 3.3.1). In the beamline just before the STXM space is provided where a vacuum chamber for cross-linking polymers with the X-ray beam for the zone plate fabrication [91] may be installed.

Fig. 3.10 shows a schematic beamline of the STXM beamline. The beam coming from the undulator U41 can be collimated horizontally and vertically by 


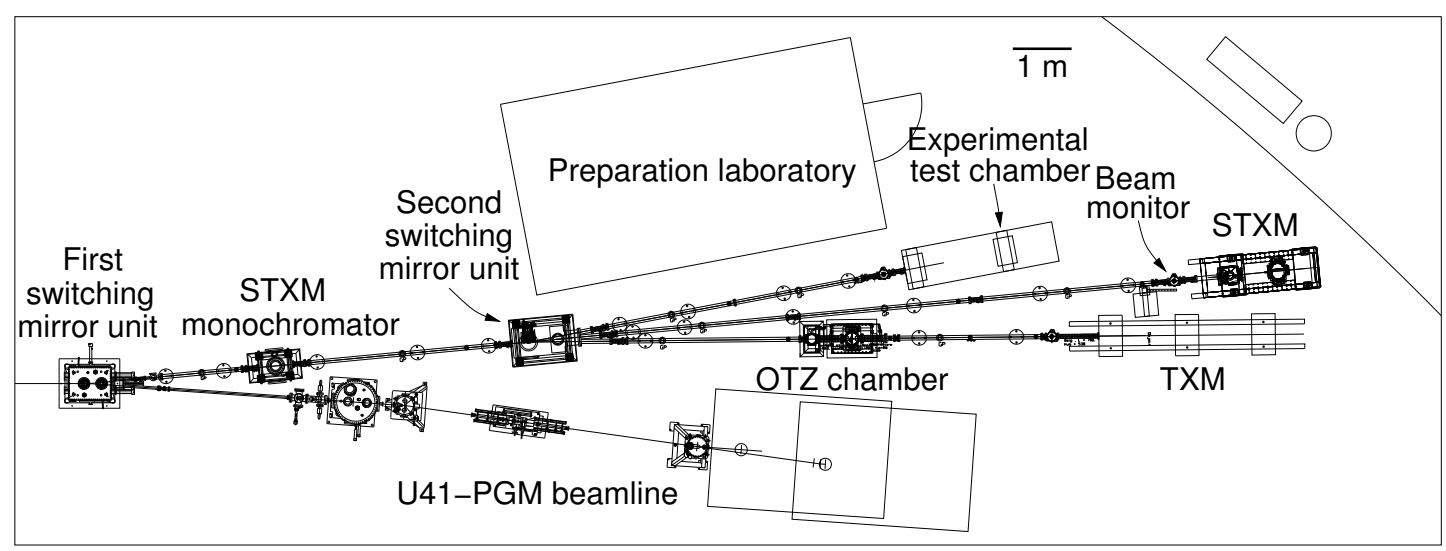

Figure 3.9: Floor plan of the beamlines at the undulator U41: The U41-XM X-ray microscopy beamline (above) with the experimental test chamber, the scanning transmission X-ray microscope (STXM) and the transmission X-ray microscope (TXM), and the U41-PGM beamline (below) [25].
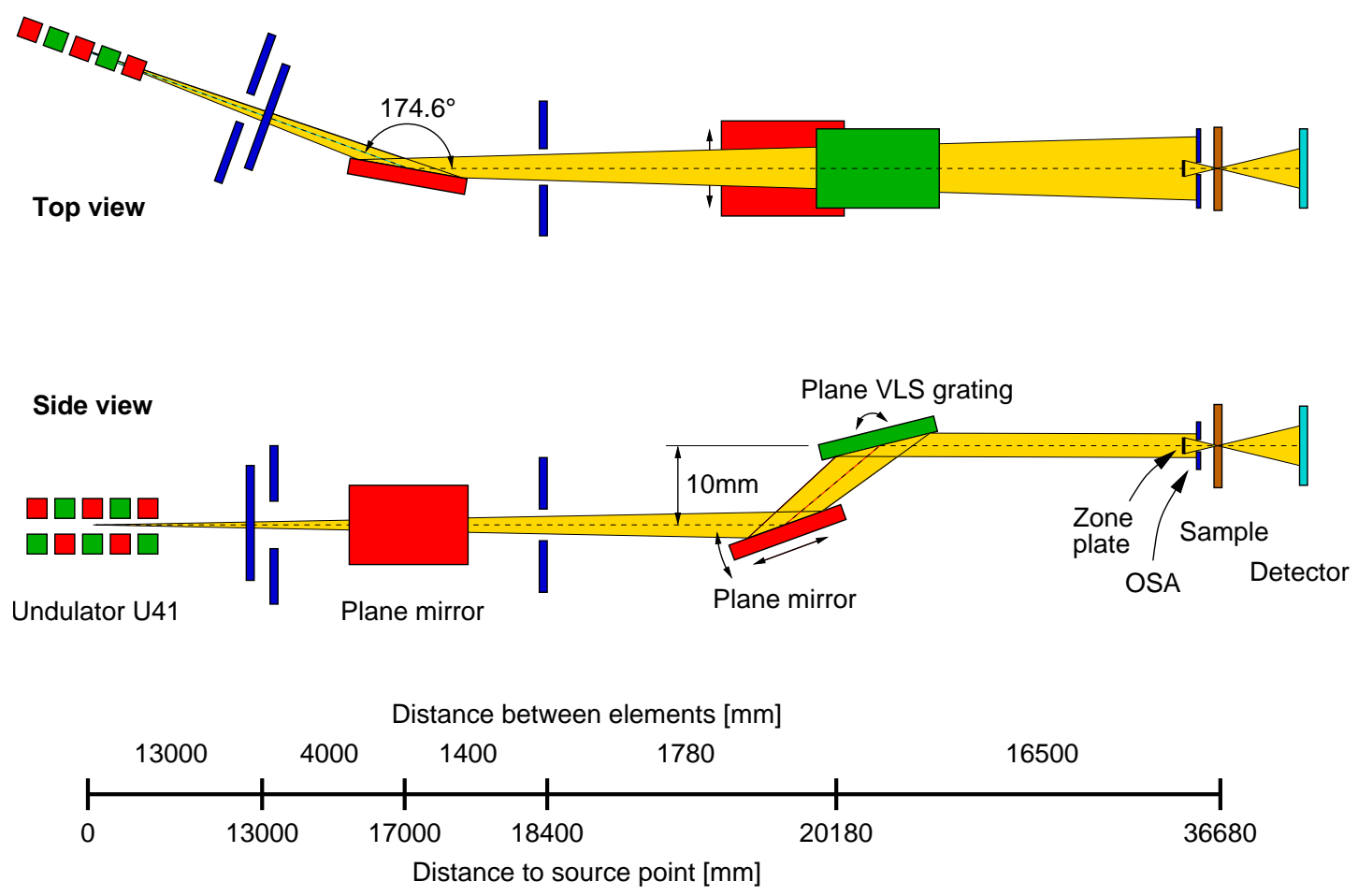

Figure 3.10: Schematic diagram of the STXM beamline. 
four sheet metals in order to reduce the photon rate. This can be necessary to reduce the degradation of the vacuum by the X-rays at high beam currents or low gaps, where the total power is very high (see Fig. 3.3). Angle and position of the nickel coated plane mirror in the first switching mirror unit are adjustable from the outside of the vacuum chamber. About $1 \mathrm{~m}$ behind the first switching mirror unit, a pinhole with a diameter of $1 \mathrm{~mm}$ or $10 \mathrm{~mm}$ may be inserted into the beam. It is used to mask out the off-axis part of the undulator beam which would otherwise pass through the monochromator between the mirror and grating. The monochromator vacuum chamber is located at a distance of $20.18 \mathrm{~m}$ from the undulator. The beam is elevated by $10 \mathrm{~mm}$ by the monochromator. The mechanical setup of the monochromator is described in detail in Sec. 3.4. The vertical fan of light from the grating reaches the STXM $16.5 \mathrm{~m}$ away from the monochromator. The radiation is focussed by the zone plate onto the sample; the detector measures the transmitted light intensity. The zone plate and object stage and the detector are covered in detail in Chapters 4 and 5.

\subsubsection{The Beam Monitor}

The beam position at the microscope may be checked with a beam monitor (Fig. 3.9). It consists of two sheet metals with horizontal and vertical slit apertures of $0.2 \mathrm{~mm}, 1 \mathrm{~mm}$ and $3 \mathrm{~mm}$ width which can be inserted into the beam path with linear vacuum feedthroughs with micrometer screws. They are coated with an UHV-compatible fluorescent coating $\left(\mathrm{Gd}_{2} \mathrm{O}_{2} \mathrm{~S}: \mathrm{Tb}\right.$, Honeywell Lumilux green B43-5). The position of both the direct beam with the monochromator shifted out of the beam and of the vertical line created by the fan of light from the monochromator grating can be viewed through a vacuum window. The position scales of the apertures are calibrated with respect to the beam reference position, so that the beam position can be adjusted by changing the position and angles of the first switching mirror. The beam monitor is also important for checking quickly whether radiation reaches the microscope even when the zone plate and OSA are not adjusted yet and the detector is not running.

\subsection{Mechanical Setup of the Monochromator}

The STXM monochromator has been designed and built by the mechanical workshop of the Institute for X-Ray Physics. The monochromator is located in a ultra high vacuum vessel with $500 \mathrm{~mm}$ diameter. Because great care has been taken to keep the floor of the BESSY experimental area free from vibrations, the vacuum vessel is mounted with four adjustable feet on a concrete block without further vibration isolation. The whole monochromator apparatus with all motion and liquid feedthroughs is mounted on the top DN 250 CF flange of the vacuum chamber (see Fig. 3.11). Therefore, the optics can be mounted and adjusted be- 


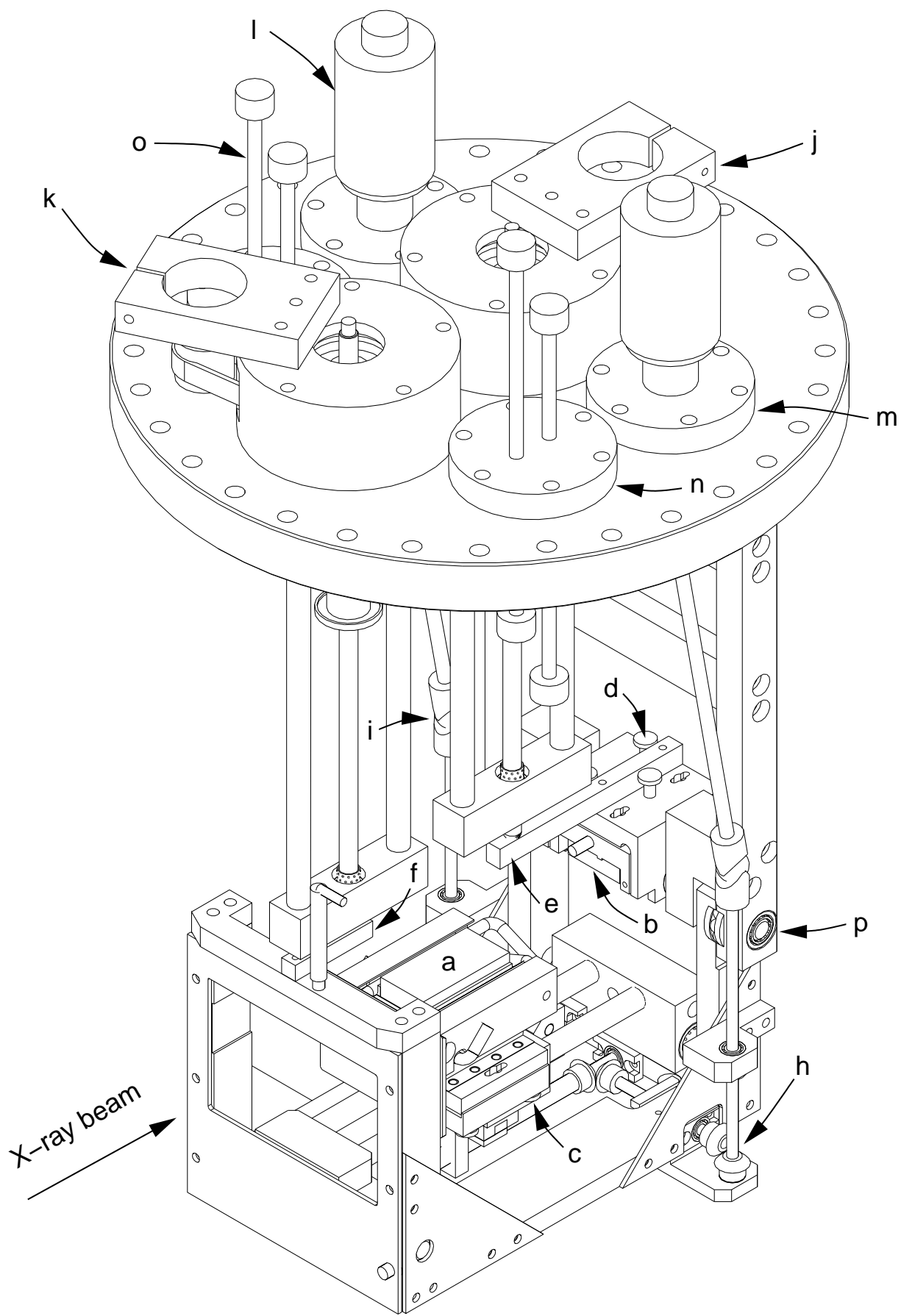

Figure 3.11: Drawing of the STXM monochromator mounted on the top flange [92]. a: mirror, b: grating, c: screws for mirror adjustment, d: screws for grating adjustment, e: grating sine bar, f: mirror sine bar, h: drive for mirror longitudinal translation, $\mathrm{i}$ : drive for mirror transversal translation, $\mathrm{j}$, k: motor drive for grating and mirror rotation (motors not shown), l, m: rotary motion feedthroughs for mirror translation, $\mathrm{n}$, o: liquid feedthroughs for water cooling of mirror and grating, p: mirror axle. 
fore bringing the monochromator into the vacuum chamber. At the front side of the vacuum chamber, an electric feedthrough is mounted for the electrical connections to the Pt100 temperature sensors of the mirror and the grating and to the limit switches.

The grating is pressed by springs onto a water cooled copper holder. The position of the holder can be adjusted with respect to a stainless steel block, which is turned around the grating axis by a custom linear motion feedthrough pushing on a sine bar. The high precision spindle of the feedthrough is moved by a nut driven by a Faulhaber 3564 K 024 BC motor with integrated motion controller via a tooth belt. A digital Heidenhain encoder measures the vertical position of the spindle.

The mirror is rotated eccentrically around an axis below the grating axis (see Sec. 3.4.1). The whole U-shaped support structure for the mirror is turned by a linear motion feedthrough identical to the grating feedthrough pushing on a sine bar. Two water cooled copper blocks are pressed against the side faces of the mirror. The mirror pitch, roll and height on the mirror holder can be adjusted with three screws. It can be translated in the longitudinal and in the transverse direction parallel to the mirror surface plane by spindles which are driven by manual rotary motion feedthroughs.

To avoid carbon contamination on the optics surfaces, the vacuum conditions should be better than $10^{-9}$ mbar without beam. All in-vacuum parts of the monochromator are made of ultra high vacuum compatible material and baked out at $200^{\circ} \mathrm{C}$ before mounting to keep the outgassing rate as low as possible. To avoid virtual leaks all bores are vented. After each venting of the vacuum chamber, the monochromator is baked out at a temperature of $120^{\circ} \mathrm{C}$ for a few days. Without beam, a pressure of about $5 \times 10^{-10}$ mbar is reached, which rises to a few $10^{-8}$ mbar with the beam switched on depending on the undulator $K$ value.

\subsubsection{The Principle of the Mirror Motion}

When the mirror is turned, it also has to be translated along the incoming beam axis so that the beam still hits the grating center. This setup is difficult to realize because there would be a high precision rotating stage on top of a translational stage. If the mirror is turned around an axis below the grating axis, the mirror has to be shifted in the plane of the mirror surface. A sufficiently long mirror does not have to be translated at all. The functional principle of this setup is illustrated in Fig. 3.12.

Ideally, the beam would hit the center of the grating for all angles of incidence, which means that $x_{\mathrm{s}}=0$ for all $\gamma$. If the mirror is turned around the axis at $(X, Y)$, the point of intersection of the beam from the mirror with the straight 


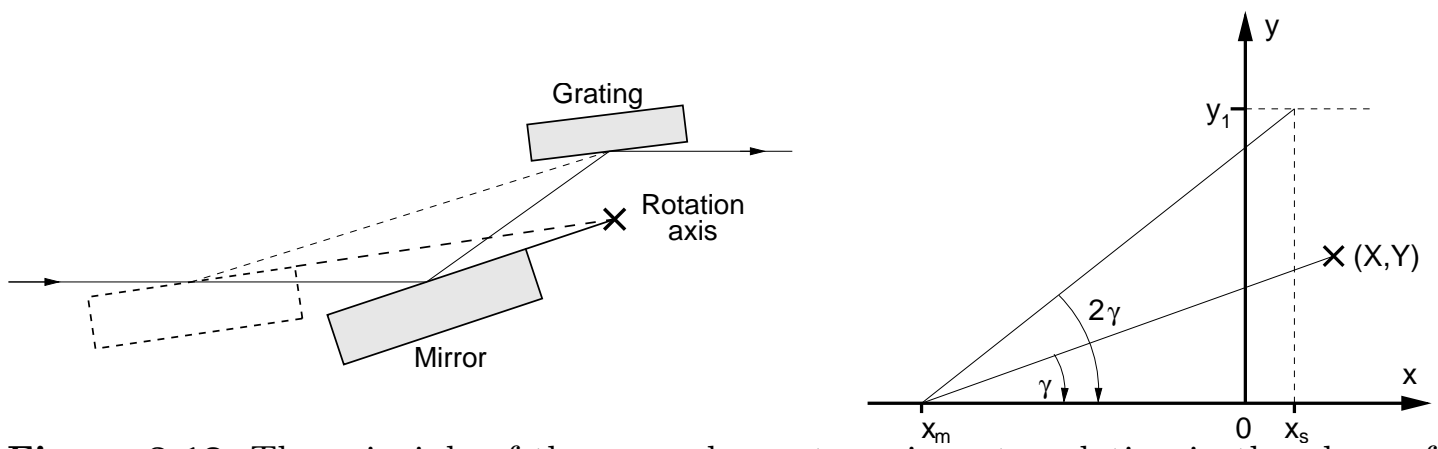

Figure 3.12: The principle of the monochromator mirror translation in the plane of the mirror surface. The beam comes from the left and hits the mirror at $\left(x_{\mathrm{m}}, 0\right)$. The mirror rotates around the axis at $(X, Y)$, the grating around the axis at $\left(0, y_{1}\right)$. The mirror angle is $\gamma$, the angle of the deflected beam is $2 \gamma$. The deflected beam reaches the height of the grating center $\left(y=y_{1}\right)$ at $x=x_{\mathrm{s}}$.

line $y=y_{1}$ is:

$$
x_{\mathrm{s}}(\gamma)=x_{\mathrm{m}}+\frac{y_{1}}{\tan 2 \gamma}=X-\frac{Y}{\tan \gamma}+\frac{y_{1}}{\tan 2 \gamma}
$$

$\gamma_{1}$ and $\gamma_{2}$ are the lower and upper limit for the mirror angle $\gamma$. At the carbon edge $\left(\gamma_{0}=3.474^{\circ}\right)$, the beam should hit the grating center exactly:

$$
x_{\mathrm{s}}\left(\gamma_{0}\right)=0 \Rightarrow X=\frac{Y}{\tan \gamma_{0}}-\frac{y_{1}}{\tan 2 \gamma_{0}}
$$

The deviation from the target position should be equal at both limit angles:

$$
x_{\mathrm{s}}\left(\gamma_{1}\right)=x_{\mathrm{s}}\left(\gamma_{2}\right) \Rightarrow Y=y_{1} \frac{\frac{1}{\tan 2 \gamma_{1}}-\frac{1}{\tan 2 \gamma_{2}}}{\frac{1}{\tan \gamma_{1}}-\frac{1}{\tan \gamma_{2}}}
$$

With the parameters for the STXM monochromator, the optimum mirror axis position is at the half height of the grating axis and displaced vertically by about $0.4 \mathrm{~mm}$ in the direction of the undulator (see Tab. 3.4).

Over the whole $\gamma$ range, the beam should impinge on the grating as closely as possible to the grating center. The distance $\mu$ of the intersection of the beam with the grating from the grating center is given by

$$
\mu=\frac{x_{\mathrm{m}}-\frac{y 1}{\tan 2 \gamma}}{\cos \beta-\frac{\sin \beta}{\tan 2 \gamma}}=\frac{X-\frac{Y}{\tan \gamma}-\frac{y 1}{\tan 2 \gamma}}{\cos \beta-\frac{\sin \beta}{\tan 2 \gamma}}
$$

The $\gamma$ dependence of the distance from the center is shown in Fig. 3.13. Over the whole range of the mirror angle, the deviation is below $20 \mu \mathrm{m}$, which is negligible compared to the grating length of $44 \mathrm{~mm}$. 
Table 3.4: The position of the mirror axis of the monochromator with the parameters used for the calculation.

\begin{tabular}{lccc}
\hline Beam shift & $y_{1}$ & {$[\mathrm{~mm}]$} & 10 \\
Minimum mirror angle & $\gamma_{1}$ & {$\left[^{\circ}\right]$} & 1.9 \\
Maximum mirror angle & $\gamma_{2}$ & {$\left[^{\circ}\right]$} & 3.8 \\
Most frequently used mirror angle & $\gamma_{0}$ & {$\left[^{\circ}\right]$} & 3.474 \\
Optimal mirror axis position & $X$ & {$[\mathrm{~mm}]$} & -0.395 \\
& $Y$ & {$[\mathrm{~mm}]$} & 5.008 \\
\hline
\end{tabular}

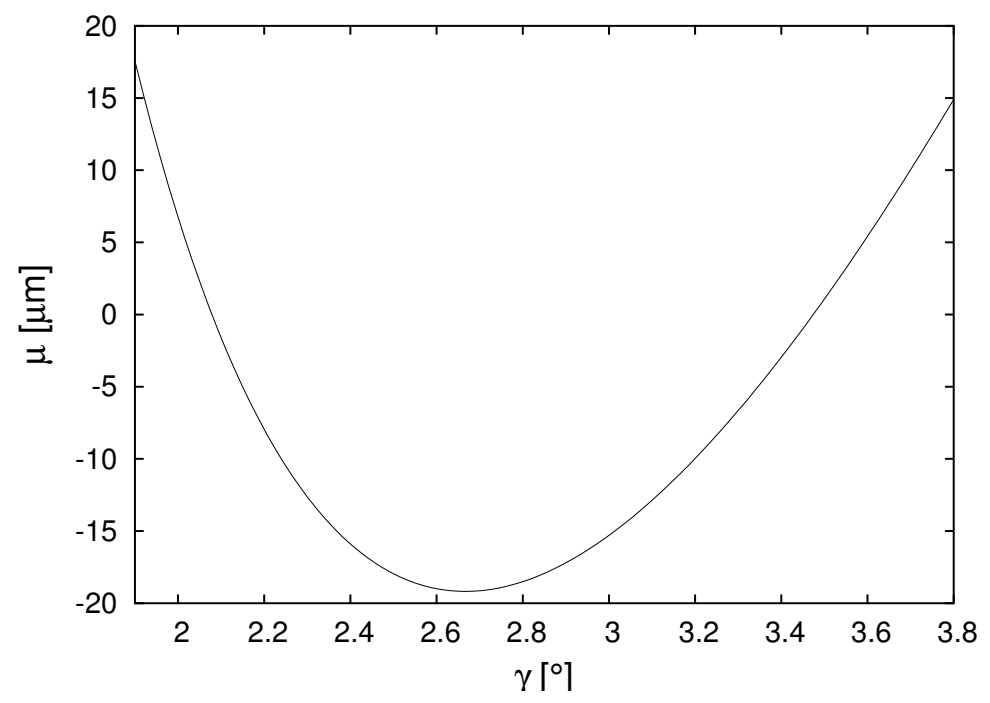

Figure 3.13: Deviation $\mu$ of the beam impinging on the grating from the center. The grating angle is $\beta=0.999^{\circ}$, which corresponds to a photon energy of $283 \mathrm{eV}$. 


\subsubsection{The Alignment of the Monochromator}

The monochromator is aligned according to the following procedure:

1. The monochromator vacuum vessel is centered horizontally and vertically with a theodolite with respect to the optical axis marked on the floor and to the beam height marked at the storage ring wall.

2. The height of the vacuum vessel is adjusted with the help of a levelling telescope.

3. After the assembly of the vacuum parts of the monochromator on the top flange in the clean room, the mirror and the grating are adjusted to their reference positions.

4. Before mounting the monochromator in the vacuum vessel, an adjustable mirror on the monochromator top flange pointing downstream is aligned parallel to the monochromator frame for the later alignment of the vacuum vessel with a diode laser in step 8.

5. When the monochromator is mounted in the vacuum chamber, the position and inclination of the grating and the mirror are controlled with a levelling telescope whether they have changed during the installation.

6. The vacuum vessel is adjusted for horizontal tilt angle with a high precision spirit level.

7. For a fixed angle of the mirror and the grating, the angle is measured with the levelling telescope and noted together with the position of the linear feedthroughs measured by the Heidenhain encoders in order to have a reference value for the angles.

8. After the vacuum chamber is pumped down, the chamber yaw is adjusted such that the beam of a small diode laser mounted near the STXM centered over the optical axis is reflected back into itself by the mirror on the monochromator top flange.

9. The height of the pumped down chamber is controlled with the levelling telescope.

10. The monochromator mirror is moved out of the beam and the direct undulator beam is adjusted to the optical axis at the beam monitor by adjusting the first switching mirror

11. The monochromator mirror is moved in the beam path and the angles are set to the appropriate angles for the energy of the first harmonic of the undulator radiation. If the optics are aligned properly, the fan of the diffracted 
light visible at the beam monitor does not deviate horizontally from the optical axis even if the energy is changed.

\subsection{Characterization of the Monochromator}

The monochromator was installed at BESSY II in spring 2002. After a modification of the spindles for the mirror translation, all rotational and translational movements of the mirror and the grating are functioning as specified.

At an energy of $290 \mathrm{eV}$, the lateral position of the fan of light from the grating deviates by only $1.5 \mathrm{~mm}$ from the direct beam at the beam monitor. This deviation is corrected by adjusting the first switching mirror. With the monochromator mirror fixed, the horizontal beam position shows a dependence on the grating angle of about $4 \mathrm{~mm}$ for an energy change from $275 \mathrm{eV}$ to $480 \mathrm{eV}$ corresponding to a grating exit angle $\beta$ of $1.0^{\circ}$ and $3.9^{\circ}$, respectively. This indicates a yaw of the grating which cannot be corrected without opening the vacuum chamber implying a shut down of the whole microscopy beamline for about two weeks for pumping and bakeout for each adjustment. Therefore, a provision for adjustment of the grating from outside of the vacuum chamber would be desirable. But even in the current state of the adjustment, spectra over a single absorption edge can be obtained without a significant loss of intensity due to a horizontal displacement of the beam.

The motor control of the mirror angle shows an oscillation of the position of the feedthrough of about a micrometer, probably because of play in the feedthrough. Therefore, the mirror control is switched on only to turn the mirror to the angle belonging to a different absorption edge and switched off while acquiring images or spectra. The position of the feedthrough for setting the grating angle is kept constant with an accuracy better than $0.1 \mu \mathrm{m}$, so the spectral resolution is not deteriorated by oscillations around the set position.

Because of the small spectral bandwidth of the first harmonic beam of the undulator U41 (see Sec. 3.1), the undulator gap is adjusted synchronously to the grating movement when acquiring a spectrum. The currently used method of steering the undulator via the BESSY control network leads to a minimum dwell time per energy step of about one second. If shorter dwell times are required, for example to avoid radiation damage to the specimen, the focal spot on the sample has to be kept in motion by acquiring spectra of lines or images (stacks) instead of point spectra. If spectra of a small energy interval up to about $5 \mathrm{eV}$ are to be recorded, the undulator can be set to a constant medium gap to acquire spectra with shorter dwell times by adjusting only the grating angle. This makes dwell times of order milliseconds possible if the grating movement and the intensity read out are properly synchronized. 


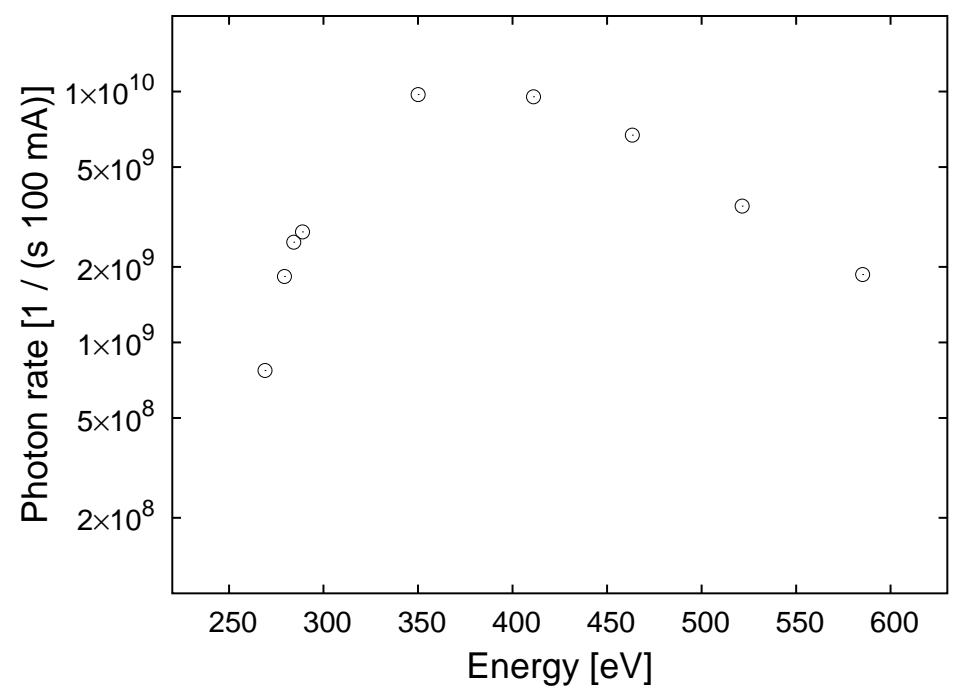

Figure 3.14: Measured spatially coherent photon rate illuminating the zone plate. The rate has been measured with a photo diode at the beam monitor and normalized to a beam current of $100 \mathrm{~mA}$. For each energy step, the undulator gap is set for maximum rate. The mirror angle remained constant at the nominal value for the carbon absorption edge $\gamma=3.479$.

\subsubsection{Photon Rate}

The absolute photon rate illuminating the zone plate in the water window energy range was measured with a photo diode (Fig. 3.14). With the slit apertures of the beam monitor, a $1 \mathrm{~mm}$ wide and $200 \mu \mathrm{m}$ high part of the beam was selected; the rate is scaled to the area of the zone plate. Because of a seized up spindle which blocked the mirror translation, the mirror angle remained constant during this measurement.

Fig. 3.15 shows the rate in the focal spot measured with the pn-CCD detector around the carbon absorption edge and corrected for the pn-CCD quantum detection efficiency (see Fig. 5.12) and the vacuum window transmission. For this measurement, the mirror angle was set to $\gamma=3.55^{\circ}$, where the intensity is by a factor of five higher than at the nominal $\gamma=3.479^{\circ}$. Taking into account the diffraction efficiency of the zone plate and the transmissions of the zone plate and vacuum window silicon membranes, the intensity in the focal spot can be expected to be $1 / 200$ to $1 / 100$ of the intensity illuminating the zone plate. The maximum rate of about $90 \mathrm{MHz}$ decreases by up to two orders of magnitude at $286 \mathrm{eV}$ and $292 \mathrm{eV}$. This is due to carbonaceous contamination on the beamline optics. Additionally, the detector is partly covered with organic debris after the vacuum vessel was vented with the cooled down detector after a vacuum window broke. Part of the debris could be removed by flushing with acetone and isopropyl alcohol. 


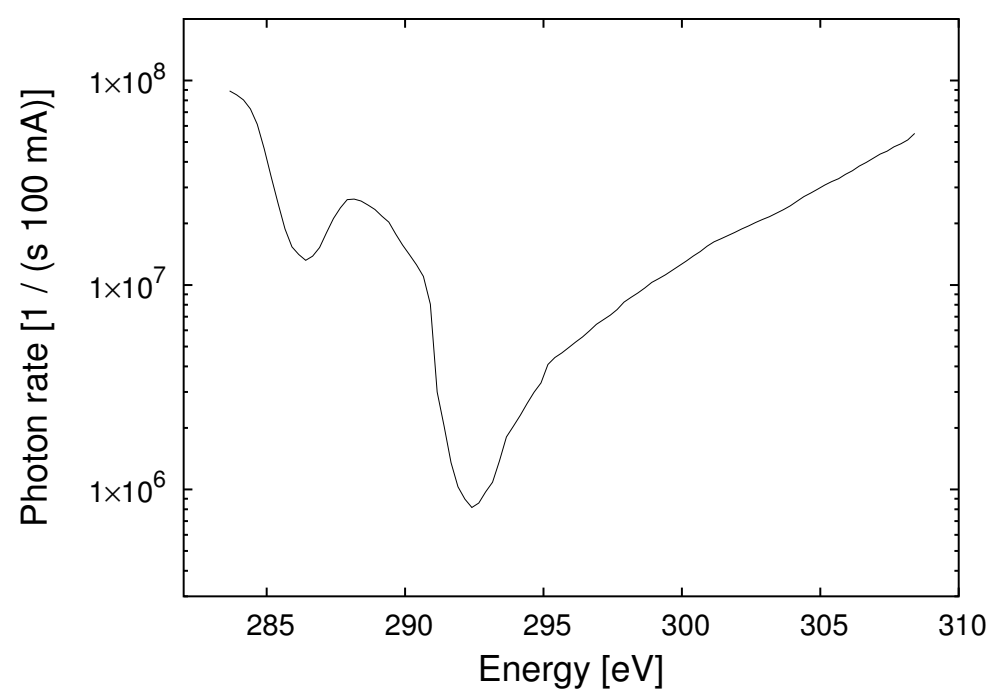

Figure 3.15: Measured photon rate in the focal spot at the carbon absorption edge normalized to a beam current of $100 \mathrm{~mA}$ [84]. The photons are detected with the pnCCD detector without active anode reset (see Sec. 5.4.2). The undulator gap is adjusted synchronously to the grating movement for maximum rate at each energy. The mirror angle is constant $\left(\gamma=3.55^{\circ}\right)$, while the grating is turned to change the selected energy.

The minimum photon numbers required for a signal-to-noise ratio of 3 calculated for the two example specimens in Sec. 1.4.3 can be used to estimate the minimum dwell time per pixel. To image structures with a spatial frequency of $10 \mathrm{\mu m}^{-1}$ in specimen $\mathrm{A}$, a montmorillonite grating in a water layer, $10^{6}$ photons per pixel in the focus are needed. The photon rate in the focal spot at $284 \mathrm{eV}$ is $80 \mathrm{MHz}$, so the minimum pixel dwell time is $13 \mathrm{~ms}$. Specimen B, a carbon grating embedded in a $500 \mathrm{~nm}$ thick montmorillonite matrix in water imaged with elemental contrast at the carbon absorption edge, needs a photon number of $2 \times 10^{6}$ to image structures with $10 \mathrm{\mu m}^{-1}$ spatial frequency. This corresponds to a dwell time of about $40 \mathrm{~ms}$ if the specimen is imaged at $284 \mathrm{eV}$ and $308 \mathrm{eV}$.

Because of the high absorption and the low contrast at high spatial frequencies, these example specimens need to be illuminated with rather high photon numbers; if a lower resolution suffices or and dry samples without the absorption of the water layer and the second silicon nitride membrane are imaged, much lower photon numbers and therefore shorter dwell times are required. If no spectral information is needed, the samples can also be imaged near the oxygen absorption edge, where the transmission of the water layer and the silicon and silicon nitride membranes is by more than one order of magnitude higher while the contrast is similar to that at the carbon absorption edge. 


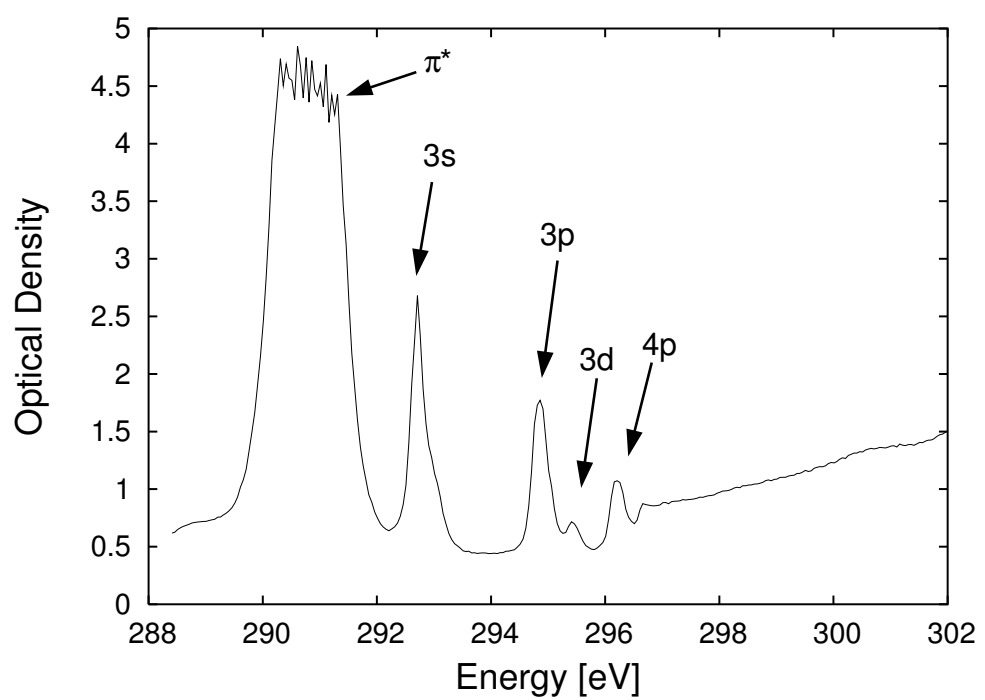

Figure 3.16: NEXAFS spectrum of $505 \mu \mathrm{m} \mathrm{CO}_{2}$ at atmospheric pressure at the carbon $\mathrm{K}$ absorption edge. The peaks belonging to the $\pi^{*}$ and to some of the Rydberg transitions are indicated. The $\pi^{*}$ absorption peak is saturated because of higher order spectral contamination (see Sec. 3.5.4)

\subsubsection{Measurement of the Spectral Resolution}

The spectral resolution of the monochromator is measured using NEXAFS spectra with well-known spectral features following the approach described by Smith et al. [77]. To determine the spectral resolution at the carbon absorption edge, carbon dioxide is led through the capillary of a modified sample holder into the air gap between the OSA and detector vacuum windows. Fig. 3.16 shows the spectrum obtained with a gap of $505 \mu \mathrm{m}$ filled with carbon dioxide.

By fitting Gaussian distributions to the absorption peaks, a full width at half maximum of $254 \mathrm{meV}$ has been determined for the 3d line. In Ref. 42, this line has a width of $146 \mathrm{meV} \pm 20 \mathrm{meV}$. By quadratically subtracting the energy resolution given in Ref. 42, assuming the line may be approximated by a Gaussian distribution, a natural line width of $142 \mathrm{meV} \pm 20 \mathrm{meV}$ is obtained. Hence, the energy resolution of the STXM monochromator at $296 \mathrm{eV}$ is $\Delta E=$ $211 \mathrm{meV} \pm 14 \mathrm{meV}$ corresponding to a monochromaticity of $E / \Delta E=1400 \pm 90$.

A possible reason for the deviation from the theoretical estimate for the monochromaticity of about 3500 (see Fig. 3.7) is that the ray tracing calculations do not take into account the length of the undulator leading to an overstated monochromaticity. Furthermore, the grating surface could be deformed through heat, if the cooling of the grating is non-uniform. The monochromaticity depends strongly on the surface tangent error of the grating [21]. Up to now, the mirror angle was kept constant at the nominal value for $283 \mathrm{eV}$. By varying the mirror angle, the monochromaticity could possibly be improved if the actual mirror 
angle deviates from the nominal value.

\subsubsection{Energy Reproducibility}

To measure the reproducibility of the energy set with the monochromator, multiple NEXAFS spectra of $\mathrm{CO}_{2}$ were acquired. The absorption resonances in the spectra were found to be at constant energies except for an initial drift of $0.1 \mathrm{eV}$ shortly after the beam shutter was first opened. This may be explained by thermal drift of the beamline optics reaching its steady state after a certain time. The energy scale has to be re-calibrated only if the position of the X-ray beam changes because of variations of the electron beam orbit in the storage ring.

\subsubsection{Measurement of Higher Harmonics Radiation}

The amount of spectral contamination by higher harmonics radiation (see Sec. 3.2.3) has been measured in two different ways: the optical density of the $\pi^{*}$ line in the $\mathrm{CO}_{2}$ NEXAFS spectrum where saturation occurs and the measurement of the intensity when the monochromator is set to the double energy.

In the carbon dioxide spectrum in Fig. 3.16, the $\pi^{*}$ peak at $290.7 \mathrm{eV}$ is saturated at an optical density of 4.5. The saturation arises from radiation of higher energies being transmitted through the sample even when the absorption at the first harmonic energy is very high. The amount of detected higher harmonics radiation may be estimated from the saturation optical density. An optical density of 4.5 corresponds to a higher order spectral contamination of the measured signal of $\exp (-4.5)=1.1 \%$.

To estimate the amount of spectral contamination by the second harmonic at an energy of $310 \mathrm{eV}$, the relative efficiency of the beamline and the zone plate for the second harmonic radiation is measured by comparing the intensity with the first harmonic of the undulator at $310 \mathrm{eV}$ to the intensity measured with the first harmonic at $620 \mathrm{eV}$ (Fig. 3.17). The measured intensity ratio of $1 / 228$ has to be multiplied with the ratio of the direct beam intensity at an energy of $620 \mathrm{eV}$ in the second and first harmonic, for which a value of $1 / 9$ was calculated with SPECTRA. Hence, the total suppression of the measured second harmonic signal at a photon energy of $310 \mathrm{eV}$ is about $5 \times 10^{-4}$. Note that the relative amount of the second order radiation in Fig. 3.17 increases with the distance from the undulator peak energy. Hence, a reduction of the photon rate by adjusting the undulator gap so that an off-peak energy is selected leads to higher second order contamination.

The discrepancy with the relative spectral contamination of $1.1 \%$ obtained from the saturation of the $\pi^{*}$ absorption peak is attributable to the carbon contamination of the beamline optics. At a photon energy of $290.7 \mathrm{eV}$, the photon rate is one order of magnitude lower than at $310 \mathrm{eV}$ (see Fig. 3.15). The higher 


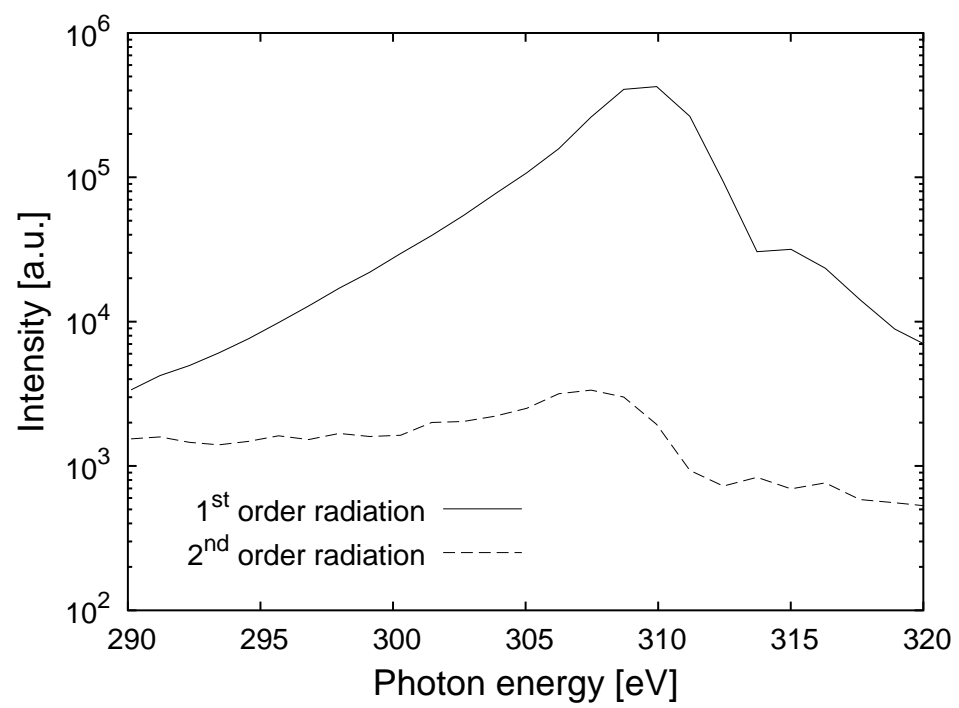

Figure 3.17: Measurement of the second order spectral contamination. The intensity is measured with the undulator gap set corresponding to an energy of $310 \mathrm{eV}$ and $620 \mathrm{eV}$ to estimate the beamline efficiency for the second compared to the first order radiation (see text). The energy scale applies to the energy set with the monochromator.

order radiation is affected much less by the carbon contamination, so the relative spectral contamination is higher at the $\pi^{*}$ peak.

\subsection{Summary}

Because of the small source size of the undulator and the large distance to the STXM, the zone plate is illuminated by the direct beam spatially coherently without restricting the beam diameter with a pinhole. Therefore, the monochromator can be designed with the minimum number of optics: it consists only of a plane mirror and a plane varied line spacing grating without entrance or exit slits. The main advantages of this concept are practicability and high flux throughput.

The monochromator is very easy to align, because the vertical fan of light from the grating has to be adjusted in only one dimension to impinge on the zone plate centrally. However, the adjustment of the grating yaw could be improved by making it possible to change the yaw from the outside of the vacuum vessel.

In the whole water window energy range, the coherent photon rate is sufficient to acquire STXM images of low-contrast hydrated specimens with reasonable dwell times. Around the carbon edge, a photon rate in the focal spot of $1 \mathrm{MHz}$ to $90 \mathrm{MHz}$ normalized to $100 \mathrm{~mA}$ beam current has been measured. The rate decreases sharply at $286 \mathrm{eV}$ and $292 \mathrm{eV}$ because of carbonaceous contamination of the beamline optics. This could be reduced by cleaning the optics surfaces as described in Ref. 17. The peak photon rate is reached at about $400 \mathrm{eV}$ near the 
nitrogen absorption edge. The photon rate depends strongly on the deflection angle set with the mirror. Further experiments are necessary to find the mirror angle giving the optimal compromise between photon rate and spectral resolution.

A monochromaticity of 1400 has been measured at the carbon absorption edge using the sharp Rydberg transitions in the $\mathrm{CO}_{2}$ NEXAFS spectrum. While a higher monochromaticity has been expected according to ray tracing calculations, this monochromaticity is high enough to obtain NEXAFS spectra of good quality.

Radiation of higher undulator harmonics is efficiently suppressed by the choice of the mirror coating. At $290.7 \mathrm{eV}$, a higher energy spectral contamination of $1.1 \%$ has been measured. The relative intensity of the second harmonic at $310 \mathrm{eV}$ has been measured to be $0.05 \%$. Therefore, quantitative measurements at the carbon edge are possible for samples with optical densities up to about 4 . 


\section{Chapter 4}

\section{The Object Stage}

In scanning transmission X-ray microscopes, piezoelectric actuators are used for the high-resolution scan to reach the diffraction-limited resolution of the zone plate. Additionally, the sample can be positioned coarsely with DC or stepper motors. In contrast to most existing STXMs, where the beam from the monochromator overfills the zone plate by only a small amount, the monochromator of the BESSY II STXM without entrance or exit slits illuminates a large area in the zone plate plane homogeneously (see Sec. 3.2.1). Therefore, it is possible to move the zone plate instead of the sample with the piezo stage for the high resolution scan, so the assembly for the high resolution scan can be separated from that of the coarse scan. Because of the separation of the high and low resolution positioning stages, the mechanical setup can be built more rigid compared to a setup with the piezo stage mounted on top of an X-Y-Z stage for the coarse specimen movement and focusing. In the following, the whole setup for the zone plate, OSA and sample movement is referred to as the object stage.

To keep the mechanical design simple, the OSA is not scanned together with the zone plate during the high resolution scan. This setup limits the maximum image field for which the OSA still keeps light of unwanted diffraction orders from reaching the sample. In the next section, the maximum image diameter is calculated as a function of OSA position and diameter.

\subsection{Image Field Limitation by the OSA and the Central Stop}

Fig. 4.1 shows a schematic ray diagram of the zone plate, the OSA and the sample for imaging in the first and in the third diffraction order. For imaging in the first diffraction order, three conditions must be fulfilled:

1. No zero order radiation passes the OSA:

$$
D_{\mathrm{Img}} \leq D_{\mathrm{CS}}-D_{\mathrm{OSA}}
$$



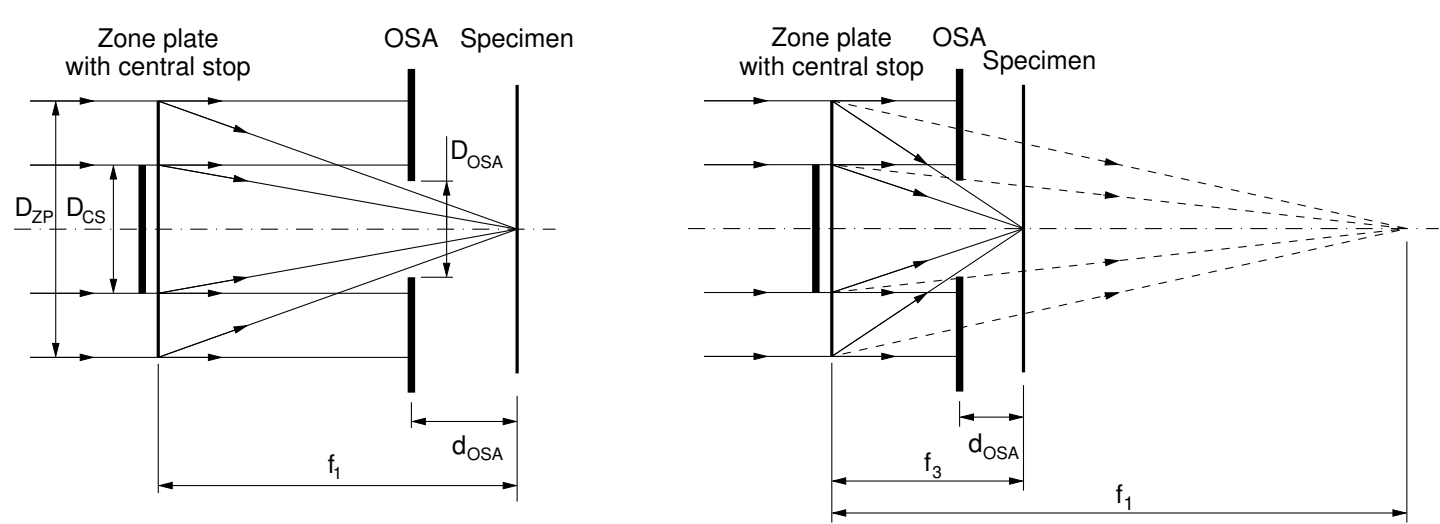

Figure 4.1: Schematic STXM ray diagram for imaging in the first (left) and in the third diffraction order (right). Diffraction orders higher than the one used for imaging are not shown. $D_{\mathrm{ZP}}$ is the zone plate diameter, $D_{\mathrm{CS}}$ is the central stop diameter, and $D_{\mathrm{OSA}}$ is the OSA diameter. The first and third diffraction order focal lengths are $f_{1}$ and $f_{3}$, the distance of the OSA from the specimen is $d_{\mathrm{OSA}}$.

2. The whole hollow cone of the first order radiation passes through the OSA:

$$
D_{\mathrm{Img}} \leq D_{\mathrm{OSA}}-\frac{d_{\mathrm{OSA}} D_{\mathrm{ZP}}}{f_{1}}
$$

3. The third order radiation is masked out by the OSA:

$$
D_{\mathrm{Img}} \leq 2 D_{\mathrm{CS}}-\frac{d_{\mathrm{OSA}} D_{\mathrm{CS}}}{f_{3}}-D_{\mathrm{OSA}}
$$

For imaging in the third diffraction order, Eqs. (4.1) and (4.2) hold with the third order focus $f_{3}$ instead of $f_{1}$. In addition, the hollow cone of the first diffraction order, which is one order of magnitude brighter (see Eq. (1.12)), must be completely masked out by the OSA:

$$
\begin{aligned}
& D_{\mathrm{Img}} \leq D_{\mathrm{CS}}-D_{\mathrm{OSA}} \\
& D_{\mathrm{Img}} \leq D_{\mathrm{OSA}}-\frac{d_{\mathrm{OSA}} D_{\mathrm{ZP}}}{f_{3}}, \\
& D_{\mathrm{Img}} \leq \frac{\left(d_{\mathrm{OSA}}+f_{1}-f_{3}\right) D_{\mathrm{CS}}}{f_{1}}-D_{\mathrm{OSA}} .
\end{aligned}
$$

Because the OSA distance $d_{\mathrm{OSA}}$ is always less than the focal length $f_{3}$, Eq. (4.6) implies Eq. (4.4). Light of the fifth and higher orders is not taken into account here; the detector pinhole must be placed such that it keeps them from reaching the detector.

Fig. 4.2 shows the maximum image diameter as a function of OSA distance for several OSA diameters for imaging in the first and third diffraction order with the zone plate currently used for the BESSY II STXM. An OSA diameter 

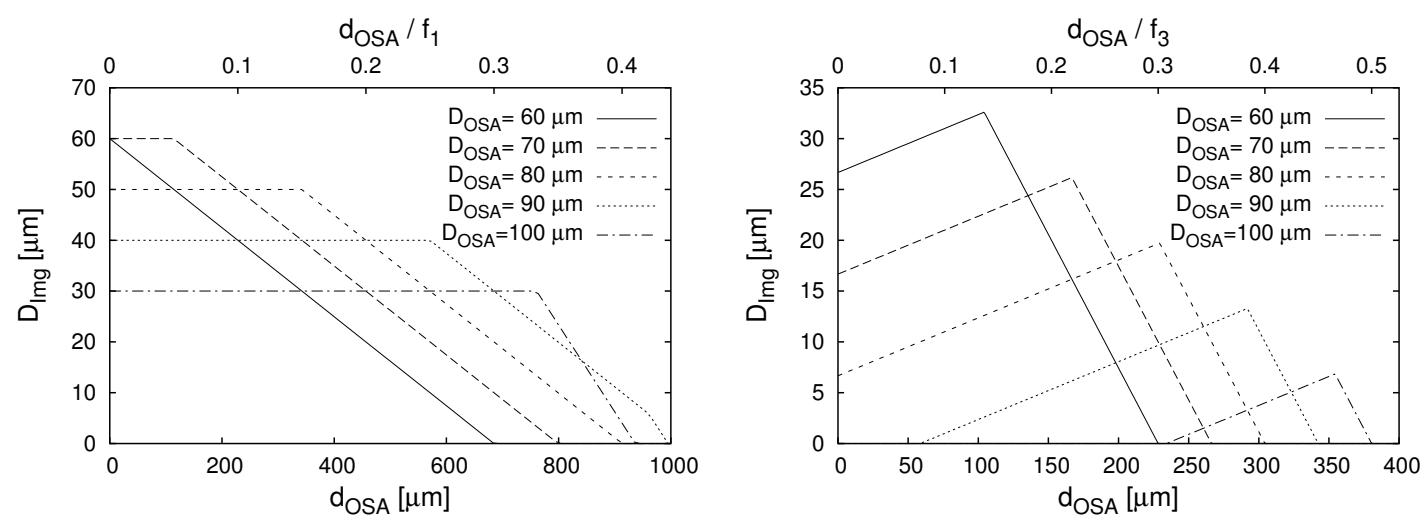

Figure 4.2: Maximum image diameter $D_{\mathrm{Img}}$ as a function of the distance $d_{\mathrm{OSA}}$ of the OSA from the specimen for several OSA diameters $D_{\mathrm{OSA}}$. Left: maximum $d_{\mathrm{Img}}$ for the first diffraction order as determined by Eqs. (4.1) to (4.3). Right: maximum $d_{\text {Img }}$ for the third diffraction order as determined by Eqs. (4.4) to (4.6). The lower abscissa scales apply for focal lengths of $f_{1}=2283 \mu \mathrm{m}$ and $f_{3}=761 \mu \mathrm{m}$ corresponding to a photon energy of $283 \mathrm{eV}$. The image diameters are calculated for the zone plates currently used in the STXM (see Tab. 4.1)

of $80 \mu \mathrm{m}$ is a good compromise between image diameter and OSA distance. In the first order, images with a diameter of up to $50 \mu \mathrm{m}$ are possible for an OSA distance of $350 \mu \mathrm{m}$ at $283 \mathrm{eV}$. In the third order, images of $20 \mu \mathrm{m}$ diameter can be obtained with an OSA distance of $230 \mu \mathrm{m}$. Because hydrated specimens are held between two silicon nitride membranes with $200 \mu \mathrm{m}$ support frame thickness (see Sec. 4.5), this would mean a distance between the OSA and the support frame of only $30 \mu \mathrm{m}$. Therefore, membranes with thinned support frames have to be used for third order imaging of hydrated specimens at the carbon absorption edge. Alternatively, dry specimens may be prepared on a single silicon nitride membrane with the support frames oriented to the detector side to obtain a larger distance. If no carbon sensitivity is needed, third order images can be taken at the oxygen absorption edge with larger OSA distances.

\subsection{Zone Plates for the STXM}

The nickel phase zone plates currently used for the BESSY II STXM have been built by S. Rehbein and D. Weinrich at the Institute for X-Ray Physics [62]. The zone plate parameters are summarized in Tab. 4.1. The zone height is optimized for maximum first-order diffraction efficiency at the carbon absorption edge. Because the electron beam current of the lithography system could not be set to a sufficiently small value at the time of the fabrication of the zone plates, they have a relatively large outermost zone width of $\mathrm{d} r_{N}=50 \mathrm{~nm}$. A large central stop diameter of $D_{\mathrm{CS}}=130 \mathrm{~nm}$ has been chosen to obtain large image fields for the high resolution scan (see Sec. 4.1) at the expense of lower contrast transfer 
Table 4.1: Parameters of the nickel zone plates currently used in the STXM for the first and third diffraction order $m$. The theoretical diffraction efficiency is calculated with scalar diffraction theory not taking into account the absorption of the support membrane.

\begin{tabular}{|c|c|c|c|c|}
\hline & & & \multicolumn{2}{|c|}{$m$} \\
\hline & & & 1 & 3 \\
\hline Outermost zone width & $\mathrm{d} r_{N}$ & $\mathrm{~nm}$ & \multicolumn{2}{|c|}{50} \\
\hline Radius & $r_{N}$ & $\mu \mathrm{m}$ & \multicolumn{2}{|c|}{100} \\
\hline Zone number & $N$ & & \multicolumn{2}{|c|}{1000} \\
\hline Zone height & $h_{\mathrm{z}}$ & $\mu \mathrm{m}$ & \multicolumn{2}{|c|}{130} \\
\hline Central stop radius & $r_{\mathrm{CS}}$ & $\mu \mathrm{m}$ & \multicolumn{2}{|c|}{65} \\
\hline Central stop height & $h_{\mathrm{CS}}$ & $\mu \mathrm{m}$ & \multicolumn{2}{|c|}{550} \\
\hline \multirow[t]{2}{*}{ Focal length } & $f_{m}(283 \mathrm{eV})$ & $\mu \mathrm{m}$ & 2283 & 761 \\
\hline & $f_{m}(524.5 \mathrm{eV})$ & $\mu \mathrm{m}$ & 4231 & 1410 \\
\hline \multirow[t]{2}{*}{ Theor. diff. efficiency } & $\eta_{m}(283 \mathrm{eV})$ & $\%$ & 16.1 & 1.8 \\
\hline & $\eta_{m}(524.5 \mathrm{eV})$ & $\%$ & 13.9 & 1.5 \\
\hline
\end{tabular}
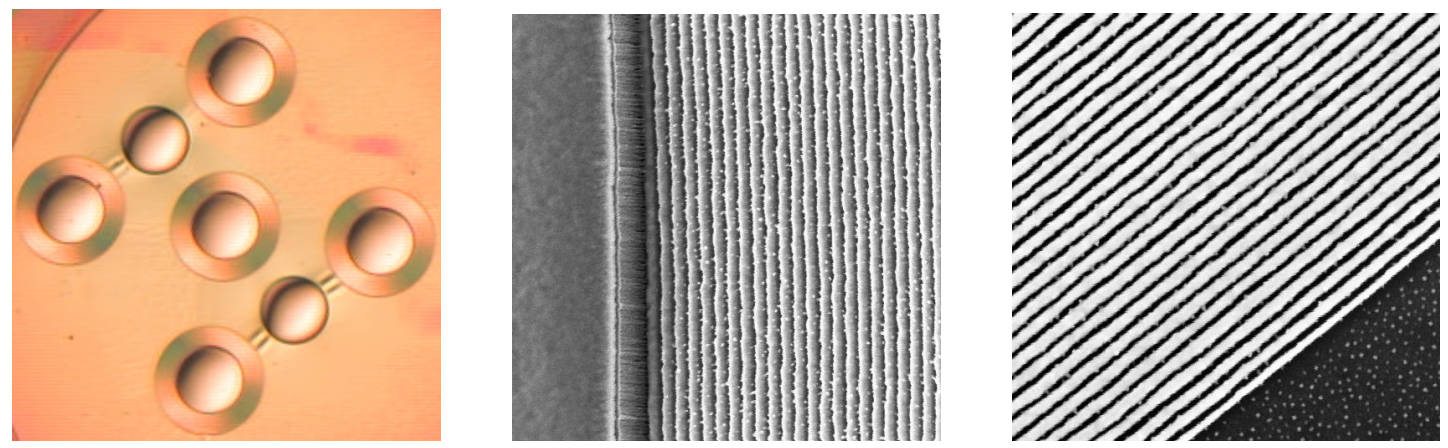

Figure 4.3: Left: differential interference contrast image of a set of five zone plates on a silicon membrane [61]. Center: SEM micrograph of inner zones and central stop (sample tilted by $45^{\circ}$ ) [62]. Right: SEM micrograph of outermost zones [62].

at high spatial frequencies (see Sec. 1.4).

The zone plates are fabricated as a set of five on a $130 \mathrm{~nm}$ thick silicon support membrane (see Fig. 4.3). The circular silicon membrane is $1 \mathrm{~mm}$ across; it is etched into a $200 \mu \mathrm{m}$ thick silicon wafer with a diameter of $4.8 \mathrm{~mm}$ by an anisotropic etching process $[44,56]$ such that an ring-shaped support frame remains. Fig. 4.4 shows the zone plate nanostructuring process. The PMMA resist is exposed with the zone pattern in a Leica LION LV1 lithography system. The pattern is transfered into an intermediate silicon mask by reactive ion etching 

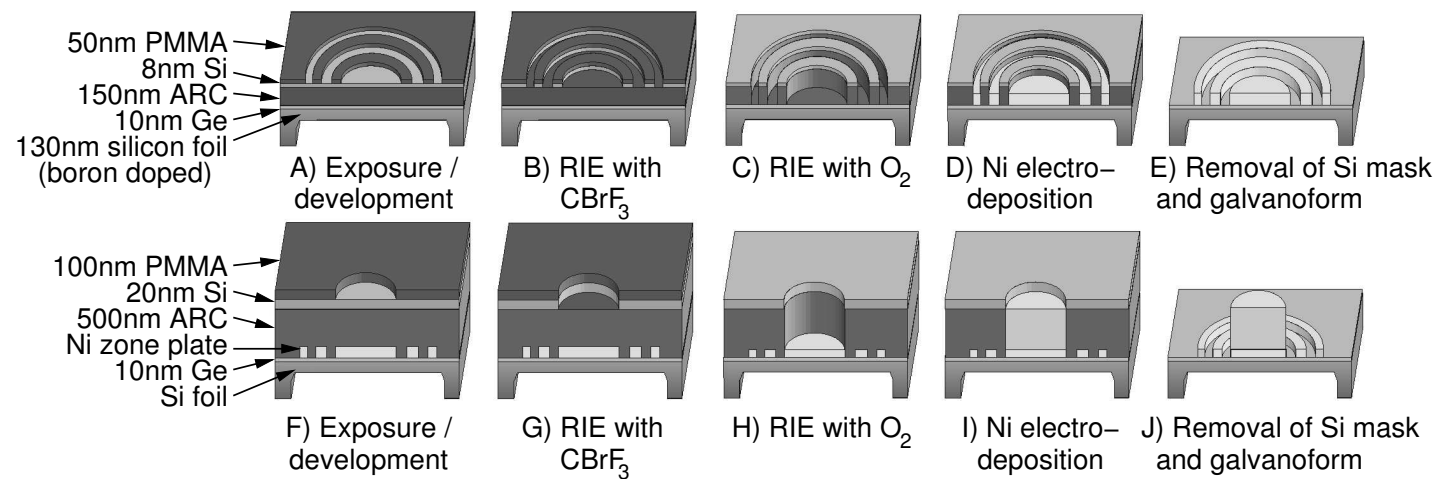

Figure 4.4: Nanostructuring steps for the fabrication of the zone structures (top) and the central stop (bottom) of the STXM zone plates [62]. See text.

(RIE). Using the silicon mask, the pattern is etched by RIE into a $150 \mathrm{~nm}$ thick polymer layer serving as a galvanoform. After electrodeposition of the nickel zones, the mask and the galvanoform are removed leaving the finished zones. This process is repeated to build the nickel central stop on top of the zone plate. Several of the zone plates built have been found to show astigmatism (see Sec. 6.1). This is attributable to a tilted position of the zone plate substrate in the sample holder during the electron beam exposure of the zone pattern.

\subsection{Mechanical Setup of the Object Stage}

The object stage comprises three different translation stages for the zone plate, the OSA and the sample movement. In the following, a coordinate system is used where the $\mathrm{Z}$ axis is parallel to the optical axis and the $\mathrm{X}$ and $\mathrm{Y}$ axes are the horizontal and vertical axes in the specimen plane.

The zone plate is scanned with nanometer-precision in $\mathrm{X}$ and $\mathrm{Y}$ by a piezoelectric flexure stage with integrated capacitance micrometers, which is described in detail in Sec. 4.4. If the pitch or yaw of the moving platform of the stage vary during the scanning motion, the zone plate position deviates from the position measured in the plane of the stage. To minimize this so-called Abbe error, the zone plate holder has to protrude as little as possible from the piezo stage. Furthermore, the minimum pixel dwell time is proportional to the distance of the detector from the sample (see Sec. 5.2.1). Therefore, the object stage is designed for minimum distance between the piezo stage and the detector. Tab. 4.2 summarizes the movements of the object stage components and of the detector pinhole. Except for the sample, all components can be moved in all three directions. Therefore, any relative position of the zone plate, the OSA, the specimen and the detector pinhole can be set within their respective ranges.

Fig. 4.5 shows the mechanical setup of the object stage. The zone plate mounted on the zone plate holder (a) (see Sec. B.1) and the piezo stage (b) are 


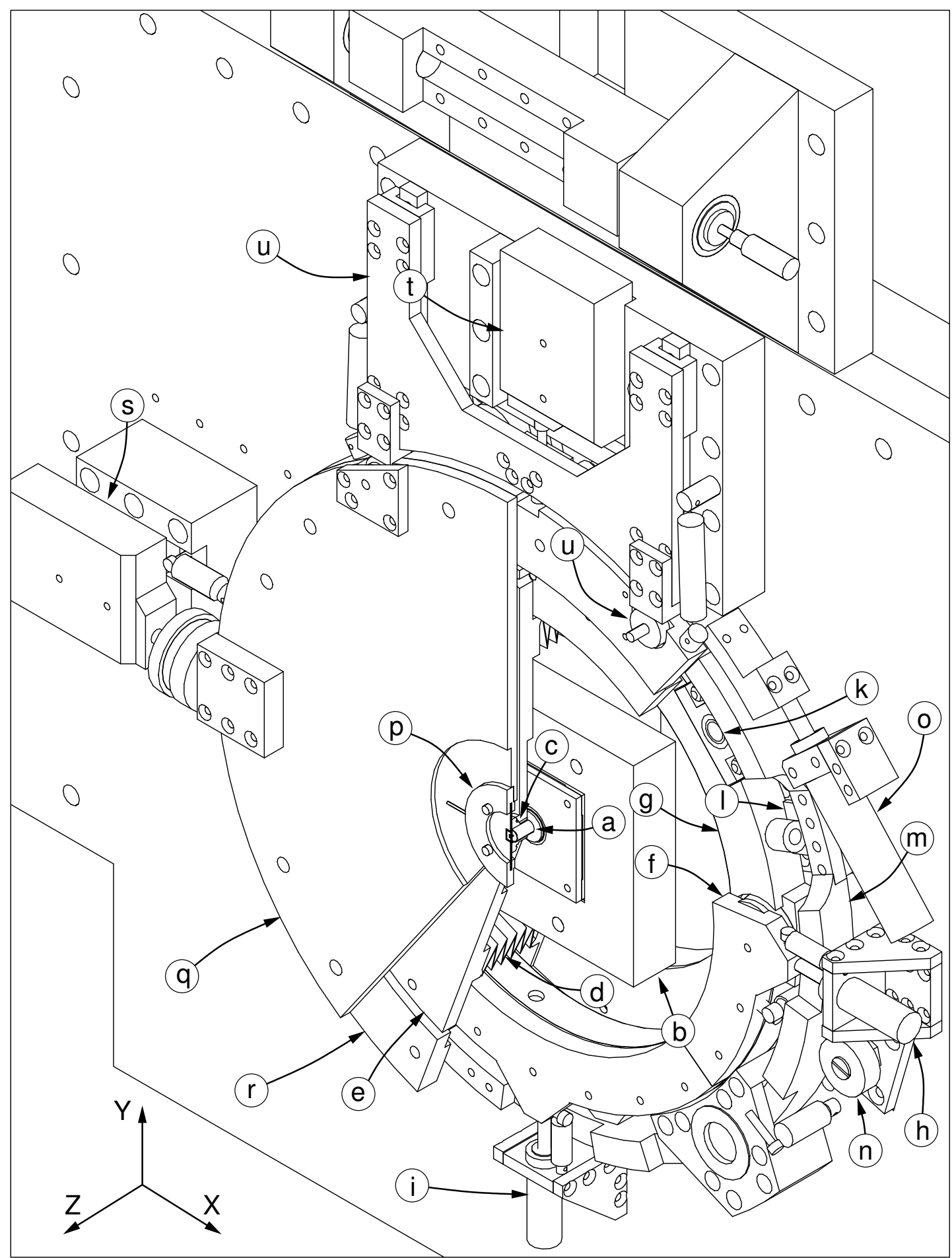

Figure 4.5: Cut-away view of the object stage (see text). The X-ray beam comes in from the right. The visible light microscope is not shown. See Fig. 4.6 for a view of the zone plate $\mathrm{Z}$ drive from the upstream side. 
Table 4.2: Operation of the different movements of the object stage components and of the detector pinhole, which is moved together with the detector vacuum vessel (see Fig. 5.5).

\begin{tabular}{lccc}
\hline & \multicolumn{3}{c}{ Direction } \\
Component & $\mathrm{X}$ & $\mathrm{Y}$ & $\mathrm{Z}$ \\
\hline Zone plate & Piezo & Piezo & Motor \\
OSA & Manual & Manual & Manual \\
Sample & Motor & Motor & \\
Visible light microscope & Manual & Manual & Motor \\
Detector & Manual & Manual & Motor \\
\hline
\end{tabular}

located in a vacuum chamber directly connected to the beamline. To adjust the zone plate $\mathrm{Z}$ position for focusing, it is moved by two flexible steel rods in a slide bearing as described in Fig. 4.6. A slide bearing was chosen over a rotary stroke bearing to minimize the lateral deviation during the focusing motion caused by imperfect balls which limits the ability to obtain spectra from small sample features.

The OSA (c) (see App. B.2) has to be adjustable over the center of each of the five zone plates on the silicon membrane giving rise to a minimum X-Y translational range of $600 \mu \mathrm{m}$, which exceeds the range of the piezo stage. Because the vacuum exit window is held by the OSA, the OSA has to be connected to the vacuum chamber with a bellow to be able to move it. To keep the distance from the piezo stage to the sample short, the bellow is not placed near the OSA but around the piezo stage $(\mathrm{d})$. The OSA is moved together with the OSA front plate (e), on which the air pressure exerts a force of about $2 \mathrm{kN}$. The OSA front plate is fixed to the upper OSA ring (f) resting on ball bearings on the lower OSA ring (g) and can be adjusted in $\mathrm{X}$ and $\mathrm{Y}$ with micrometer screws (h, i). The lower OSA ring is guided in $\mathrm{X}$ and $\mathrm{Y}$ by three rotary stroke bearings $(\mathrm{k})$ and is pressed by the air pressure against three concentrically arranged wedges (l). The wedges are fixed to the OSA Z ring $(\mathrm{m})$ which is guided by three ball bearings (n). If the OSA Z ring is turned by the micrometer screw (o), the wedges lift the lower OSA ring in the $\mathrm{Z}$ direction, so the OSA $\mathrm{Z}$ position can be adjusted.

The sample is held in a wet specimen chamber ( $p$ ) which also fits into the TXM object stage (see Sec. 4.5). The specimen chamber is fixed to an aluminum plate $(\mathrm{q})$ mounted on a support ring $(\mathrm{r})$. The $\mathrm{X}$ and $\mathrm{Y}$ motion of the sample are driven by linear actuators with DC motors and integrated encoders (Physikinstrumente M-232.17) with a unidirectional repeatability of about $0.1 \mu \mathrm{m}$. They are connected to a C-842 motor controller ISA board. While the linear actuator 


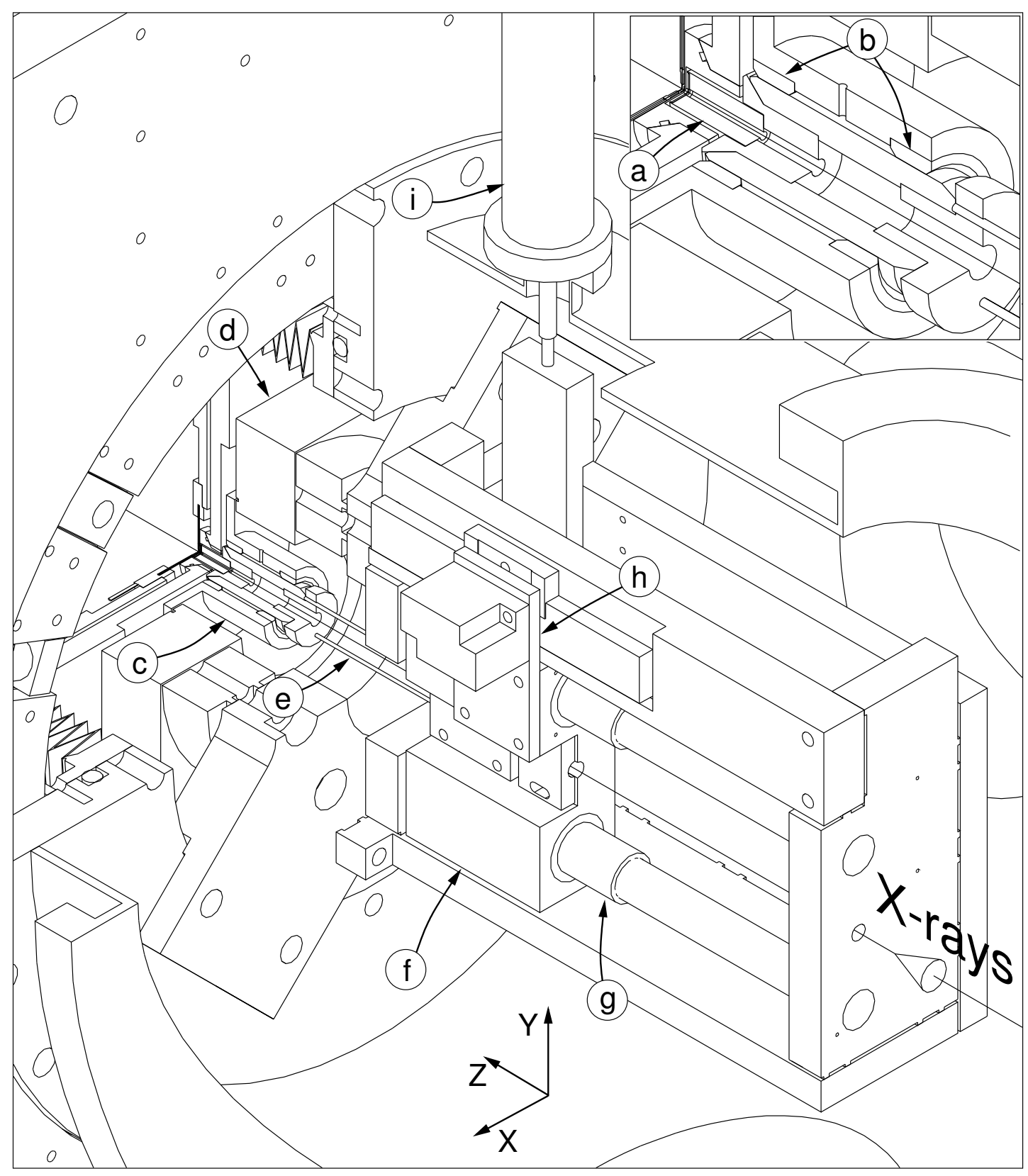

Figure 4.6: Cut-away drawing of the in-vacuum zone plate $\mathrm{Z}$ drive. Inset: magnified view of the zone plate sliding contact bearing. The zone plate holder (a, see Sec. B.1) is screwed into a bored shaft, which is guided by two PEEK sliding contact bearings (b). The sliding contact bearings are held by a part (c) which is moved in X and Y by the piezo stage (d) for the high resolution scan. The shaft is pushed or pulled in $\mathrm{Z}$ direction via two steel rods (e) by a block (f) guided by two linear ball bearings (g). The $\mathrm{Z}$ position of the block is measured with a Heidenhain LIP $481 \mathrm{~V}$ vacuum compatible position encoder (h). The block is moved in $\mathrm{Z}$ direction by an electrical feedthrough (i) via a wedge (not visible). 
for the $\mathrm{X}$ motion (s) pushes directly on the sample holder front plate, the $\mathrm{Y}$ actuator $(\mathrm{t})$ drives a carriage $(\mathrm{u})$ guiding two ball bearings $(\mathrm{v})$ mounted on the front plate to prevent the front plate from turning around the $\mathrm{Z}$ axis but still allowing movement in the $\mathrm{X}$ direction.

The in-vacuum mechanical parts for the zone plate $\mathrm{Z}$ drive (see Fig. 4.6) are made from vacuum compatible aluminum (AlMgSi1) or stainless steel. They are baked out at $180^{\circ}$ before they are assembled. In the zone plate vacuum vessel, a pressure of $5 \times 10^{-8}$ mbar is reached.

\subsubsection{Alignment of the Optical Elements}

The zone plate, the OSA and the detector pinhole are lined up on the optical axis according to the the following procedure:

1. One zone plate of the set of five on the silicon membrane promising to have a good diffraction efficiency and a sufficiently thick central stop is selected. To be able to see the zone plate with the visisble light microscope (VLM), the vacuum vessel has to be vented and the OSA has to be removed.

2. The piezo stage controller is switched on so the zone plate moves to its central position and the VLM cross hair is adjusted to the zone plate center.

3. The $\mathrm{Z}$ positions displayed for the zone plate and the light microscope are set to the same value to obtain a common reference value for the zone plate $\mathrm{Z}$ position measured with the in-vacuum position encoder and for the VLM $\mathrm{Z}$ position.

4. After inserting the OSA and pumping down the vacuum vessel, the OSA is aligned to the light microscope cross hair with the $\mathrm{X}$ and $\mathrm{Y}$ micrometer screws. While pumping down the vacuum vessel, the piezo stage controller has to be switched off to avoid discharges at intermediate air pressures.

5. The light microscope is focused onto the OSA and the $\mathrm{Z}$ position is saved as the OSA position.

6. The light microscope is moved out and the detector vacuum vessel is moved in with a large air gap to get a higher probability of finding the radiation hollow cone with the detector pinhole.

7. The ccdwatch program is started for online display of the frames read out by the pn-CCD detector.

8. The zone plate $\mathrm{Z}$ position is adjusted to the value appropriate for the $\mathrm{X}$ ray energy selected with the monochromator. The $\mathrm{X}$ and $\mathrm{Y}$ position of the detector vessel is scanned until the radiation hollow cone is found and the full ring created by the zone plate with the central spot is visible on 
the detector display without being obstructed by the OSA or the detector pinhole.

9. By turning the BESSY pre-mirror around the vertical axis, the beam impinging on the zone plate is adjusted horizontally such that the measured intensity is maximized.

\subsection{The Zone Plate Scanning Stage}

A Queensgate NPS-XY-100A piezoelectric flexure stage made from Super Invar is employed to position the zone plate. It has integrated capacitance position sensors for operation in closed loop mode. The maximum travel in $\mathrm{X}$ and $\mathrm{Y}$ direction is $100 \mu \mathrm{m}$. For a constant position, the specified position noise is about $0.5 \mathrm{~nm}$ RMS and the maximum linearity error is $0.007 \%$ of the whole travel or $7 \mathrm{~nm}$ [59]. Sec. 4.4.1 describes the measured positioning accuracy of the stage in motion.

The stage is controlled by a 21-bit digital DSP controller (Queensgate NPS $3220)$ with a loop cycle time of $100 \mu \mathrm{s}$. The controller is equipped with two interfaces to connect to the control computer, a standard serial RS232 interface and a parallel PAR-C interface. The serial interface is used to set control parameters like the PID parameters (see Sec. A.2) for the servo loop. Via the parallel interface, position commands can be transmitted to the controller via a custom bus protocol with a rate of up to $4 \mathrm{kHz}$. In the control computer, a National Instruments PC-DIO-24/PnP digital I/O board is used to generate the signals for the parallel interface. A Real Time Linux kernel module controls the interface board with hard real time accuracy (see Sec. A.1).

\subsubsection{Test of the Stage Accuracy in Motion}

Deviations of the stage from the commanded position can degrade the image acquired in two different ways: the repeatability or positioning noise limits the resolution and the signal-to-noise ratio of the image. Non-linear but repeatable deviations appear as distortions in the image and can be corrected. To test the accuracy of the scanning motion, the position is measured independently of the stage's built-in capacitance micrometers using a Heidenhain CP $60 \mathrm{~K}$ position encoder with a signal period of $2 \mu \mathrm{m}$ (see Fig. 4.7). The Heidenhain IK121 ISA interface board employed to record the encoder position interpolates 1024-fold giving rise to a measurement resolution of about $2 \mathrm{~nm}$. A Real Time Linux kernel module reads out the position each millisecond with microsecond accuracy. As an approximation of continuous motion, the pixel dwell time is set to $0.5 \mathrm{~ms}$ with a step size of $12.5 \mathrm{~nm}$ per pixel and 2500 pixels per scan line. If only the central $25 \mu \mathrm{m}$ of the $31.25 \mu \mathrm{m}$ scan range are displayed to avoid distortions generated by 


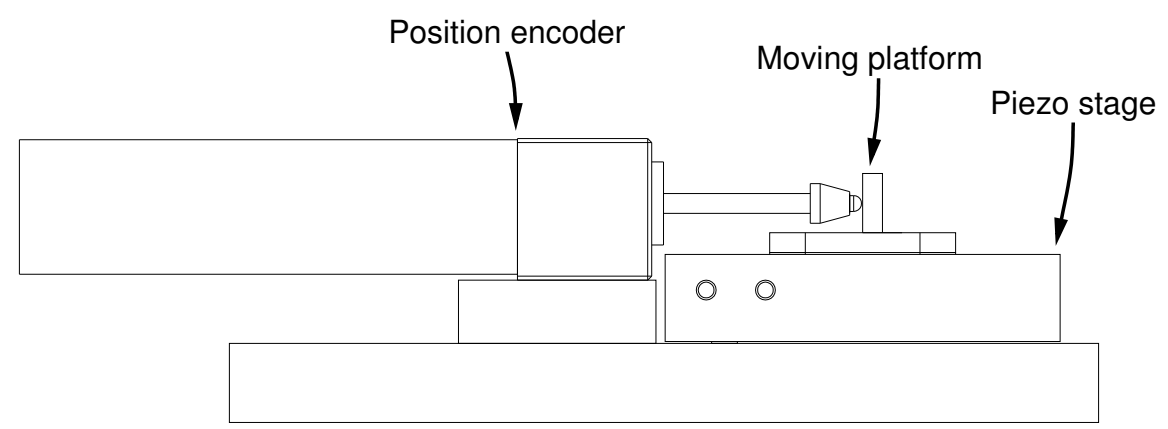

Figure 4.7: Side view of the test setup for measuring the accuracy of the piezo stage motion.
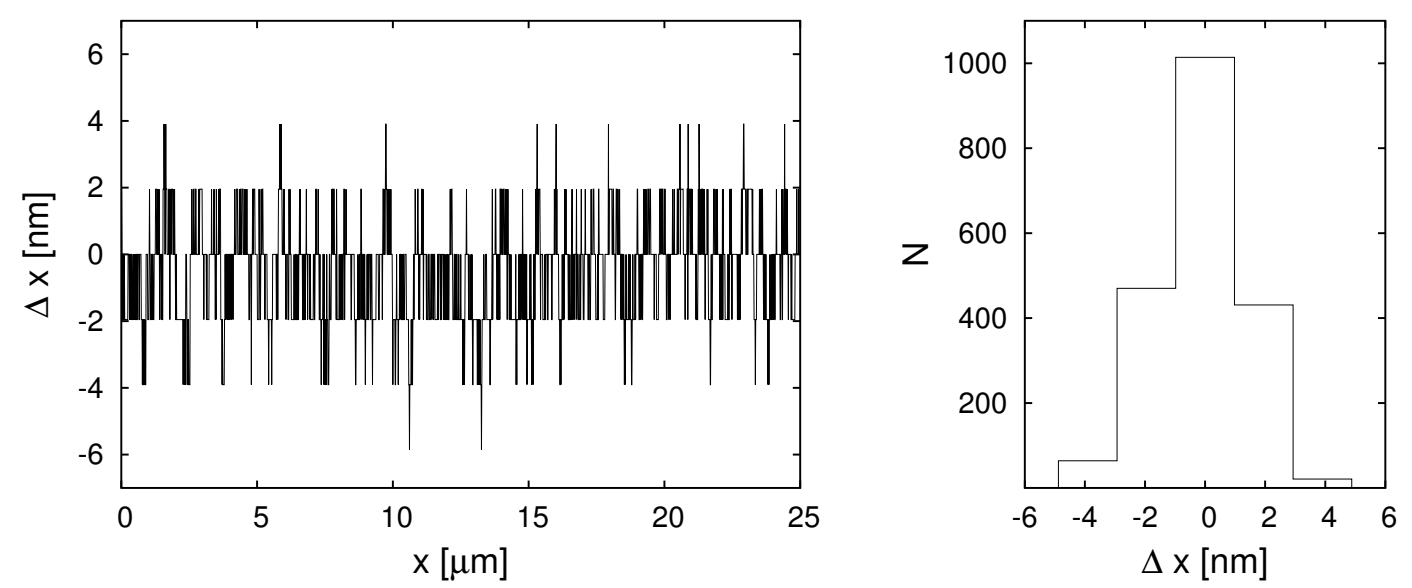

Figure 4.8: Repeatability of the piezo stage for a realistic scan motion (see text) [94]. Shown is the position difference of the positions of two subsequent scan lines measured with an external position encoder with $2 \mathrm{~nm}$ resolution. Left: position deviation as a function of position. Right: histogram of the deviations. The position standard deviation is $1.5 \mathrm{~nm}$.

the return to the line start position, this corresponds to an image with $500 \times 500$ pixels of $50 \times 50 \mathrm{~nm}^{2}$ size with a detector integration time of $2 \mathrm{~ms}$.

According to the calculations shown in Sec. 1.4.2, the standard deviation of the positioning noise of the sample with respect to the zone plate has to be kept below $5 \mathrm{~nm}$ to obtain diffraction-limited resolution with a zone plate of $50 \mathrm{~nm}$ outermost zone width without a significant decrease of the signal-to-noise ratio. Fig. 4.8 shows the repeatability measured as the position difference of two subsequent scan lines. The standard deviation of the measured position differences is $1.5 \mathrm{~nm}$, so the piezo stage is suited even for imaging at resolutions well below $50 \mathrm{~nm}$.

For the measurement of the stage nonlinearity, the position sensor was first calibrated using the built-in capacitance micrometers at constant positions. The deviation of the stage from the commanded position while scanning is shown in Fig. 4.9. Over the $25 \mu \mathrm{m}$ scan range, the measured position deviates by less than 


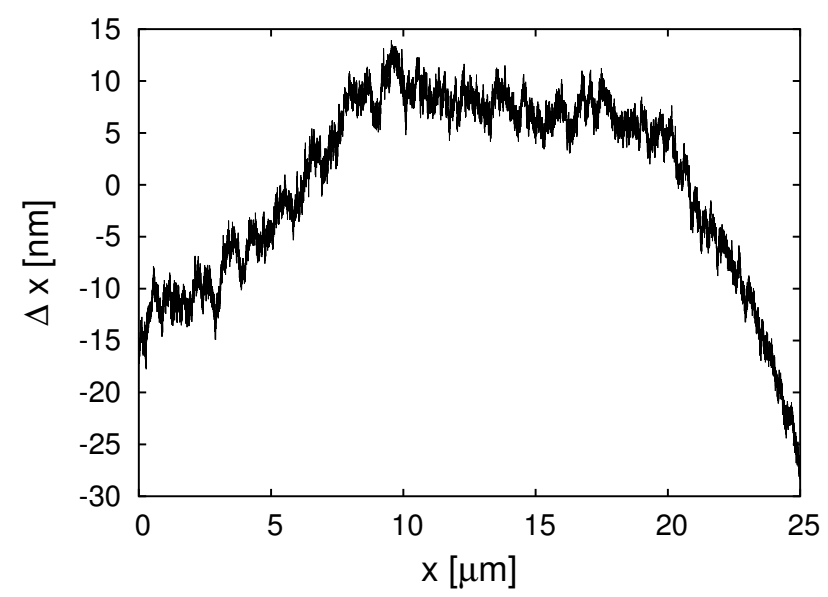

Figure 4.9: Nonlinearity of the piezo stage for a realistic scan motion (see text): Deviation of the position measured with an external position encoder from the commanded position [94].

$30 \mathrm{~nm}$ from the commanded position.

\subsection{The Wet Specimen Chamber}

For imaging hydrated specimens at room temperature, a wet specimen chamber has been developed for the BESSY TXM [52], where the specimen is held between two polyimide foils. The thickness of the water layer can be adjusted with syringes connected to the chamber. Because of the carbon contained in the polyimide foils, a different membrane material has to be employed for spectromicroscopy at the carbon absorption edge, where the majority of the BESSY II STXM experiments will take place.

For carbon spectromicroscopy, it is common to use silicon nitride membranes [51], which are commercially available in various sizes and thicknesses. For the STXM at BESSY II, the TXM wet specimen chamber is used with a modification to be able to use silicon nitride membranes (see Fig. 4.10). The membrane frames are glued onto thin metal sheets, which are held by the two standard TXM wet specimen chamber rings. Since the specimen chamber also fits into the TXM object stage, a sample can be imaged with high spatial resolution in the TXM and then be transferred to the STXM for spectromicroscopic analysis and vice versa.

Dry specimens are prepared on a single membrane glued onto a metal sheet with the foil oriented towards the OSA. Therefore, the specimen can be brought very close to the OSA which is necessary for imaging in the third diffraction order at the carbon absorption edge (see Sec. 4.1).

A special sample holder frame with long capillaries is used to flush the air 


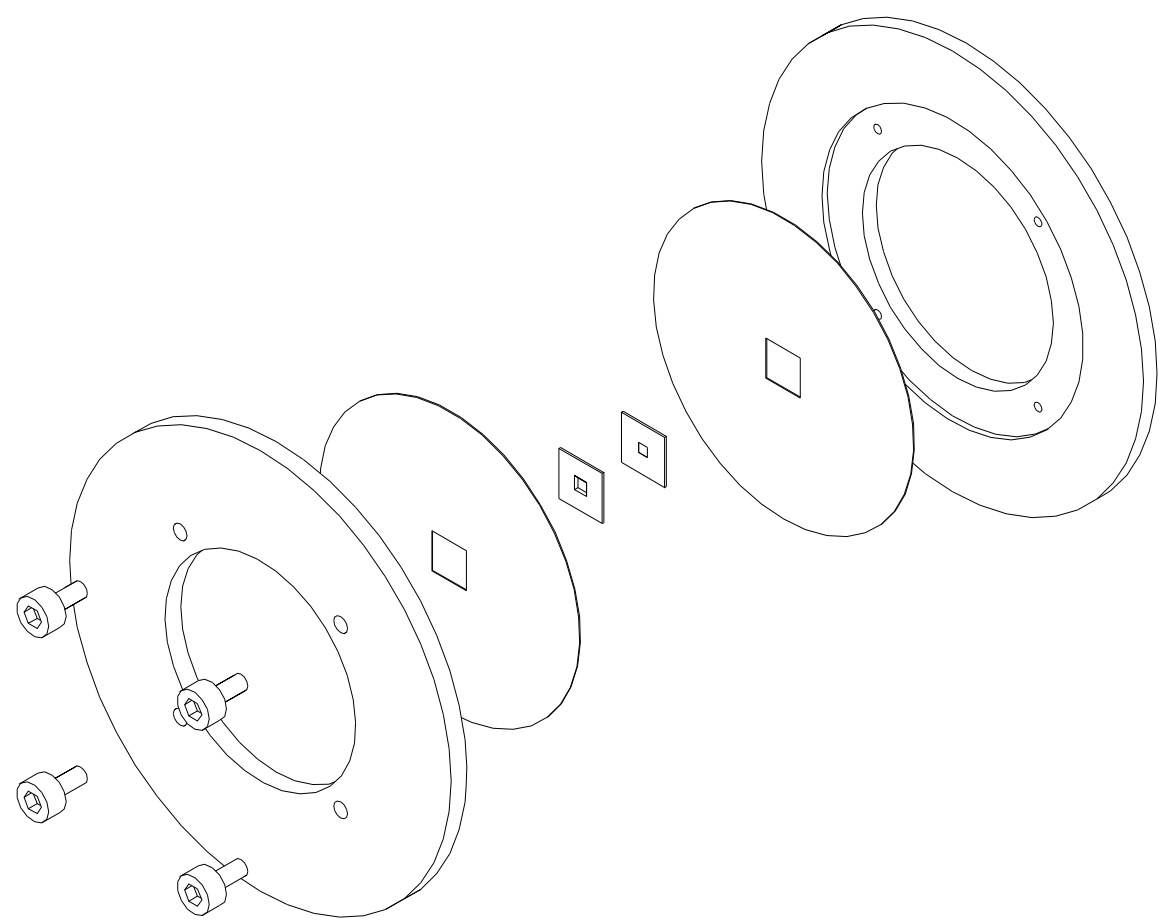

Figure 4.10: Exploded view of the wet specimen chamber as used for the STXM. The specimen is located between two silicon nitride windows, which are glued onto thin metal sheets. The metal sheets are pressed together by the standard TXM wet specimen chamber stainless steel rings.

gap between the OSA and detector vacuum windows with $\mathrm{CO}_{2}$ or other gases for energy calibration and determination of the spectral resolution of the monochromator (see 3.5.2). 


\section{Chapter 5}

\section{The STXM detector}

A detector for scanning transmission X-ray microscopy has to meet several specific requirements. It has to be capable of measuring very high photon rates of up to $10^{9}$ photons/s without reaching saturation. The detector should not add a significant amount of noise to the photon noise. In order to allow fast raster scans of the specimen with short dwell times below $1 \mathrm{~ms}$, the detector readout time has to be short. Finally, the detector has to withstand high radiation doses without degradation of the detection efficiency and without a noise level increase.

On the other hand, for incoherent bright field imaging the detector needs no spatial resolution and even for advanced contrast modes such as phase contrast few detector segments suffice (see Sec. 1.4). Because the radiation is already monochromatized, no spectral resolution is required. The detector may integrate the detected signal during one pixel dwell time, because the time the photons impinge on the detector during the scanning dwell time is of no interest.

In scanning transmission X-ray microscopes, counting detectors such as photomultipliers coupled to quantum converters or gas proportional counters are most commonly used. While counting detectors provide good signal-to-noise ratio at low photon rates, they typically reach saturation at about $10^{6}-10^{7}$ photons $/ \mathrm{s}$ because of the dead time after each registered photon. For the higher photon rates at undulator beamlines at third generation synchrotron sources, integrating detectors have to be employed.

Recently, M. Feser et al. developed an integrating segmented silicon detector specifically for scanning transmission X-ray microscopy used at the Stony Brook STXM at the NSLS $[19,20]$. It consists of eight radiation sensitive segments to get additional contrast modes such as phase or dark field contrast.

For most TXMs, back-illuminated metal-oxide on semiconductor charge coupled device (MOS CCD) detectors are used which integrate the signal during the exposure time. Their operating principle is described in Sec. 5.1. CCDs employed in TXMs have typically $1024 \times 1024$ or $2048 \times 2048$ pixels to record full-field images with high resolution and readout times are of order seconds so they are impractical for use in STXMs and have only been used in particular 

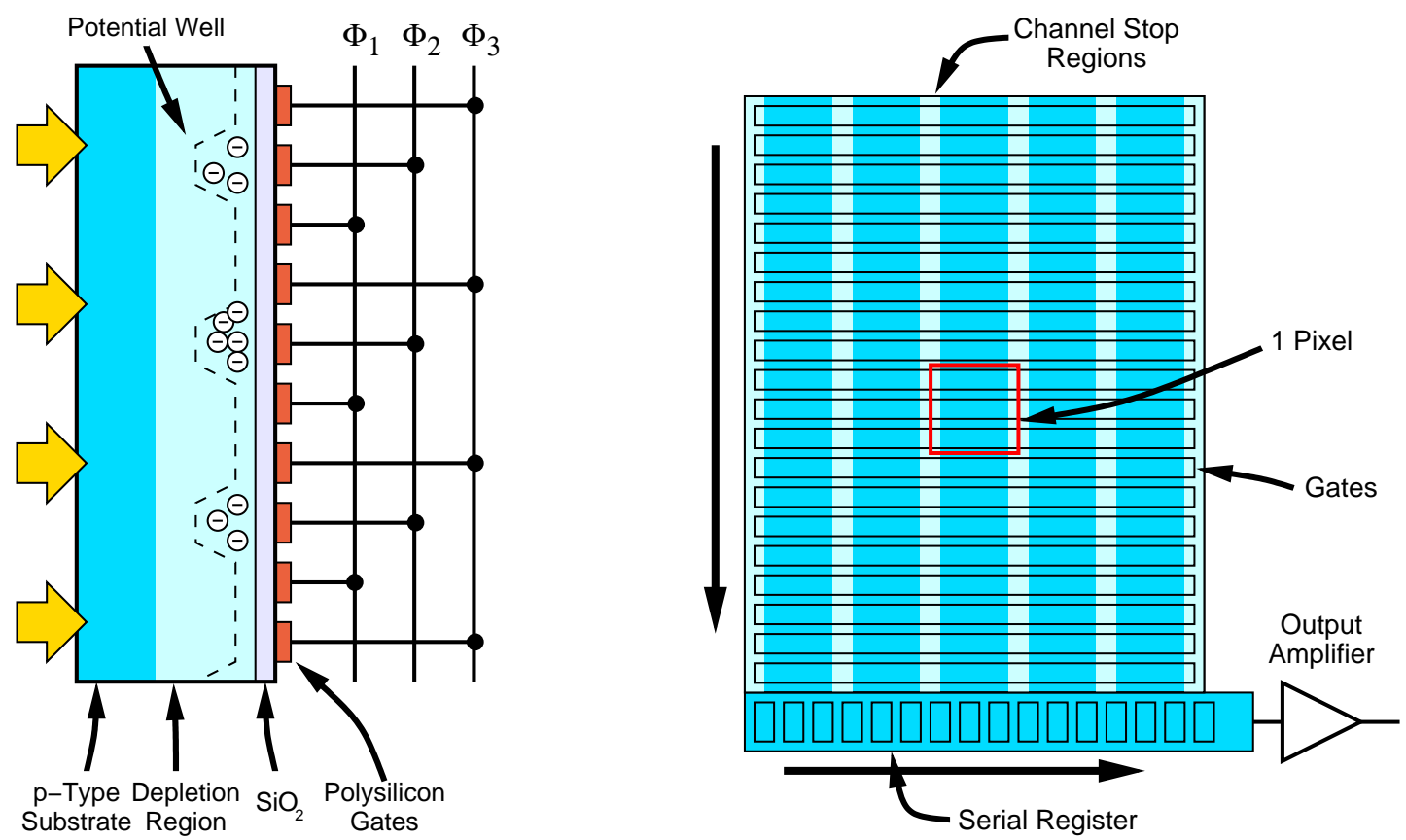

Figure 5.1: Operating principle of the charge coupled device (CCD). Left: schematic cross section of a back-illuminated CCD. Right: readout scheme for a three-phase CCD (top view).

experimental setups $[48,13,14]$.

G. Morrison et al. are using a MOS CCD with only $80 \times 80$ pixels for STXM experiments at the ID21 beamline at the ESRF [7], which is read out in a few milliseconds $[16,49]$. However, MOS CCDs are sensitive to radiation damages, especially at higher photon energies. This limitation is overcome by the pn-CCD detector, which is employed as the detector for the BESSY II STXM (see Sec. 5.2).

\subsection{Operating Principle of the Charge Coupled Device}

CCDs have replaced other imaging devices such as video tubes in most modern general-purpose and scientific cameras. They can be built with high detection efficiency, have good linearity and are free from geometric aberrations. The first CCDs have been developed at Philips [66] and AT\&T [10].

The operating principle of the charge coupled device (CCD) detector is illustrated in Fig. 5.1. In the silicon substrate, the incident photons promote electrons from the valence to the conduction band by the photoelectric effect. The electrons are collected in a potential well during the exposure time. The potential well is created by a MOS (metal oxide on semiconductor) capacitor cell which is formed by the p-type substrate, the $\mathrm{SiO}_{2}$ insulating layer and the gate electrode, which 
is usually made of polysilicon instead of metal in practice. By charging the gate positively, a depletion region of typically $1-5 \mu \mathrm{m}$ thickness is generated below the gate. Electron-hole-pairs generated by the photons in the depleted region are separated by the electric field and the electrons are collected below the positive gate. Electrons generated outside of the depleted region are only collected if they reach the depletion region on their random drift path before recombining.

CCDs are commonly illuminated from the front side through the gate structure. If the CCD is illuminated with X-rays, permanent charges are generated in the $\mathrm{SiO}_{2}$ insulating layer influencing the charge transfer and giving rise to degradation of the imaging properties. Furthermore, the X-rays have to pass through the strongly absorbing gate structure and the insulating layer before reaching the sensitive area of the CCD. Therefore, CCDs employed for X-ray detection are illuminated from the backside. For backside illumination, the silicon substrate has to be thinned to a thickness of $10-20 \mathrm{~nm}$ so that the radiation is not absorbed by the less sensitive, non-depleted silicon.

The number of electrons that can be stored in a potential well, the so-called full well capacity, is proportional to the pixel area. Therefore, CCDs with larger pixels have a larger full well capacity and thus a larger dynamic range.

To read out the generated charge pattern, the electrons are shifted in parallel toward the serial register placed next to the CCD, which may be thought of as a one-dimensional CCD (see Fig. 5.1). Each row transferred to the serial register is then read out serially to the charge sensitive amplifier connected to the end of the serial register. The electrons are laterally confined to the columns by channel stops consisting of highly p-doped regions. The pixel-by-pixel, rowby-row value stream obtained represents the intensity pattern illuminating the CCD. The charges are transferred by applying periodic voltage sequences to the gates, which shift the minimum of the potential wells in the direction of the charge transfer. For scientific CCDs three-phase charge transfer sequences are most commonly used, so they have three gate electrodes per pixel row.

The charge transfer efficiency (CTE) is a measure for the fraction of the charge shifted to the next row. Modern CCDs achieve CTE values better than 0.9999. Because the CTE is raised to the power of the number of shift cycles to get the overall charge transport efficiency, even a CTE of 0.9999 means a $10 \%$ intensity loss for the last pixel of a CCD with 1024 rows.

To reduce the noise level, most scientific CCDs are read out slowly. At a typical readout pixel clock of $1 \mathrm{MHz}$, a $1024 \times 1024$-pixel CCD is read out in about one second. This makes standard CCDs unsuitable for STXMs, where scanning dwell times of order $1 \mathrm{~ms}$ are required.

With the standard MOS capacitor cell, depletion regions of only about $10 \mu \mathrm{m}$ thickness can be generated. At a photon energy of $8 \mathrm{keV}$, the $1 / \mathrm{e}$ absorption length of silicon is $70 \mu \mathrm{m}$, so only $12 \%$ of the radiation are absorbed in the $10 \mu \mathrm{m}$ thick depletion region. To enhance the detection efficiency, CCDs for this energy region are fitted with a so-called quantum converter, usually a phosphor coating 
which converts the high energy X-ray photons into lower energy photons that are detected with high efficiency.

Back-illuminated MOS CCDs have been used with great success in transmission X-ray microscopy in the water window energy region [97]. However, especially at higher photon energies, the photons can pass through the whole silicon substrate and reach the $\mathrm{SiO}_{2}$ insulating layer even in back-illuminated CCDs, so they are susceptible to radiation damage.

The above shortcomings of the slow-scan MOS-CCD are addressed by the development of the pn-CCD.

\subsection{The pn-CCD Detector}

The pn-charge coupled device (pn-CCD) has been developed for space-based Xray astronomy by the Semiconductor Laboratory of the Max-Planck-Institutes for physics and for extraterrestrial physics, München (MPI-HLL) [79]. It has been employed in the European X-Ray Multi-Mirror satellite mission (XMM-Newton) and in the failed ABRIXAS mission. Since it is used to gain spectroscopic information of single photons, the pn-CCD has been developed for fast readout to avoid multiple photons hitting a single pixel during the exposure time. Furthermore, the detector has to be radiation hard to withstand the cosmic radiation. For the use in the satellite telescope focal plane, 12 pn-CCD subunits of $64 \times 200$ pixels each are combined on one silicon wafer to form a $384 \times 400$-pixel detector with a size of $6 \times 6 \mathrm{~cm}^{2}$. The pixel size of $150 \times 150 \mathrm{\mu m}^{2}$ matches the angular resolution of the XMM telescope.

The functional principle of the pn-CCD resembles that of the back-illuminated MOS-CCD, with the main differences being the depletion of the whole substrate thickness, the gate insulation by pn-junctions and the parallel readout of all 64 channels of a row.

The method to obtain full depletion of the pn-CCD substrate, the so-called sideward depletion, has been introduced for silicon drift chambers by Gatti and Rehak [22]. The positive voltage between the highly n-doped anode and the highly p-doped gates and back contact causes a depletion of the whole n-doped substrate (see Fig. 5.2). Therefore, the detector is sensitive to photons in the whole $270 \mu \mathrm{m}$ thick substrate shortly after the about $30 \mathrm{~nm}$ thick $\mathrm{SiO}_{2}$ entrance window and the thin $\mathrm{p}^{+}$implantation, so high efficiencies over an energy range of $0.2-10 \mathrm{keV}$ are obtained (see Fig. 5.12).

The charge generated drifts to the potential minimum in the n-doped epitaxial layer. After exposure, the charge is transferred by periodic signals on the gates to the readout anode analogously to the three-phase MOS CCD. The gates are insulated from the charge-guiding substrate by reverse-biased pn-junctions instead of by a silicon oxide layer. Silicon oxide is only used to insulate the gates among one another and for the entrance window. Therefore, the main source of 


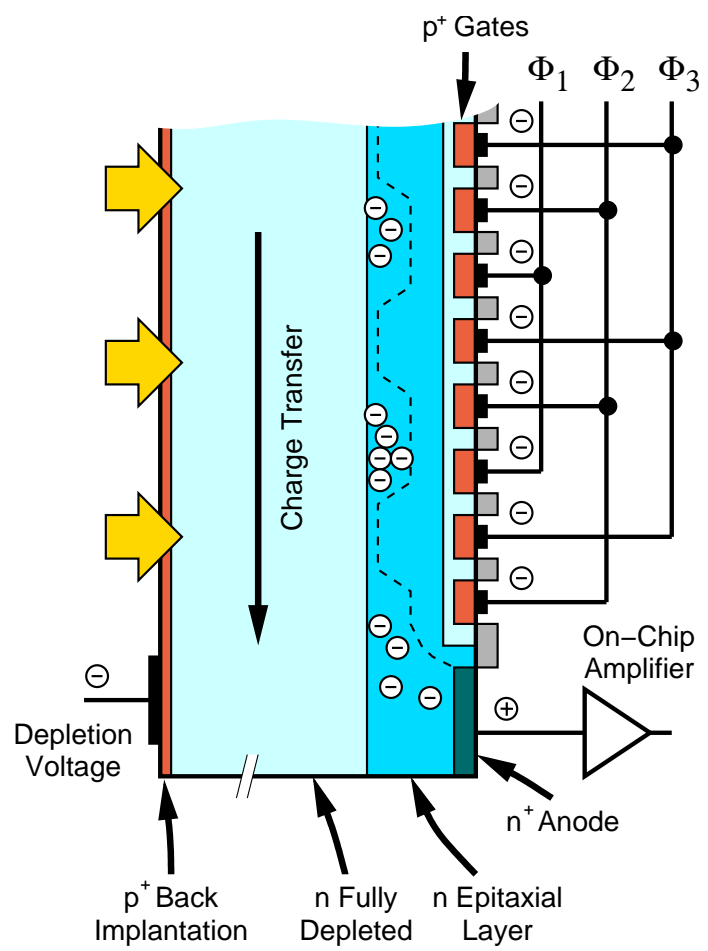

Figure 5.2: Schematic cross section of the pn-CCD. The fully depleted $n$ silicon detector volume is $270 \mu \mathrm{m}$ thick, the $\mathrm{n}$ epitaxial layer is $12 \mu \mathrm{m}$ thick.

radiation damages is eliminated.

Instead of being transferred to a serial register as in the standard MOS CCD, the charge reaching the 64 anodes is read out in parallel (see Fig. 5.3). The charge of one pixel row shifted to the anode is pre-amplified by the first FETs on the pn-CCD chip. After the readout of each row, the remaining charge on the anode can be reset by the Reset FET. The output signal is amplified and multiplexed on the CAMEX chip. The CAMEX amplification factor can be lowered by a factor of 20 to be able to handle larger photon numbers. The timing for the CAMEX digital signals is generated by the TIMEX chip.

\subsubsection{The pn-CCD Detector Used for the BESSY II STXM}

In the BESSY II STXM, a single pn-CCD subunit with $64 \times 200$ pixels is employed to detect the X-rays transmitted through the object. During the charge transfer, one row is read out in $28.5 \mu \mathrm{s}$, so a whole frame with 200 rows is read out in $t_{\mathrm{r}}=5.7 \mathrm{~ms}$. The exposure time $t_{\mathrm{e}}$ set with the detector electronics gives the period of the frame readout. Hence, for an exposure time of $t_{\mathrm{e}}=6 \mathrm{~ms}$, which has been used as the standard exposure time for the first experiments, the generated charge accumulates for $t_{\mathrm{e}}-t_{\mathrm{r}}=0.3 \mathrm{~ms}$ and is then read out in $5.7 \mathrm{~ms}$. The pn-CCD is sensitive to X-radiation during the charge transfer, so the diffraction 


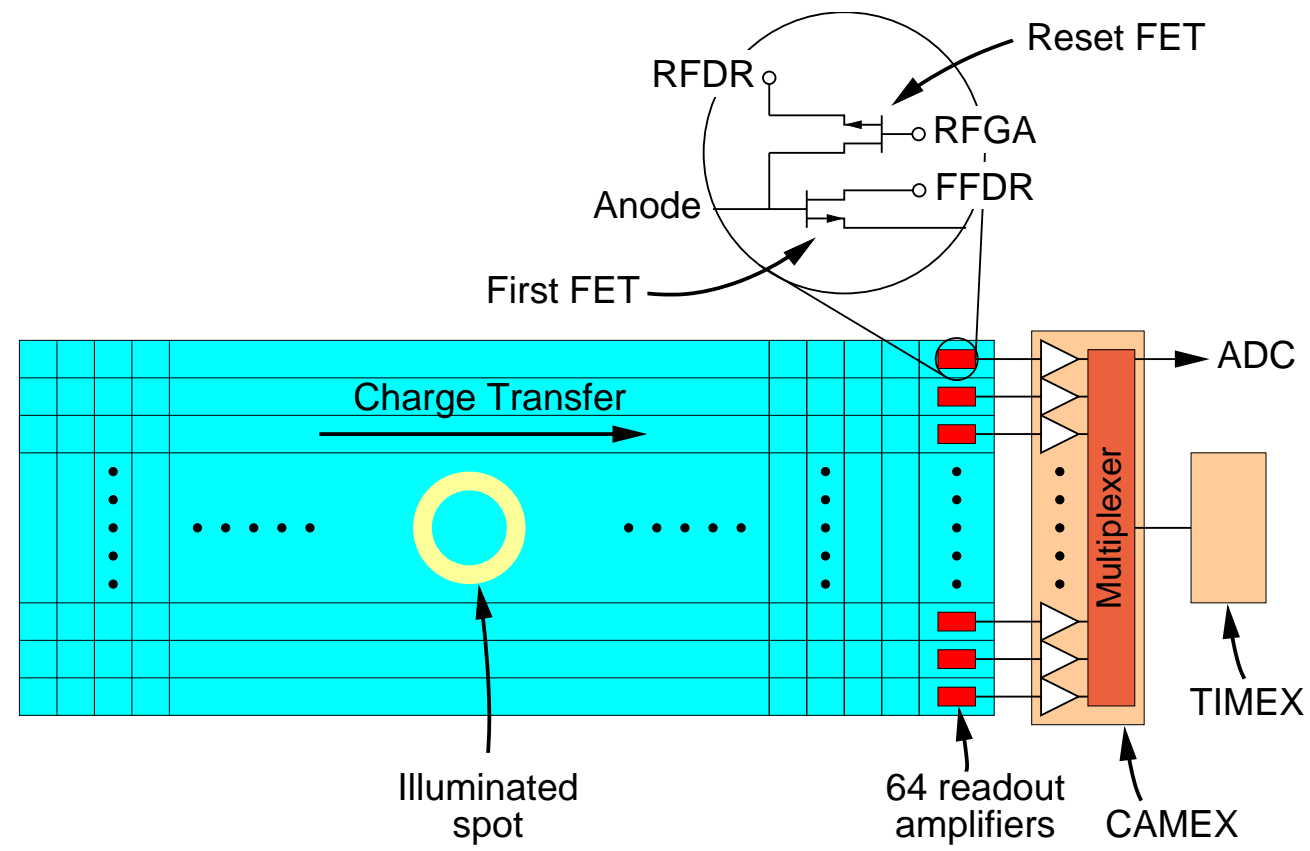

Figure 5.3: Schematic drawing of a single $64 \times 200$-pixel pn-CCD subunit with the CAMEX amplifier/multiplexer and TIMEX timing generator as employed in the STXM. The schematic of one on-chip readout amplifier is shown magnified with the first FET and the Reset FET.

pattern leaves a stripe parallel to the charge transfer direction in addition to the two-dimensional diffraction pattern. Fig. 5.4 shows the calculated charge pattern read out with the pn-CCD at three different exposure times.

If the exposure time is equal to the readout time, the detector is read out continuously. However, with continuous readout the two-dimensional position sensitivity of the pn-CCD is lost and the diffraction pattern is resolved only in the direction perpendicular to the charge transfer. At a photon energy of $283 \mathrm{eV}$ and at a distance of $20 \mathrm{~mm}$ of the detector from the specimen, the spot illuminated by the hollow cone from the zone plate has a diameter of 12 pixels (see Fig. 5.4). Therefore, the pixel dwell time for the raster scan with continuous detector readout must be larger than $12 \times 28.5 \mu \mathrm{s}=0.34 \mathrm{~ms}$. Because the minimum pixel dwell time is proportional to the diameter of the diffraction pattern, the detector has to be placed as close as possible to the specimen to obtain short readout times. The STXM pn-CCD is normally used in continuous readout mode to allow short scan dwell times; longer exposure times are used for contrast modes requiring two-dimensional diffraction images such as differential phase contrast (see Sec. 1.4.1).

The maximum number of photons is not limited by the CCD full well capacity, which is very high because of the large pixel area, but by the amplifiers on the CAMEX chip. The CAMEX amplification factor can be set to two different values differing by a factor of 20. For operation in the STXM, the lower amplification 

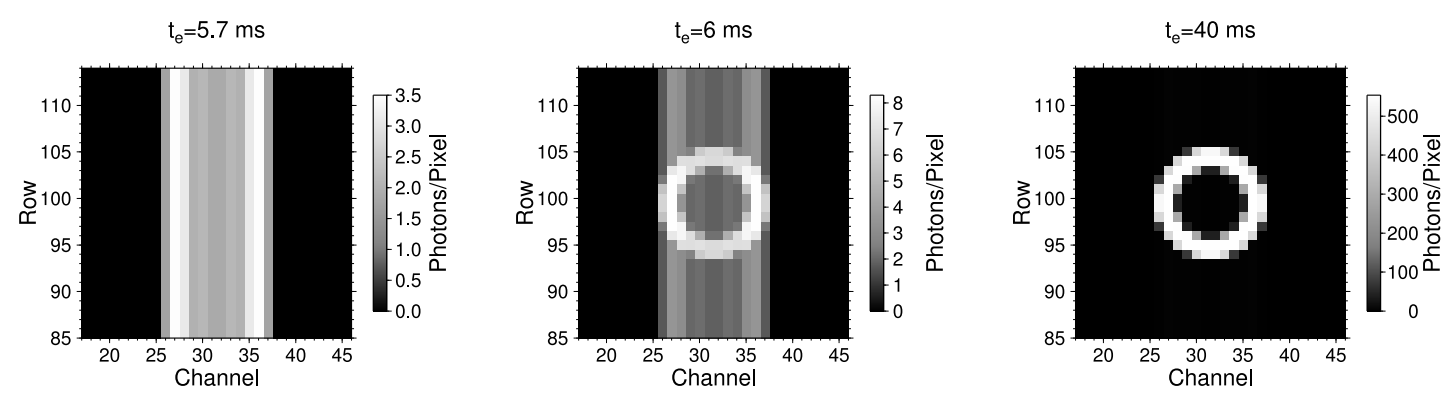

Figure 5.4: Calculated number of photons per pn-CCD pixel for exposure times of $t_{\mathrm{e}}=5.7 \mathrm{~ms}$ (continuous readout), $t_{\mathrm{e}}=6 \mathrm{~ms}$ and $t_{\mathrm{e}}=40 \mathrm{~ms}$ with an incident photon rate of $10^{6} / \mathrm{s}$. Note the different intensity scales. Only the central $29 \times 29$ pixels of the $64 \times 200$-pixel pn-CCD are shown. The illumination is calculated for a photon energy of $283 \mathrm{eV}$, a distance between specimen and pn-CCD of $20 \mathrm{~mm}$ and a zone plate with $\mathrm{d} r_{N}=50 \mathrm{~nm}, r_{N}=100 \mu \mathrm{m}$ and $r_{\mathrm{CS}}=65 \mu \mathrm{m}$.

is used to allow larger photon rates. The measured maximum photon rates are given in Sec. 5.4.2.

To reduce the electronic noise level, the detector is cooled with liquid nitrogen to about $-120^{\circ} \mathrm{C}$. Therefore, it must be kept in vacuum to avoid condensation of the air humidity on the detector surface. When the liquid nitrogen tank is filled, it acts as a cooling trap and lowers the pressure from $2 \times 10^{-7} \mathrm{mbar}$ to about $3 \times 10^{-8}$ mbar. Fig. 5.5 shows the pn-CCD in the STXM detector vacuum vessel with the liquid nitrogen tank.

The pn-CCD (a), the CAMEX and the TIMEX chips are mounted on a 40-pin ceramic dual-in-line carrier pressed against a copper heat sink (c). A $3 \mathrm{~mm}$ thick copper sheet metal is mounted on top of the pn-CCD ceramic and the heat sink to cool the detector from the front side.

The radiation from the sample passes through the pinhole with the detector vacuum window (b, see Sec. B.2). The copper heat sink is mounted on the in-vacuum printed circuit board (PCB) (d). Six copper strands (not shown) thermally couple the heat sink to a copper block (e) mounted below the stainless steel liquid nitrogen tank (f). The temperature of the copper block is measured with a Pt100 sensor. The in-vacuum PCB is mounted on an X-Y stage (g) which is used to position the desired area of the pn-CCD in the beam. The X-Y stage with the detector can be moved in $\mathrm{Z}$ direction from the air side with a linear feedthrough (h). The minimum distance of the pn-CCD surface from the sample is $13 \mathrm{~mm}$. An electric feedthrough (i) for the pn-CCD voltages is located on the opposite side of the detector.

The detector vacuum vessel is mounted on an X-Y stage adjustable with micrometer precision, so the detector pinhole can be adjusted to the optical axis. The X-Y stage is mounted on a linear guidance with $400 \mathrm{~mm}$ travel to set the $\mathrm{Z}$ distance of the detector pinhole from the specimen and to retract the detector 


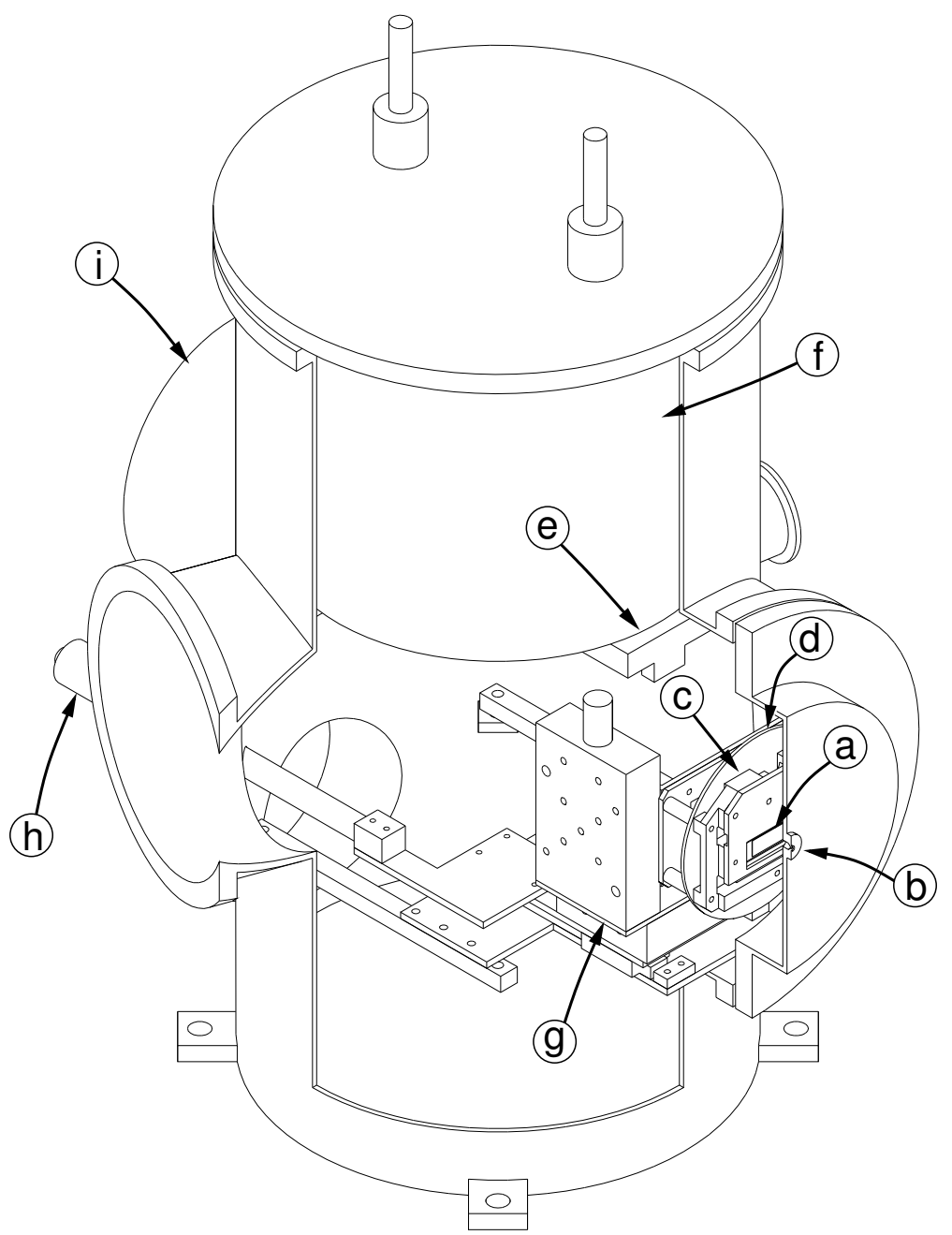

Figure 5.5: Cut away drawing of the pn-CCD detector vacuum vessel. See text.

vessel for previewing the specimen with the visible light microscope (see Fig. 2.3). The linear guidance is driven by a Faulhaber DC motor (3863 A $024 \mathrm{C}$ ) with a MCDC 2805 controller. Close to the specimen, the detector vessel $\mathrm{Z}$ position is measured with a Heidenhain position encoder to control the distance of the air gap between the zone plate and detector pinholes with micrometer-accuracy.

\subsection{The Front End Electronics for the pn-CCD}

Fig. 5.6 shows an schematic overview of the detector electronics. On the air side of the vacuum feedthrough, a printed circuit board is mounted in a aluminum casing (not shown), where the cables leading to the front end electronics are plugged in.

The front end electronics have been developed by H. Gorke at the Forschungs- 


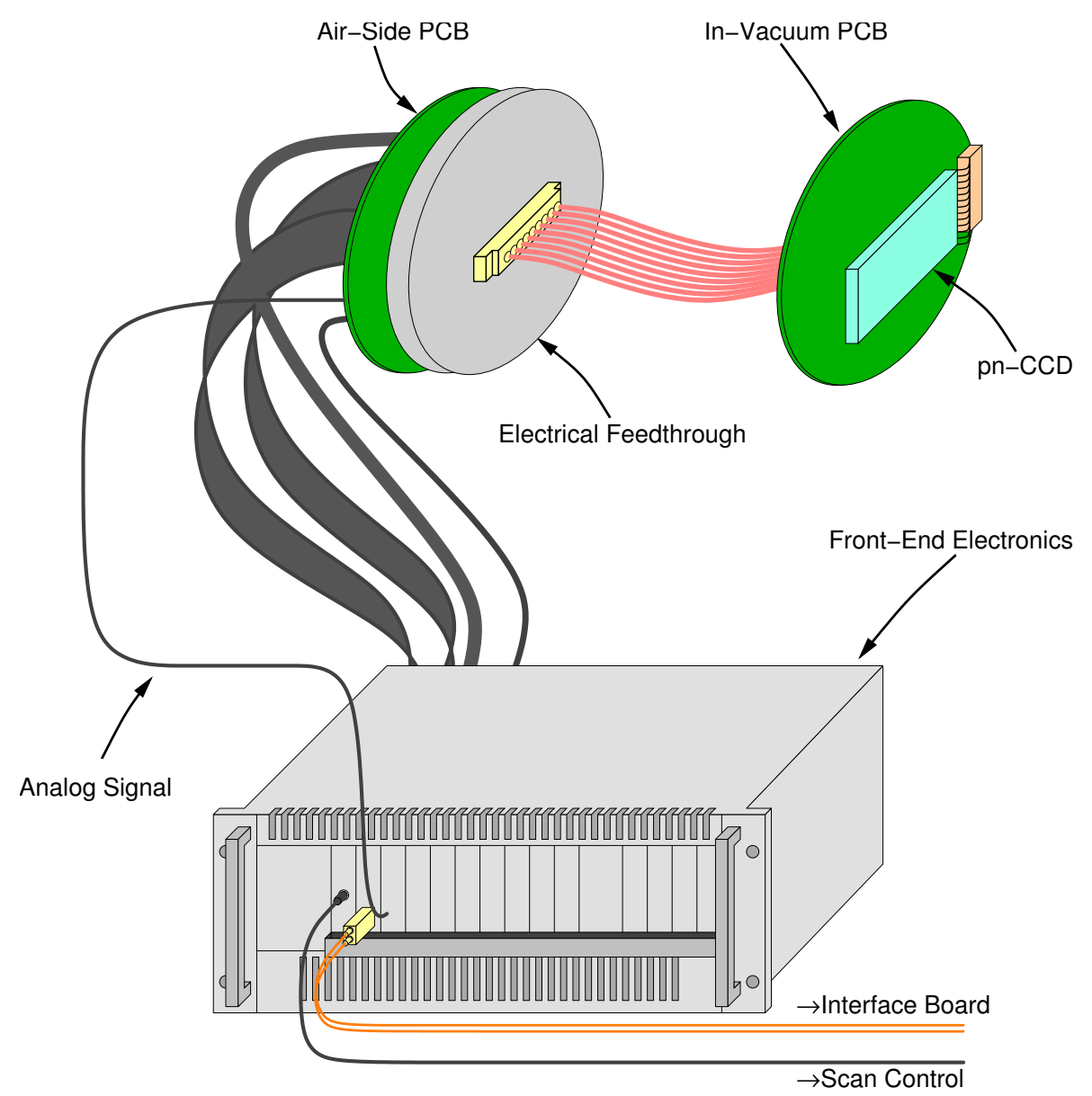

Figure 5.6: Schematic overview of the pn-CCD electronic components with the invacuum and air-side printed circuit boards and the front-end electronics.

zentrum Jülich. The electronics box consists of several interchangeable plug-in modules connected by a Siemens SMP bus:

- The sequencer to generate the various signals for the CCD charge transfer and readout

- A 12-bit analog to digital converter (ADC) to digitize the measured signal

- Adjustable low-noise DC power supplies

- Voltage, current and temperature measurement

- An optical fiber interface to the control computer

- A micro-controller for the control of the power-on and power-off procedure The modules can be exchanged easily for repair or upgrade. All parameters are set from the control computer making the system less susceptible to handling 


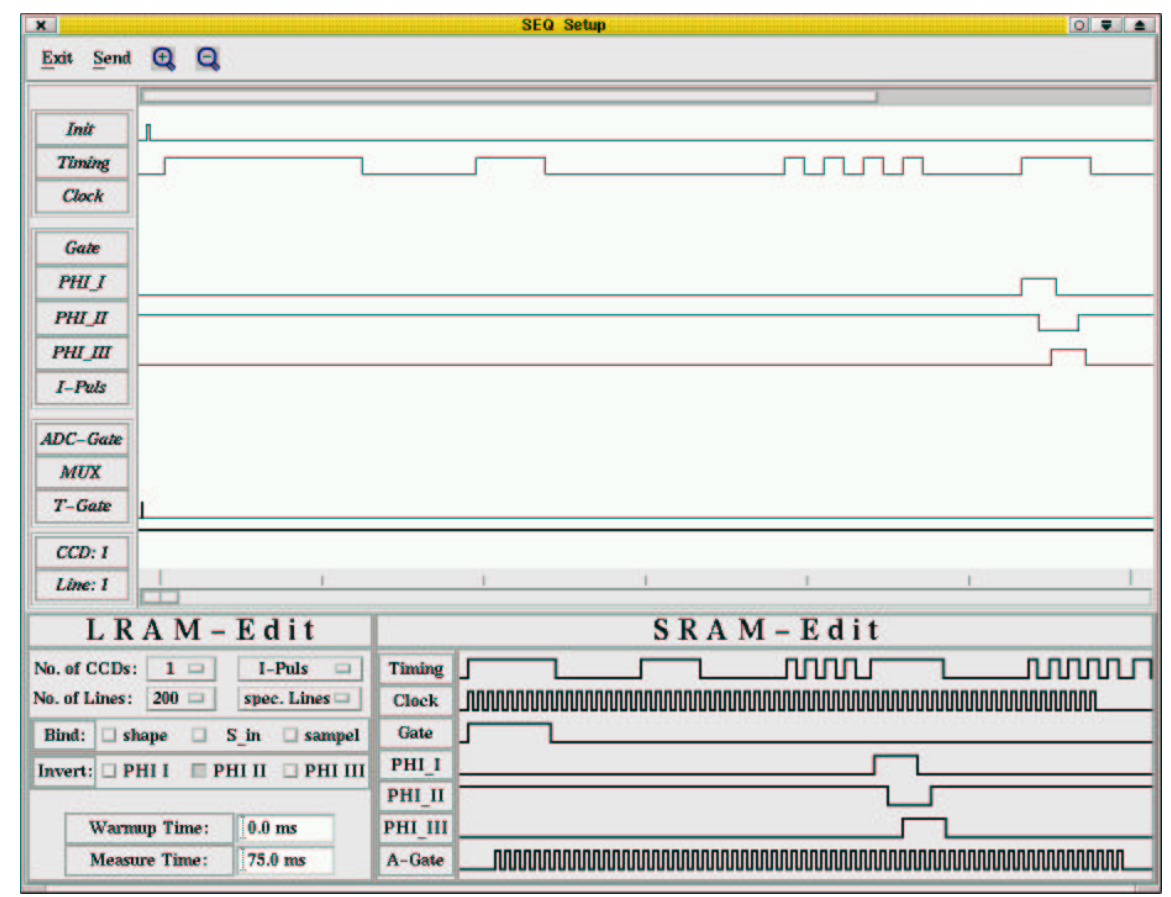

Figure 5.7: Screen shot of the SEQ_setup program used to create and modify the sequencer signals. The gate signals of one CCD shift cycle are visible (PHI_I to PHI_III).

errors. During the power-on and power-off procedures, the CAMEX and pnCCD voltages are set in a defined sequence to avoid damage to the pn-CCD. The parameters for different operating modes are kept in parameter files on the control computer, so the microscope user only has to start the CAMEX and pn-CCD startup routine in the control program. The micro-controller permits shutting down the CCD autonomously following a procedure stored in flash EPROM for emergency cases, e.g. during power failures. To keep the front-end electronics running during the shut-down procedure in case of a power failure, it is connected to an uninterruptible power supply.

The sequencer can be programmed to generate arbitrary signals. Fig. 5.7 shows a screen shot of the sequencer setup program. A possible modification of the standard readout sequence would be the division of the 200-row frame into smaller areas to obtain shorter scan pixel dwell times while still registering twodimensional diffraction images at each scan pixel. For example, one could expose 20 rows for $0.5 \mathrm{~ms}$ without charge transfer and then shift the charge 20 rows farther in $20 \times 28.5 \mu \mathrm{s}=0.57 \mathrm{~ms}$. This means a minimum scan dwell time of $1.07 \mathrm{~ms}$ compared to $6.2 \mathrm{~ms}$ for full-frame readout with $0.5 \mathrm{~ms}$ charge accumulation time.

When the data acquisition is started, the sequencer sends a TTL pulse generating an interrupt at the scan control computer which starts the scan sequence at a defined time interval after the first pn-CCD frame is read out.

The analog signal from the detector is converted to a 12-bit digital value. An 
adjustable offset voltage can be subtracted from the signal prior to the conversion to be able to take advantage of the whole range of the analog to digital converter. The optical fiber interface module sends the measured values to the interface board in the control computer. The interface board writes the data continuously via direct memory access (DMA) to a ring buffer in the computer memory. After an adjustable number of frames, an interrupt is generated signalling the readout program running on the computer to process the incoming data. For each pixel, the row and channel number, an under- and overflow bit and the 12-bit pixel intensity are encoded into four bytes in the front-end electronics interface module.

\subsubsection{Processing of the pn-CCD data}

When reading out continuously, the detector generates a data stream of $9 \mathrm{Mbyte} / \mathrm{s}$. Before storing it on the hard disk, this data is verified, corrected and reduced.

First, the row and channel numbers encoded in the raw pixel data are used to check whether the stream is continuous and no pixels or rows are lost or sent multiple times. The overflow and underflow bits are evaluated and the 12-bit integer intensity is converted to a floating point representation for the following processing steps.

For each exposure time, each pn-CCD pixel has a specific zero offset determined by averaging the pixel values of a number of pn-CCD frames without illumination. The zero offsets are subtracted from the raw data. The resulting intensities can be corrected for the specific amplification of each CAMEX channel (see Fig. 5.8).

Because of low-frequency pickup, the intensity of each row read out is shifted by a certain amount, the so-called common noise. Because only the center pixels of a row are illuminated by the hollow cone from the zone plate, the outer pixels may be used to calculate the common noise level, which is then subtracted from the row pixel values. To discard outliers caused e.g. by cosmic particles, the outer pixel values are sorted and the mean of the center six values is taken for the offset correction.

In the next step, the relevant area of the pn-CCD frame is cut out. For long exposure times, one can cut out only the channels and rows around the diffraction pattern. For shorter exposure times, when a large part of the photons reaches the detector during the readout charge transfer, all rows have to be used to avoid a loss of detected photons.

The resulting data stream can either be saved as a whole for later processing or it can be reduced to a few intensity values per frame with selectable detector response functions during the image acquisition. Interesting detector response functions are among others the intensity sum of the whole detector for bright field imaging, the first moments in horizontal and vertical direction for differential phase contrast, and the sum of the pixels outside of the bright field hollow cone for dark field imaging (see Sec. 1.4.1). 


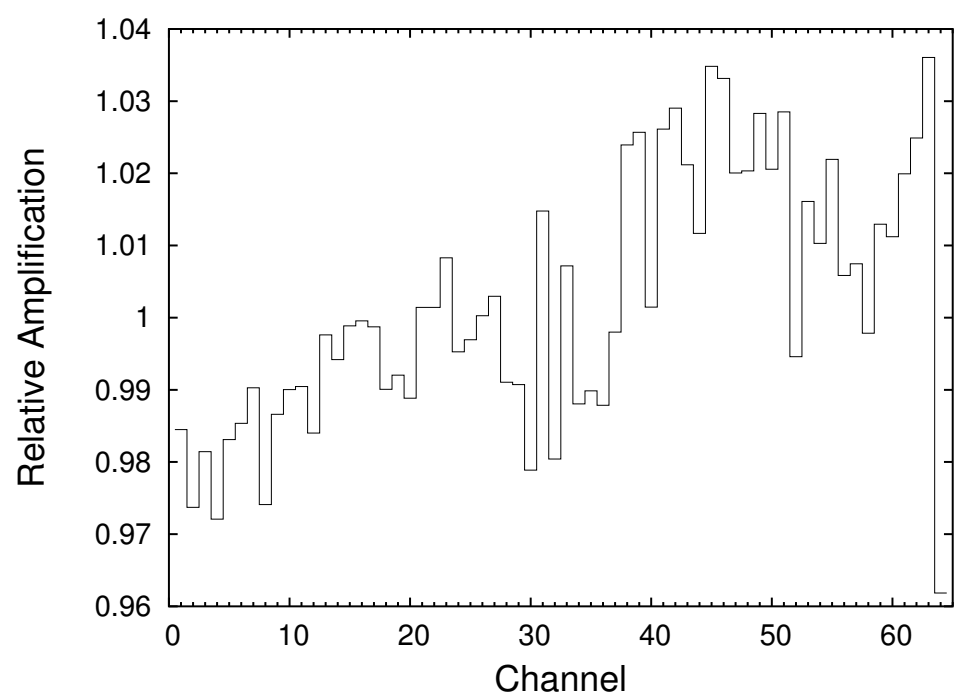

Figure 5.8: Relative amplification of the 64 channels of the STXM pn-CCD [30].

\subsection{Characterization of the PN-CCD Detector}

Two different X-ray sources are used for the characterization of the pn-CCD: Monoenergetic photons generated by a $\mathrm{Fe}^{55}$ source are used for the measurement of the noise, the charge transfer efficiency and the amplification of the detector. The quantum detection efficiency and the high rate stability are examined using synchrotron radiation.

\subsubsection{Detector Calibration with a $\mathrm{Fe}^{55}$ source}

The detector is calibrated using the $\mathrm{Mn} \mathrm{K}-\alpha$ radiation of a $\mathrm{Fe}^{55}$ source, which has a photon energy of $5899 \mathrm{eV}$. Because of the low photon rate, spectra of single pixels can be acquired. In high gain mode, a photon with an energy of $5899 \mathrm{eV}$ generates a signal of 650 analog to digital converter (ADC) counts. In the most commonly used low gain mode, where the gain is reduced by a factor of 20 to allow higher count rates, this corresponds to a conversion factor of 0.00551 counts $/($ photon $\times \mathrm{eV})$. With this conversion factor and the quantum detection efficiency (see Sec. 5.4.2) the incident photon rate can be calculated from the intensity measured in ADC counts.

By comparing the spectra acquired for the 64 channels, the relative amplification can be calculated (see Fig. 5.8). The relative amplification ranges from 0.96 to 1.04 . The pixel intensities can be corrected for the amplification factors during the data acquisition (see Sec. 5.3.1).

Fig. 5.9 shows the charge transfer efficiency (CTE, see Sec. 5.1) of the 64 channels of the STXM pn-CCD calculated from the $\mathrm{Fe}^{55}$ pulse height decay along the 200 pixels of each channel. The mean charge transfer efficiency is 0.999697 . If 


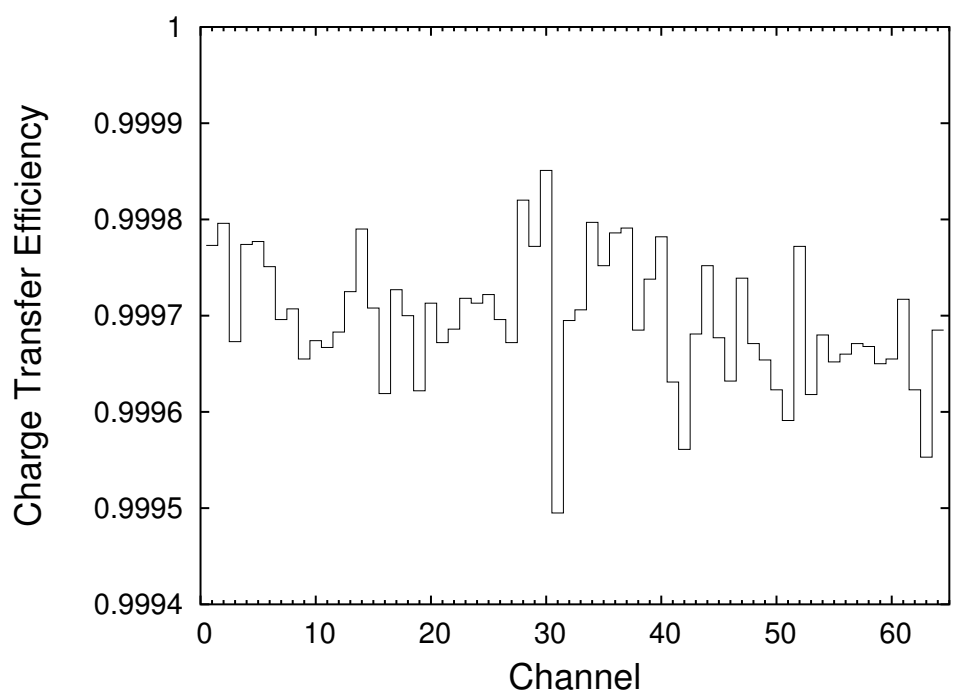

Figure 5.9: Charge transfer efficiency of the 64 channels of the STXM pn-CCD [30]. The mean charge transfer efficiency is 0.999697 .

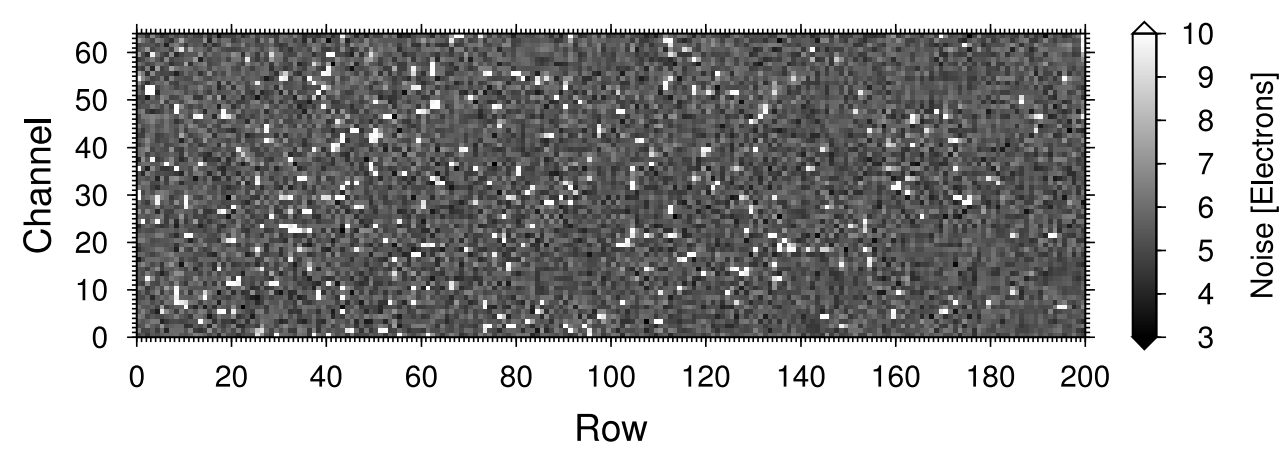

Figure 5.10: Measured noise level of the $64 \times 200$ pixels of the STXM pn-CCD detector [30]. The noise is calculated from the widening of the Mn K- $\alpha$ line recorded at each pixel. The mean pixel noise is 7.0 electrons

an area of 20 rows is illuminated, this means a signal loss of $1-0.999697^{20}=0.6 \%$ of the last row read out compared to the first row. If two-dimensional diffraction patterns are recorded (as opposed to continuous readout), the recorded data can be corrected using the measured CTE.

The noise of the pn-CCD pixels is calculated from the widening of the Mn K- $\alpha$ line in the recorded spectra (see Fig. 5.10). A histogram of the noise levels is shown in Fig. 5.11. The mean noise is 7.0 electrons. The vast majority (96\%) of the pixels have a noise level below 6.5 electrons; the noise level of all but six pixels is lower than 84 electrons.

To liberate one electron-hole pair, $3.65 \mathrm{eV}$ of X-ray photon energy are required. Therefore, a photon with an energy of $283 \mathrm{eV}$ generates 76 electrons, so the average pixel noise lies about one order of magnitude below the signal generated by a 


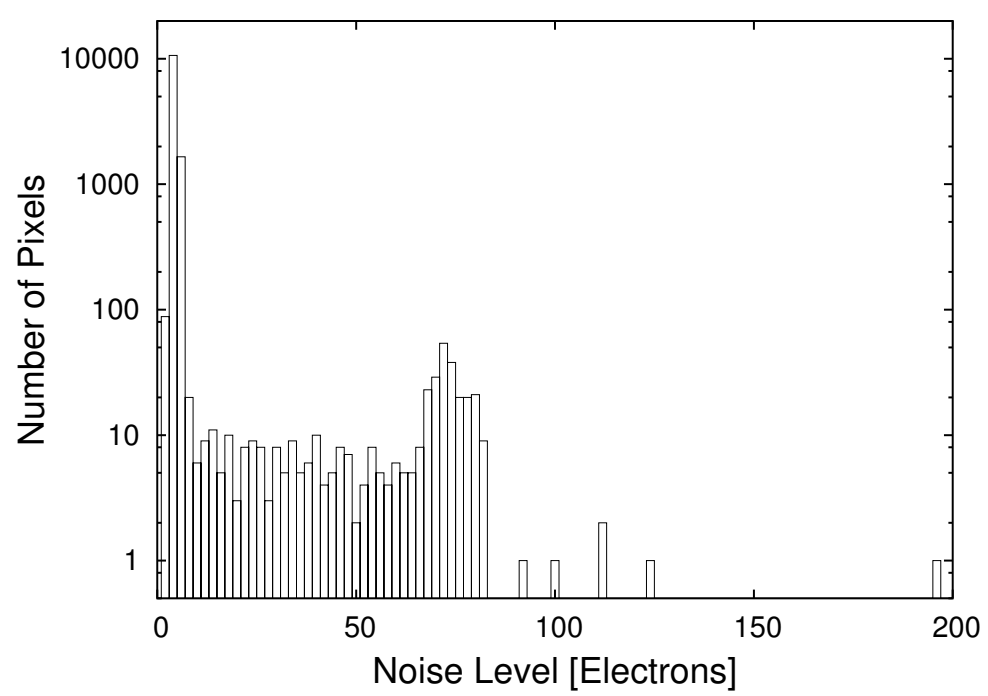

Figure 5.11: Histogram of the STXM pn-CCD pixel noise levels (see Fig. 5.10). Note the logarithmic scale of the ordinate.

photon. One could illuminate an area with low noise levels to further reduce the noise added by the detector, e.g. the region around channel 15 and row 90 (see Fig. 5.10).

The noise of the pixel intensity when acquiring STXM images or spectra is influenced not only by the photon statistics and the detector noise, but also by the stability of the illumination. Therefore, to estimate the signal-to-noise ratio obtainable in images, the fluctuation of the measured intensity has to be measured in the STXM setup with synchrotron radiation.

\subsubsection{Tests with Synchrotron Radiation}

In practice, only a fraction of the photons impinging on the detector are registered because of the entrance window absorption. Furthermore, a part of the charge generated in the detector volume is lost during the transfer to the readout anode. The quantum detection efficiency (QDE) is a measure for the loss of the detected signal because of these two effects; it is defined as the ratio of the number of electrons detected in the real detector to the number of electrons detected with an ideal detector without absorption and with perfect charge transfer. Therefore, together with the conversion factor from photons to ADC counts (see Sec. 5.4.1), the QDE can be used to convert the measured intensity into an absolute photon number. Another efficiency measure frequently used to describe CCD detectors is the detective quantum efficiency (DQE), which gives the degradation of the signal-to-noise ratio of the measured signal due to the detector [96].

The quantum detection efficiency has been measured by Hartmann et al. [29] for a pn-CCD with entrance window properties similar to those of the STXM 


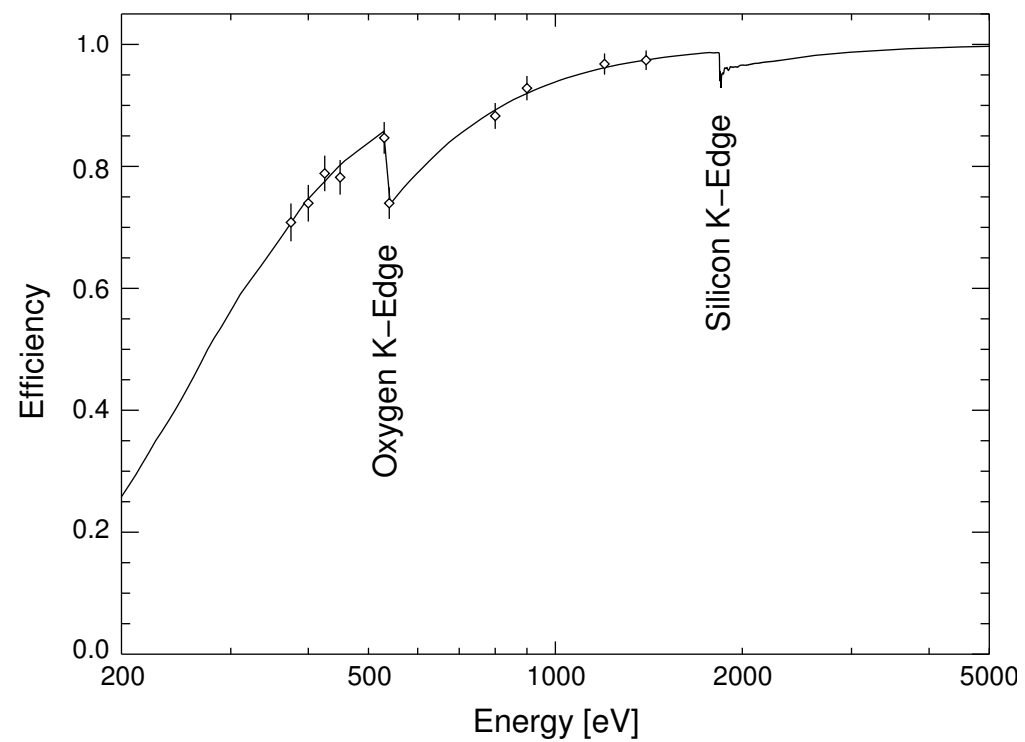

Figure 5.12: Measured quantum detection efficiency of a pn-CCD with similar properties to the pn-CCD employed in the STXM [29]. Points: measured quantum detection efficiency. Line: calculated efficiency fitted to measured points.

pn-CCD (see Fig. 5.12). The QDE is affected mainly by the absorption of the $\mathrm{SiO}_{2}$ entrance window, so silicon and oxygen $\mathrm{K}$ absorption edges are visible. A function taking into account the absorption and charge transfer is fitted to the measured points. It gives an extrapolated quantum detection efficiency at the carbon absorption edge $(283 \mathrm{eV})$ of $52 \%$.

The effectiveness of the pn-CCD anode reset mechanism via the Reset FET has been tested with the STXM setup at a photon energy of $280 \mathrm{eV}$. Fig. 5.13 shows the measured intensity as a function of time for several three different of the Reset FET gate pulses (see Fig. 5.3). Without resetting the anode (RFGA_HI $=-7 \mathrm{~V}$ ), the measured intensity decays within $200 \mathrm{~ms}$ to about half the initial value after the beamline shutter is opened and overshoots into the negative range when the shutter is closed. This effect is attributable to the charge accumulated at the pn-CCD readout anodes. The anodes are read out differentially with the charge before the row transfer to the anode taken as the reference value. If the charge cannot flow off the anode between subsequent readouts, the reference value is larger than zero, and the intensity measured decreases. If the incident photon intensity drops to zero, a positive reference value is subtracted from zero, so the measured intensity becomes negative.

If the Reset FET is triggered, the decay and the negative overshoot can be reduced significantly, but a long positive trail of approximately $10 \%$ of the intensity with open shutter appears. This behavior can be explained by an imperfect charge transport of the pn-CCD. It can possibly be remedied by changing the voltages relevant for the pn-CCD charge transport. After the initial decay, the 


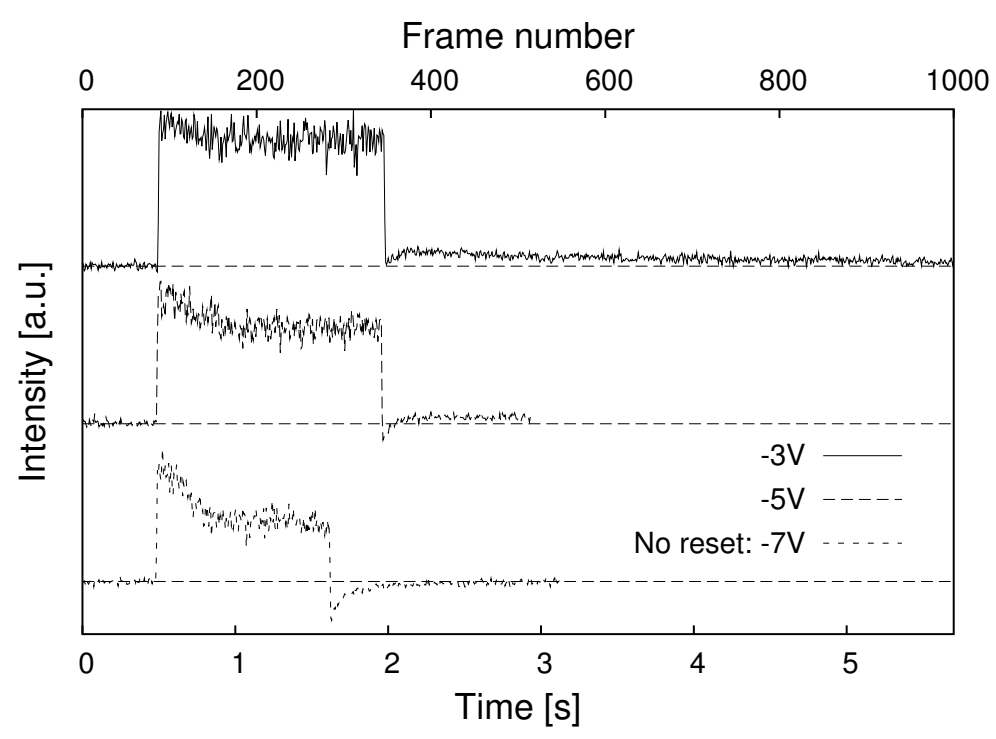

Figure 5.13: Test of the pn-CCD Reset FET mechanism. The detector is illuminated with $280 \mathrm{eV}$ X-rays and read out continuously. The beamline shutter is opened and closed once. The Reset FET gate voltage oscillates between RFGA_LOW voltage, which is set to $-7 \mathrm{~V}$, and RFGA_HI. Shown are the intensities in ADC-counts per frame without active anode reset (RFGA_HI $=-7 \mathrm{~V}$ ) and for anode reset with two different RFGA_HI voltages.

intensity with a voltage of RFGA_HI $=-3 \mathrm{~V}$ is more than twice as high than the intensity without anode reset.

Fig. 5.14 shows a measurement of the pn-CCD linearity for several RFGA_HI voltages. The X-ray intensity is varied by changing the air gap between the OSA and the detector vacuum window. With active anode reset, the progression of the measurements deviates clearly from the theoretical curve at higher air gaps (corresponding to lower intensities), so the detector does not behave linearly. This non-linearity could be explained by an incorrect setting of the analog-to-digital conversion time and could be avoided with a modified sequencer configuration.

The maximum photon rate up to which the detector is linear can be calculated from the saturation visible in Fig. 5.14. With an exposure time of $40 \mathrm{~ms}$, saturation is reached at about $2 \times 10^{-5}$ counts/frame. At an exposure time of $t_{\mathrm{e}}=40 \mathrm{~ms}$, one pixel of the annular diffraction pattern (see Fig. 5.4) is illuminated with $1 / 72$ of the intensity of the whole frame. Therefore, saturation occurs at 2800 counts/pixel or 1400 photons/pixel at $430 \mathrm{eV}$, assuming that the saturation affects only these brightest pixels. With continuous readout, where the maximum intensity per pixel is only $1 / 1646$ of the frame intensity, this corresponds to a saturation photon rate of $4 \times 10^{8}$ photons $/ \mathrm{s}$ at $430 \mathrm{eV}$ or $6 \times 10^{8}$ photons $/ \mathrm{s}$ at $283 \mathrm{eV}$. 

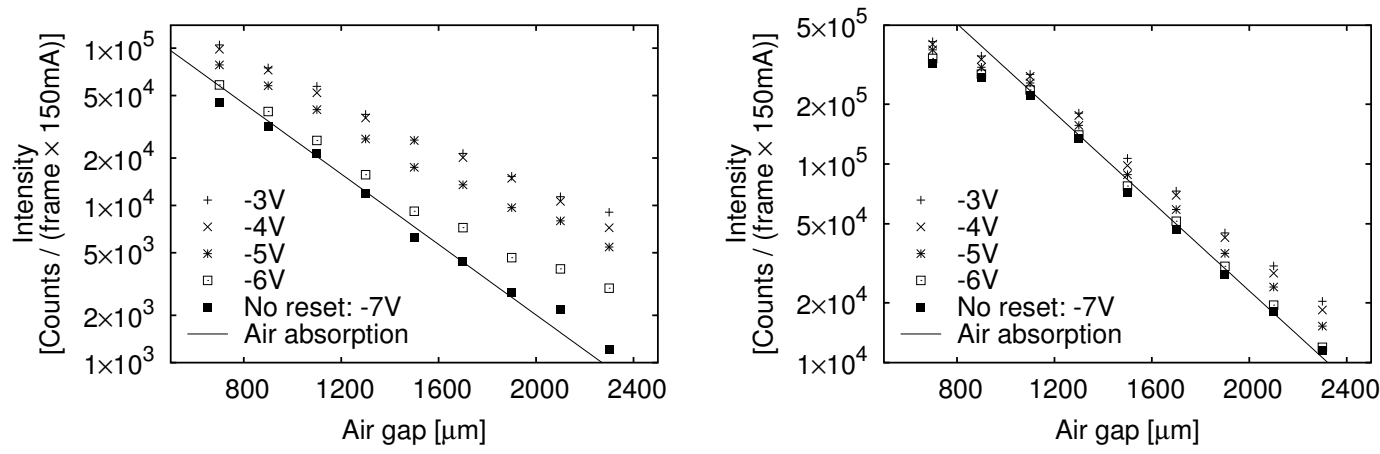

Figure 5.14: Test of the pn-CCD linearity with continuous readout (left) and with an exposure time of $t_{\mathrm{e}}=40 \mathrm{~ms}$ (right) for several RFGA_HI voltages. Shown are the intensities in ADC-counts per frame normalized to a beam current of $150 \mathrm{~mA}$ as a function of air gap. For comparison, a straight line with a slope calculated from the tabulated air absorption [31] is shown. The detector is illuminated with X-rays of an energy of $430 \mathrm{eV}$. 



\section{Chapter 6}

\section{First Measurements with the STXM}

In this chapter, STXM test results on illumination uniformity, absence of distortions, and spectral resolution are shown, before images in elemental contrast and differential phase contrast are presented. Gold grids and diatoms are used as regular structures to test whether the motor and the piezo scan introduce distortions in the image. To measure the achievable spatial resolution using power spectra, gold spheres with a diameter of $250 \mathrm{~nm}$ are imaged, which are also used to check whether the zone plate shows astigmatism. Chernozem dry samples are imaged in elemental contrast and differential phase contrast.

Fig. 6.1 shows a coarse motor scan of a test object consisting of a gold honeycomb grid with a dry chernozem soil sample on a silicon membrane. No distortions of the grid are visible indicating that the pn-CCD readout is well synchronized with the scanning motion and the object moves steadily during the scan. At the right edges of strongly absorbing specimen parts, bright seams are visible. This is caused by charge accumulation at the pn-CCD readout anode (see Sec. 5.4.2), which was not reset with the Reset FET during the measurements presented in this chapter.

To verify that the monochromator illuminates the zone plate homogeneously over the whole scan range, a piezo scan image is acquired with no sample present (see Fig. 6.2). Eqs. (4.1) to (4.3) give a maximum image diameter of $45 \mu \mathrm{m}$ for an OSA diameter of $85 \mu \mathrm{m}$. Apart from noise, the intensity distribution is perfectly uniform in this range.

Fig. 6.3 shows a piezo scan image of diatoms on a polyimide membrane. The zone plate used for this image was found to be astigmatic (see Sec. 6.1), so the diffraction-limited resolution is not reached. However, no artifacts or distortions are visible in the image showing that the nonlinearity and drift inherent in piezoelectric actuators are compensated by the closed loop control of the stage. 


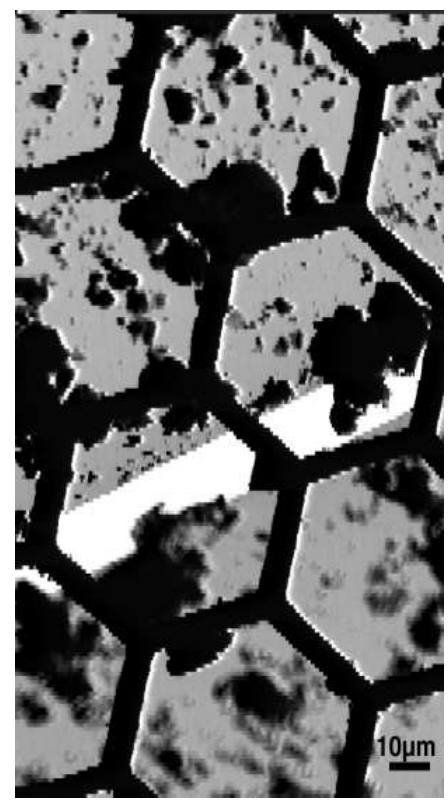

Figure 6.1: DC motor scan test object consisting of a gold honeycomb grid with a dry chernozem soil sample on a silicon membrane [95]. The silicon membrane is broken into two parts in the image center. The DC motors move the sample continuously while the detector is read out every $6 \mathrm{~ms}$. The image pixels are $180 \mathrm{~nm}$ wide and $1 \mu \mathrm{m}$ high; the image is shown stretched vertically to obtain the proper aspect ratio. The photon energy is $408 \mathrm{eV}$.
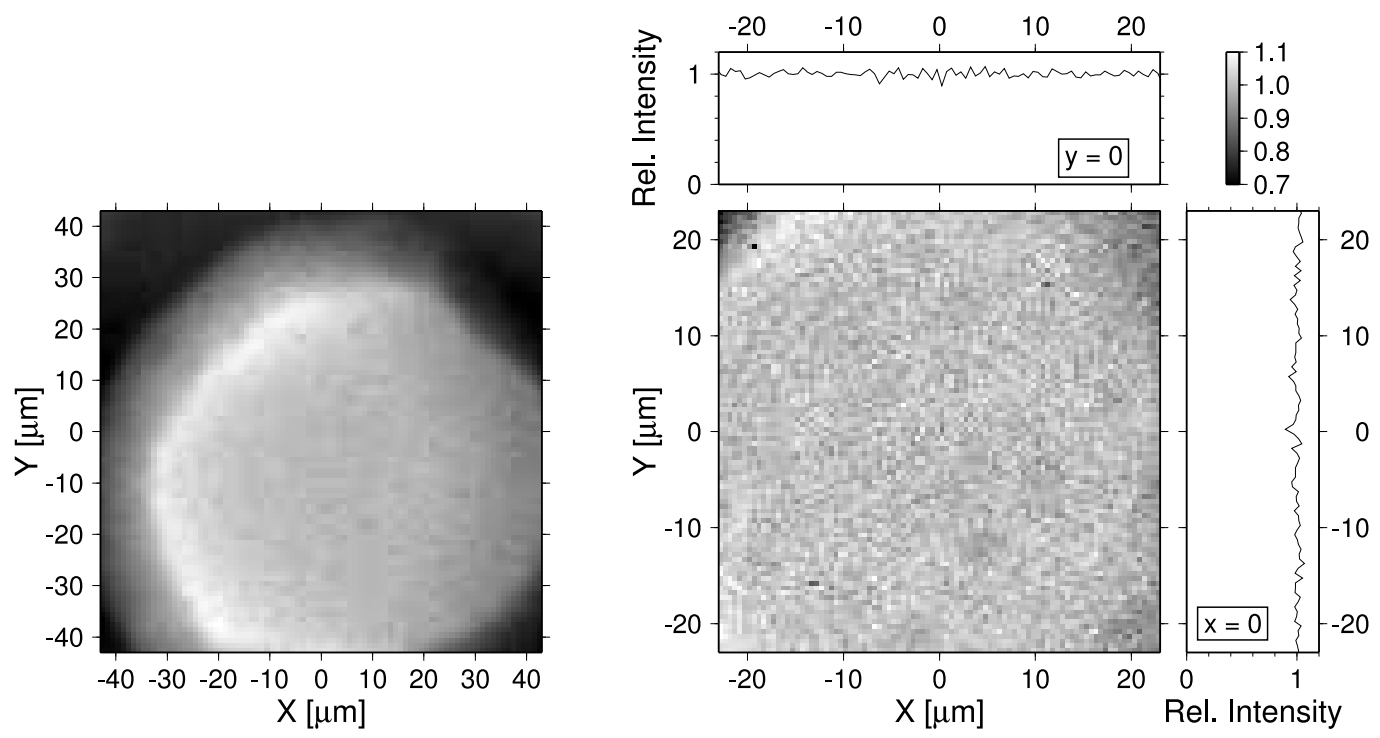

Figure 6.2: Piezo scan image without specimen at a photon energy of $308 \mathrm{eV}$. Left: full $100 \times 100 \mathrm{\mu m}^{2}$ scan range of the piezo stage. Right: enlarged central area with sections at $x=0$ and $y=0$. The OSA diameter is $85 \mu \mathrm{m}$; the OSA is located at a distance of $316 \mathrm{um}$ from the focal spot. The images have $100 \times 100$ pixels with a step size of $1 \mu \mathrm{m}$ (left) and $0.5 \mu \mathrm{m}$ (right). 


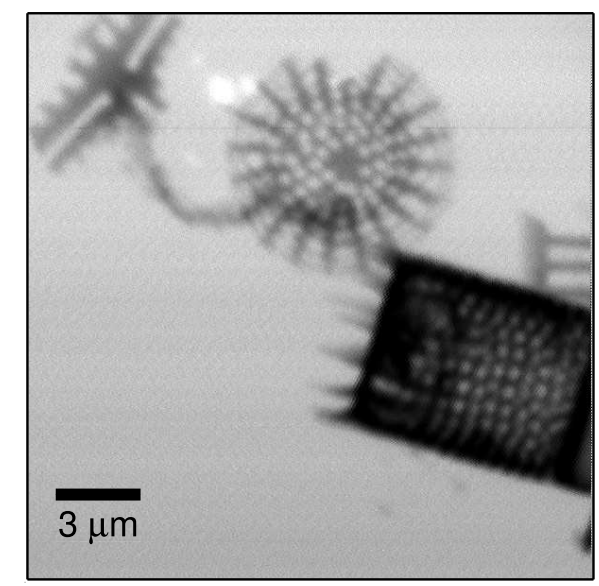

Figure 6.3: Piezo scan image of diatoms on a polyimide membrane. The bright spots visible at the top left of the radial diatom are small holes in the membrane created by the focused beam in the resting position. The image has $600 \times 600$ pixels with a step size of $33 \mathrm{~nm}$; the photon energy is $408 \mathrm{eV}$. Because the zone plate used for this image shows astigmatism, diffraction-limited resolution is not reached.

\subsection{Spatial resolution of the STXM images}

Several methods exist to determine the spatial resolution of an image, which yield rather different resolutions. A common measure describing the resolution is the Rayleigh resolution $\Delta r_{\text {Rayl. }}=0.610 \lambda / N A$, which is equal to the radius of the first null of the Airy disk focal spot created by a zone plate without central stop. The Rayleigh resolution of a zone plate with an outermost zone width of $\mathrm{d} r_{N}=50 \mathrm{~nm}$ is $\delta_{\mathrm{t}}=61 \mathrm{~nm}$ (see Eq. (1.11)).

However, the Rayleigh resolution is a more theoretical measure. In practice, the resolution of an image is frequently measured with the knife edge method [5]. Fig. 6.4 shows a one-dimensional scan of a knife edge calculated using the contrast transfer function given in Sec. 1.4. For a zone plate without central stop, the Rayleigh resolution is approximately equal to the $10 \%$ to $90 \%$ width in the knife edge scan (see Tab. 6.1). The $25 \%$ to $75 \%$ width of the knife edge scan corresponds to the half Rayleigh resolution [5]. While the contrast transfer of high spatial frequencies is slightly increased with a central stop (see Fig. 1.8), the knife edge resolution drops considerably if a central stop is used. This is due to the large fraction of the radiation contained in the side lobes of the intensity distribution in the focal spot (see Fig. 1.7), which are emphasized in the knife edge scan because the focal spot intensity is laterally integrated. The resulting impression of blurred edges in the image can be avoided by deconvoluting the images with the known contrast transfer function [34].

In contrast to the knife edge test, where a well-defined test structure is needed, the resolution can be quantified using arbitrary specimens from the power spectrum [87], provided that modulations of high enough frequencies are present in 


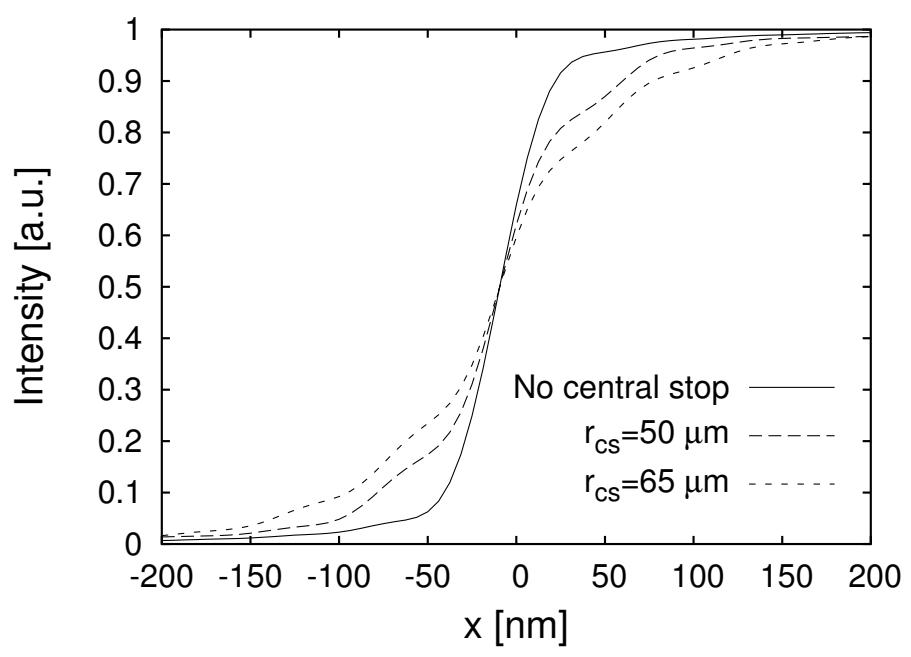

Figure 6.4: Simulated knife edge scan in incoherent bright field imaging mode for a zone plate with radius $r_{N}=100 \mu \mathrm{m}$ and outermost zone width $\mathrm{d} r_{N}=50 \mathrm{~nm}$ without central stop, for a zone plate with a central stop radius of $r_{\mathrm{CS}}=50 \mu \mathrm{m}$ and for the currently used zone plate $\left(r_{\mathrm{CS}}=65 \mu \mathrm{m}\right)$.

Table 6.1: Theoretical knife edge resolution for zone plates with two different central stop diameters and without central stop (see Fig. 6.4).

\begin{tabular}{lcc}
\hline & \multicolumn{2}{c}{ Resolution criterion } \\
& $10 \%$ to $90 \%$ & $25 \%$ to $75 \%$ \\
& $\mu \mathrm{m}$ & $\mu \mathrm{m}$ \\
\hline No central stop & 62 & 31 \\
$r_{\mathrm{CS}}=50 \mu \mathrm{m}$ & 138 & 45 \\
$r_{\mathrm{CS}}=65 \mu \mathrm{m}$ & 172 & 70 \\
\hline
\end{tabular}



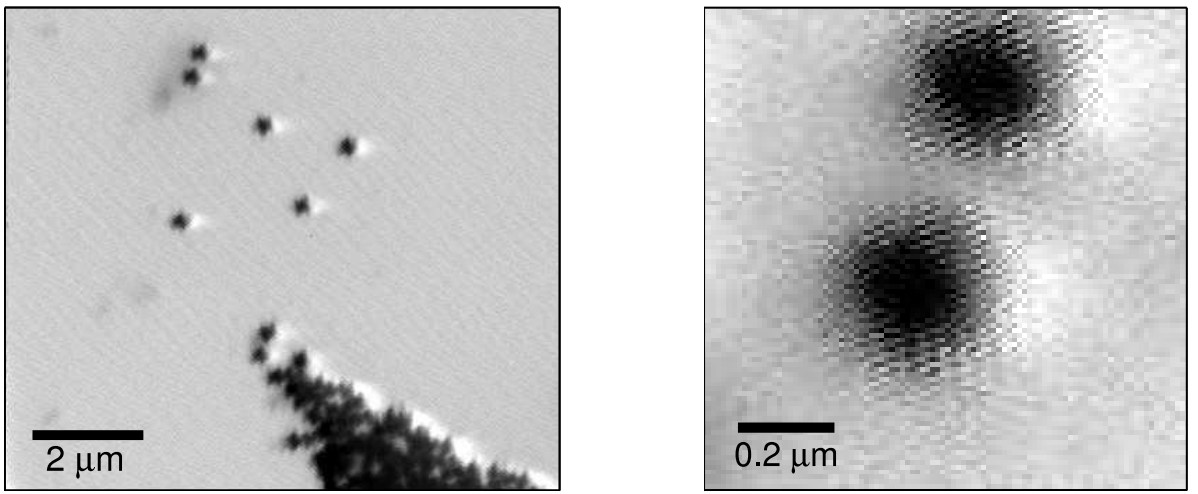

Figure 6.5: Piezo scan image of $250 \mathrm{~nm}$ diameter gold spheres on a silicon membrane as resolution test object. Left: $400 \times 350$ pixels of $25 \mathrm{~nm}$ width. Right: Enlarged scan of the topmost two spheres with $100 \times 100$ pixels of $10 \mathrm{~nm}$ width. In both images, the photon energy is $275 \mathrm{eV}$ and the pixel dwell time is $6 \mathrm{~ms}$.

the specimen. The power spectral density (PSD) of an image is the modulus squared of its Fourier transform [58], which can be azimuthally integrated to obtain a one-dimensional power spectrum. The image resolution is given by the cutoff frequency, where the declining specimen spectrum meets the constant photon noise spectrum. Using this method, the resolution can be obtained from nearly arbitrary specimens, provided that spatial modulations with high enough spatial frequencies are present.

Figs. 6.5 and 6.6 show an image of gold spheres as a test object and its power spectrum. Ripple patterns are visible at the sphere edges, which are caused by vibration of the object stage. They appear as discrete bright spots at high frequencies in the two-dimensional power spectrum. By masking out these frequencies in the Fourier transform of the image and transforming back to real space, the ripple pattern could be eliminated in the image. The cutoff frequency is $10.7 \mathrm{\mu m}^{-1}$ corresponding to a half period or structure width of $47 \mathrm{~nm}$. The zone plate with an outermost zone width of $\mathrm{d} r_{N}=50 \mathrm{~nm}$, which was used for this experiment, has a theoretical cutoff frequency of $20 \mathrm{\mu m}^{-1}$ (see Fig. 1.9). This discrepancy can be explained by the stage vibrations, which heavily influence the contrast transfer (see Fig. 1.10). Furthermore, the zone plate could suffer from astigmatism, which was found in other zone plates (see Fig. 6.7), where the cutoff frequency derived from power spectra was limited to $4 \mathrm{\mu m}^{-1}$. Because the zone plate used for the images in Fig. 6.5 was destroyed after the OSA vacuum window broke, this could not be investigated further.

\subsection{Elemental Contrast Images}

Element specific absorption contrast arises from the absorption edges specific for the different shells of each element (see Sec. 1.1.1). Fig. 6.8 shows the schematic 


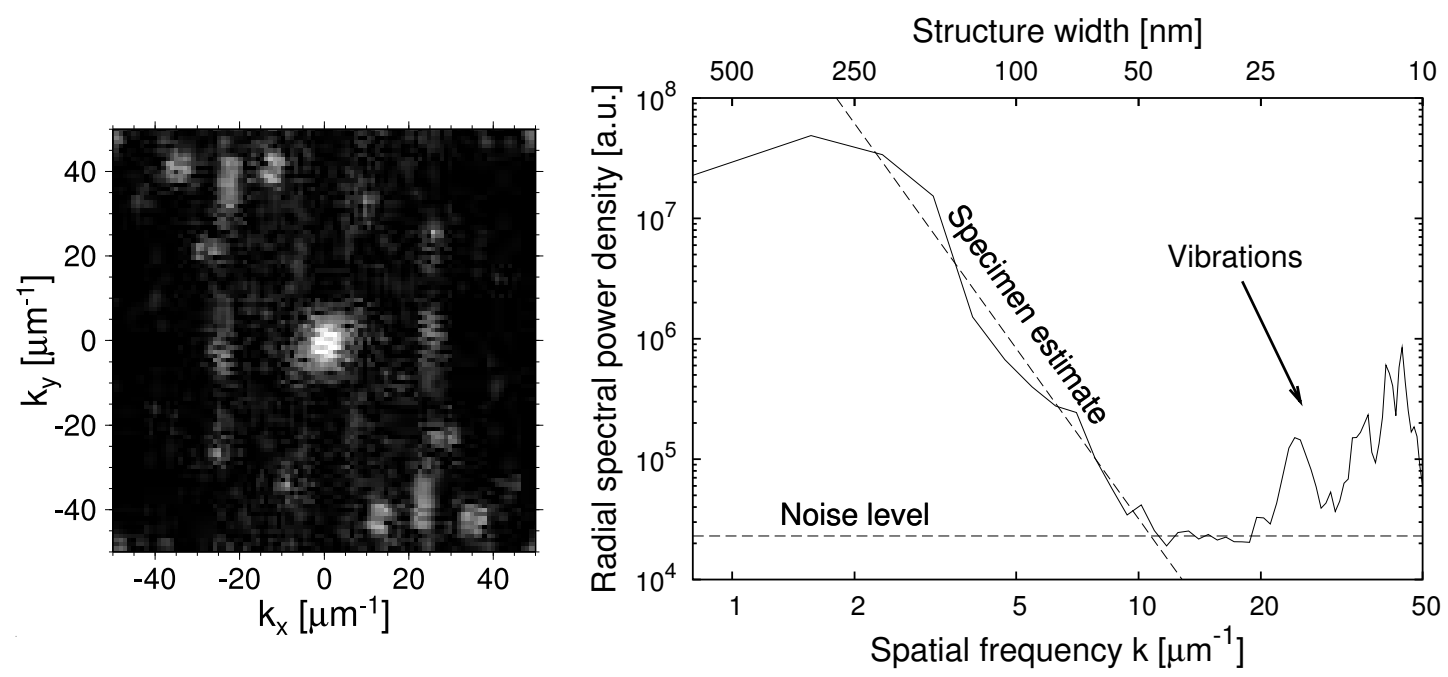

Figure 6.6: Power spectrum of gold sphere test object shown right in Fig. 6.5. Left: two-dimensional power spectrum (logarithmic intensity scale). The ripples due to stage vibration visible at the sphere edges appear as discrete bright spots. Right: radially integrated power spectrum. Specimen structures can be detected up to a cutoff frequency of $10.7 \mathrm{um}^{-1}$ corresponding to a structure width of $47 \mathrm{~nm}$. The image has been masked with a two-dimensional error function before Fourier transforming to avoid artifacts generated at the image edges.
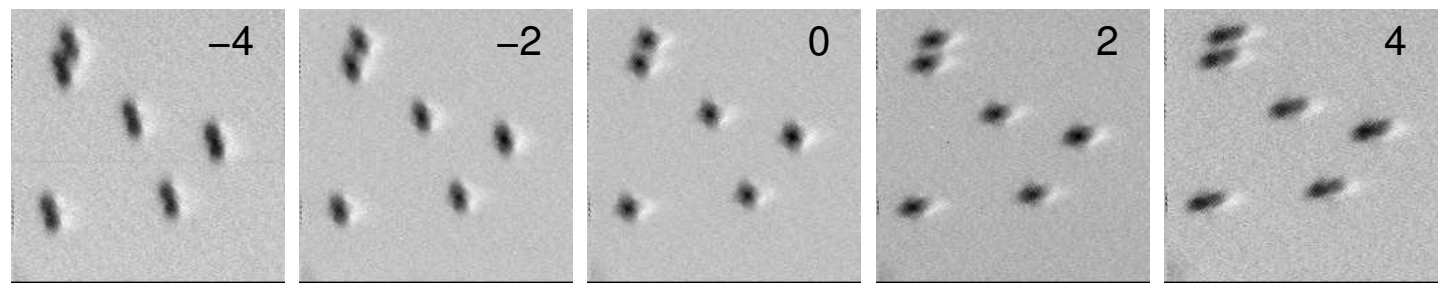

Figure 6.7: Piezo focus scan of gold sphere test object using an astigmatic zone plate. The image intensities are scaled for maximum contrast. For each image, the zone plate position in $\mu \mathrm{m}$ relative to the central position is shown. 


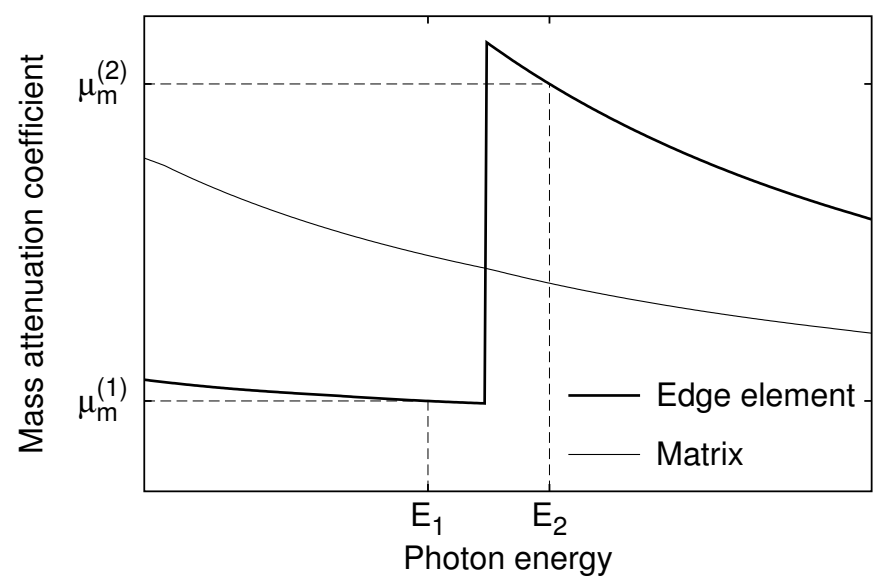

Figure 6.8: Elemental imaging at two energies $E_{1}$ and $E_{2}$ below and above the absorption edge. At the absorption edge, the mass attenuation coefficient (see Eq. (1.6)) of the edge element jumps from $\mu_{\mathrm{m}}^{(1)}$ to $\mu_{\mathrm{m}}^{(2)}$, whereas the mass attenuation coefficient of the matrix falls off proportional to $E^{-3}$.

behavior of the mass attenuation coefficients of the element under consideration and of the other specimen constituents, which are called the matrix in the following. The images are acquired at the energies $E_{1}$ and $E_{2}$ below and above the absorption edge, where the mass attenuation coefficient of the element considered is $\mu_{\mathrm{m}}^{(1)}$ and $\mu_{\mathrm{m}}^{(2)}$, respectively. By assuming an $E^{-3}$ dependence for the matrix mass attenuation coefficient, one can calculate a map of the mass density $m$ of the considered element without knowing the composition of the matrix [50]:

$$
m=\frac{\ln \frac{I_{02}}{I_{12}}-\left(\frac{E_{1}}{E_{2}}\right)^{3} \ln \frac{I_{01}}{I_{11}}}{\mu_{\mathrm{m}}^{(2)}-\mu_{\mathrm{m}}^{(1)}\left(\frac{E_{1}}{E_{2}}\right)^{3}},
$$

where $I_{11}$ and $I_{12}$ are the detected intensities at the energies $E_{1}$ and $E_{2}$ and $I_{01}$ and $I_{02}$ are the respective incident intensities. Fig. 6.9 shows the calculated carbon mass density map for colloidal particles from a chernozem soil in dry state. On the inside of the larger particles, the incident photons are absorbed strongly at both energies, so the density map shows only noise. Several particles appear to be organically coated indicated by a bright ring in the carbon density image.

\subsection{Differential Phase Contrast Images}

By acquiring images with a first moment detector response function, differential phase contrast is obtained (see Sec. 1.4.1). Fig. $6.10 \mathrm{c}$ and $6.10 \mathrm{~d}$ show differential phase contrast images of a soil sample generated with a horizontal and vertical first moment detector response function. More noise is visible in the vertical phase contrast image, because the diffraction pattern is superimposed by stripes 


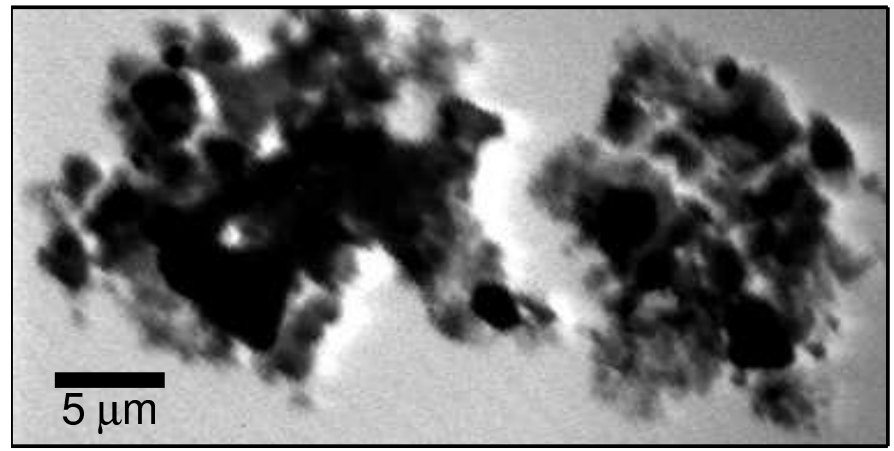

$280 \mathrm{eV}$

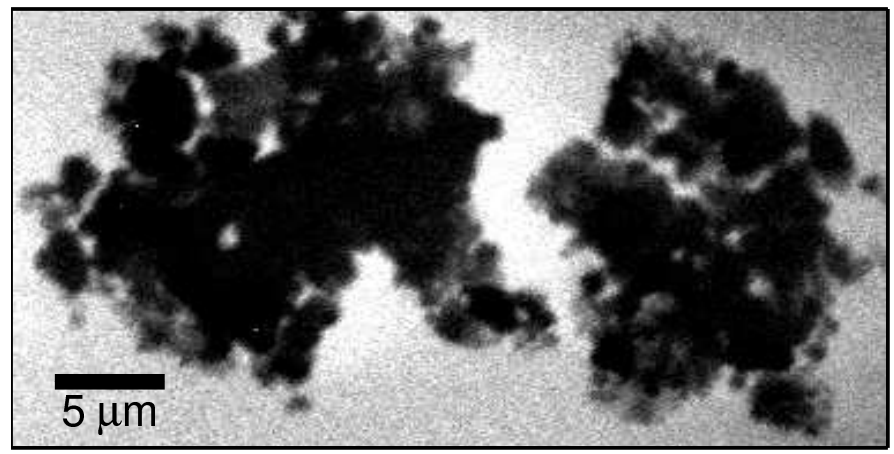

$292 \mathrm{eV}$
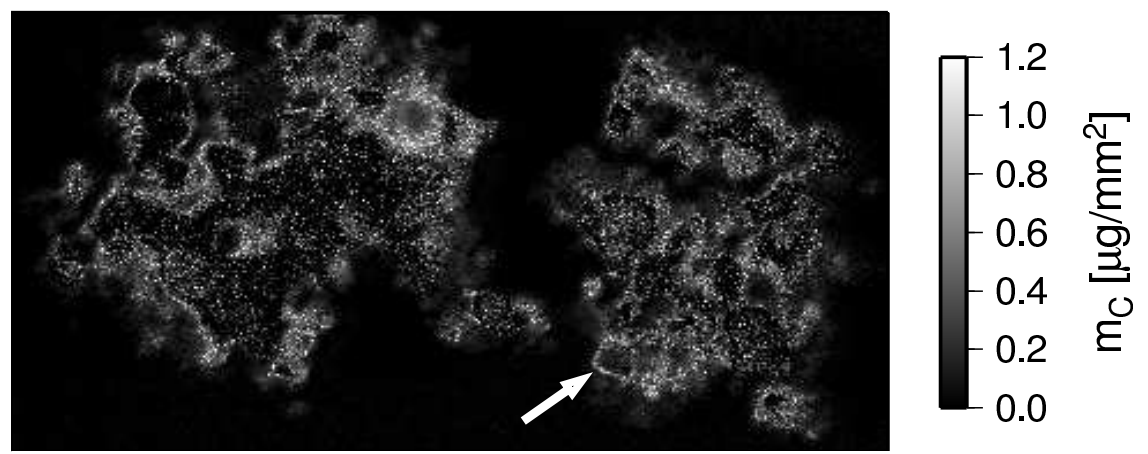

Figure 6.9: Elemental imaging at the carbon absorption edge of two flocks of colloidal particles from a chernozem soil in dry state on a silicon membrane. Shown are the absorption contrast images below the absorption edge at $280 \mathrm{eV}$ ( $I_{11}$, top), above the absorption edge at $292 \mathrm{eV}\left(I_{12}\right.$, center $)$, and the carbon mass density $m_{\mathrm{C}}$ calculated using Eq. (6.1) (bottom). The incident intensities $I_{01}$ and $I_{02}$ are determined from the image parts without sample. The arrow in the mass density image indicates an organically coated particle. The images have $400 \times 200$ pixels of $100 \times 100 \mu^{2}$ size; the pixel dwell time is $6 \mathrm{~ms}$. The image acquired at $292 \mathrm{eV}$ has a higher relative noise level because of the lower photon rate (see Fig. 3.15) 

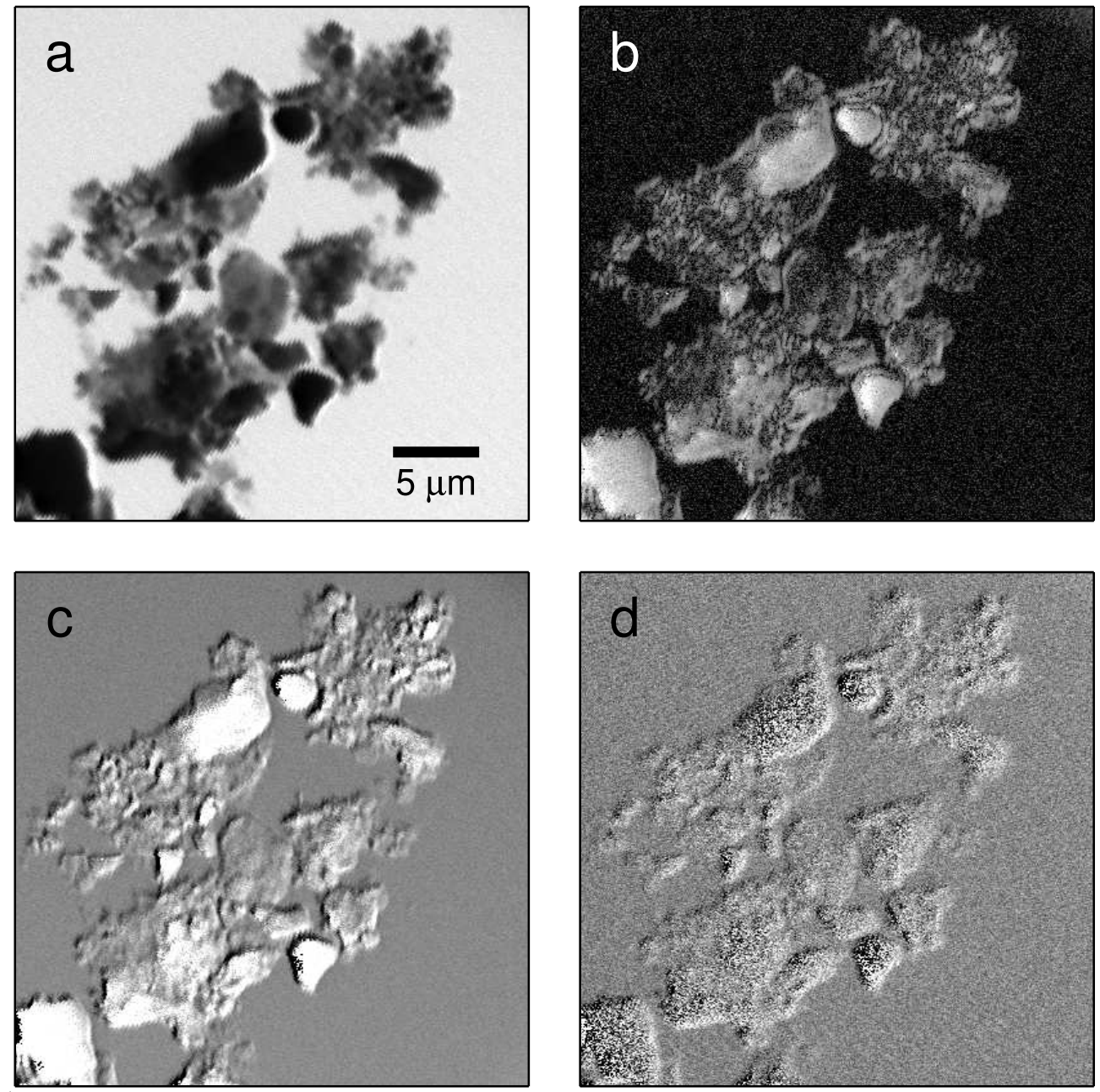

Figure 6.10: Colloidal particles from a chernozem soil in dry state on silicon membrane imaged in incoherent bright field and differential phase contrast. (a) incoherent bright field contrast; (c) differential phase contrast in horizontal direction; (d) differential phase contrast in vertical direction; (b) square root of the quadratic sum of the horizontal and vertical phase contrast images (bottom). All images are generated from a single scan of $400 \times 400$ pixels with a step size is $75 \mathrm{~nm}$ and $6 \mathrm{~ms}$ dwell time; the photon energy is $408 \mathrm{eV}$. 

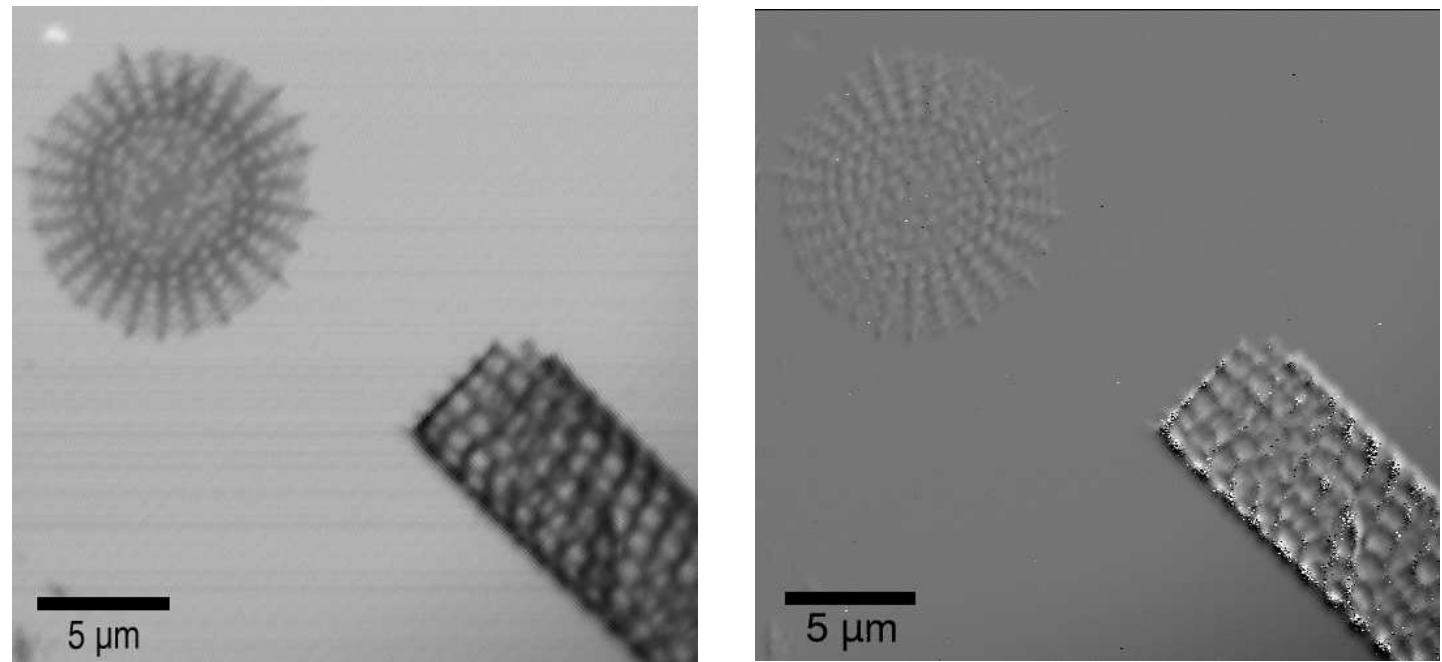

Figure 6.11: Incoherent bright field (left) and differential phase contrast image (right) of two diatoms prepared on a polyimide membrane (enlarged part of Fig. 6.3). The image has $500 \times 500$ pixels with a step size of $50 \mathrm{~nm}$; the photon energy is $408 \mathrm{eV}$.

generated by the charge transfer (see Fig. 5.4). This can be overcome by scanning with longer exposure times. The square root of the quadratic sum of the two images as an estimate of the total differential phase gradient is shown in Fig. 6.10 b.

Fig. 6.11 shows an incoherent bright field and a differential phase contrast image of diatoms. Note that the intensity fluctuations in the amplitude contrast image, which are due to beam instabilities, are not visible in the differential phase contrast image. In the differential phase contrast images in Figs. 6.10 and 6.11, structures of high spatial frequency are emphasized compared to the incoherent bright field images in accordance with the contrast transfer function shown in Fig. 1.9. 


\section{Conclusion and Outlook}

Combining near-edge X-ray absorption fine structure (NEXAFS) spectroscopy and zone plate imaging, scanning transmission X-ray microscopes (STXMs) provide a unique combination of both high chemical sensitivity and spatial resolution on the order of $50 \mathrm{~nm}$ while delivering a relatively low radiation dose to the specimen. One obtains high intrinsic contrast in the soft X-ray energy region between the carbon and oxygen absorption edges $(283 \mathrm{eV}$ to $543 \mathrm{eV})$, the so-called water window, where hydrated specimens up to 10 microns thick can be imaged. STXMs are used in wide variety of spectromicroscopy applications including environmental science, colloidal physics, polymer science, biology, and medicine. This thesis describes the construction and first tests of the new STXM and its monochromator at the BESSY II storage ring.

The novel monochromator consists of only a plane mirror and a plane grating without entrance or exit slits to preserve as much of the monochromatic flux as possible. A grating with varied line density is used to prevent a loss of monochromaticity due to the divergence of the beam. Because the area illuminated spatially coherently by the direct beam matches the zone plate diameter, no spatial filtering is needed. Since there are no apertures in the beam path, it can be adjusted very easily and the intensity illuminating the specimen is less susceptible to fluctuations in the electron beam position. Position encoders with reference marks for the mirror and grating angle measurement and closed loop control of the angles ensure that the photon energy is reproducible.

First measurements show a photon rate in the focal spot of about $10^{6}$ to $10^{8}$ photons/second at the carbon absorption edge, where most of the experiments will take place. The rate reduction of two orders of magnitude at $286 \mathrm{eV}$ and $292 \mathrm{eV}$ is attributable to contamination of the monochromator optics. This could be reduced significantly by cleaning the optics surfaces in situ with a plasma discharge process. By employing a blazed grating instead of the laminar grating, the photon rate can be further increased. A spectral resolution of 1400 has been measured at a photon energy of $296 \mathrm{eV}$. While this is lower than the value of 3500 calculated with ray tracing simulations, NEXAFS spectra of good quality can be recorded with the current monochromaticity. Both the photon rate and the spectral resolution depend strongly on the combination of the mirror and grating angles. Therefore, further experiments are necessary to measure photon rate and monochromaticity as a function of the mirror angle in order to find 
the optimal compromise between rate and monochromaticity. After the optics reach their operating temperature, the energy calibration is stable and has to be readjusted only if the beam changes because of an unstable electron beam orbit.

The specimen is located in air, so it can be exchanged quickly without having to vent and evacuate a vacuum vessel and samples in aqueous media can be studied. The specimen can be previewed and pre-focused using a visible light microscope. The zone plate is placed in vacuum to obtain a short air gap of a few hundred microns between the vacuum windows of the zone plate and the detector vacuum vessel. Images are acquired in two modes, in a high resolution scan, where the zone plate is moved in vacuum with a piezoelectric flexure stage, and a coarse scan for large overview images, where the specimen is moved with DC motors. Images of test structures recorded in both modes show no visible distortions. A fixed order sorting aperture (OSA), which is also used to support the vacuum window, keeps light of unwanted diffraction orders from reaching the sample. Within the image diameter of $40 \mu \mathrm{m}$ determined by the fixed OSA, the illumination in the high resolution scan is homogeneous.

Nickel zone plates with $50 \mathrm{~nm}$ outermost zone width are used. They have a diameter of $200 \mu \mathrm{m}$ to obtain a large focal length of $2.3 \mathrm{~mm}$ at the carbon absorption edge. The piezo stage employed to position the zone plate was found to have excellent repeatability and linearity in realistic scanning motions, which are sufficient for resolutions far below the diffraction-limited resolution of the currently used zone plate. Currently, vibrations of the object stage limit the obtainable resolution. This problem has to be addressed by technical improvements. The power spectrum of a test structure image shows a cutoff frequency of $10.7 \mathrm{\mu m}^{-1}$ corresponding to a structure width of $47 \mathrm{~nm}$, while the theoretical cutoff frequency of the zone plate is $20 \mathrm{\mu m}^{-1}$. Since the object stage is designed for minimum distance between the zone plate and the specimen, imaging in the third instead of the first zone plate diffraction order is possible. When the vibration is eliminated and the theoretical spectral resolution can be achieved, this will open up the possibility to increase the resolution by a factor of three. First tests show that the third order diffraction pattern is clearly visible on the detector for the proper distances of the optics. Carbon mass density maps of chernozem soil samples have been calculated from image pairs recorded below and above the carbon absorption edge. Because of the modular structure of the STXM control software, new imaging acquisition modes, e.g. to record a series of images at closely spaced photon energy intervals, can be implemented quickly.

A pn-CCD detector with $64 \times 200$ pixels is used as the STXM detector. Because of the parallel readout with on-chip pre-amplifiers, the pn-CCD allows short scanning dwell times with low readout noise. The readout of full frames takes $5.7 \mathrm{~ms}$; if the rows are read out continuously, dwell times of $0.34 \mathrm{~ms}$ can be reached. If the charge accumulating at the pn-CCD readout anode is not actively reset, artifacts are introduced in the measurement leading to a by more than a factor of two lower measured intensity and to bright seams visible in the image 
at horizontal specimen edges. However, for constant photon rates the detector response was found to be linear up to measured photon rates of $6 \times 10^{8}$ photons $/ \mathrm{s}$ with continuous readout at a photon energy of $283 \mathrm{eV}$. If the readout anode charge reset mechanism is activated, the artifacts can be reduced, but the detector response becomes non-linear. This can probably be avoided with a different sequencer setting, which will be tested during the next measurements. Measurements with low photon rates show a readout noise well below the typical photon noise. Additional measurements are necessary to quantify the intensity noise in scan images at high photon rates including eventual beam instabilities.

The position resolution of the detector makes it possible to record images in advanced contrast modes like differential phase contrast or dark field contrast simultaneously in a single scan. Acquired images in differential phase contrast show emphasized high spatial frequencies and lower sensibility to beam fluctuations compared to the standard incoherent bright field contrast. By applying a set of filters in Fourier space to the images recorded with different response functions, the absolute amplitude and phase of weakly absorbing specimens can be retrieved.

In summary, the new STXM at BESSY II has been successfully commissioned. The microscope can be used to acquire images and spectra with good spatial and spectral resolution. In parallel to further characterizing the microscope and improving the spatial and spectral resolution, first spectromicroscopy applications will be carried out with the main focus on applications from the field of environmental sciences. Among others, studies on the interaction of organic and inorganic substances in soils and ground water aquifers will be performed as well as examining flocculation processes of organic matter like humic substances in dependence on the chemical conditions of the surrounding and investigating the role of bacteria in the structure forming process on a colloidal length scale. Furthermore, imaging in higher diffraction orders can be a viable way to reach spatial resolutions in the $10 \mathrm{~nm}$-range. 


\section{Appendix A}

\section{Computer Control of the STXM}

The STXM detector, the object stage and the monochromator are controlled by three different computers (see Fig. A.1). The high-level user programs for the acquisition, display, and analysis of images and spectra run on the u41stxm1 computer, which is also used to read out the detector and command the detector vessel $\mathrm{Z}$ movement and the movement of the visible light microscope (VLM). The u41stxm2 computer controls the movement of the zone plate and of the object and the focusing movement of the zone plate and the VLM. The monochromator computer u41stxm3 controls the mirror and grating angle and interfaces to the EPIX storage ring control system, which is used to set the undulator gap and to read out the beam current. The high-level programs for acquisition of spectra and images running on the u41stxm1 computer remotely execute lower level programs on the u41stxm2 and u41stxm3 computers via the local network. The STXM computers run the Debian GNU/Linux operating system; a RT-Linux kernel is used on the u41stxm2 and u41stxm3 computers for hard real-time control (see Sec. A.1).

The STXM control software consists of a set of small independent programs instead of one monolithic program. This has the advantage that the programs

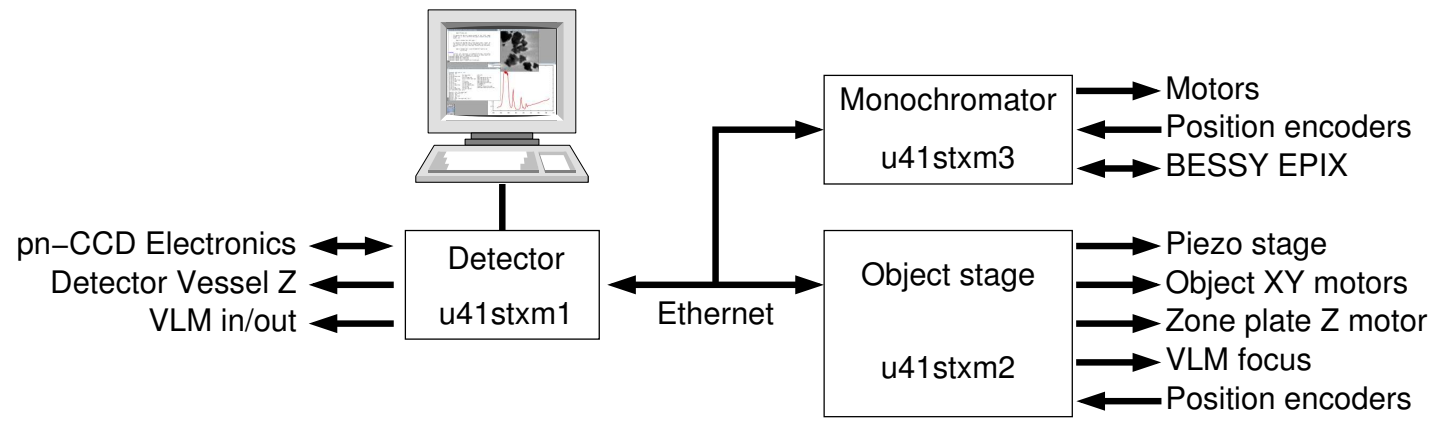

Figure A.1: Overview of the three STXM control computers: the detector control computer with the user interface, the object stage and the monochromator control computer. 
for the different tasks can be developed and tested separately. Furthermore, programs with new functions, e.g. for automated acquisition of image stacks (see Sec. 1.1.1), can be written quickly using the existing building blocks. Only kernel modules and programs processing large amounts of data are written in the $\mathrm{C}$ programming language; most user programs are Bash shell scripts. Shell scripts can make use of the many existing programs e.g. for data manipulation, secure remote control via the network or plotting very easily. Because they do not have to be compiled, scripts can be tested quickly after modifications. With modern computers and using compiled programs to process larger amounts of data, there are no performance drawbacks compared to using only compiled programs.

Tab. A.1 shows the main programs of the STXM software. The most frequently used programs are ccdimage, which records images by scanning the zone plate with the piezo stage and recording the transmitted intensity with the pnCCD, and ccdspec, which is used to acquire spectra. Currently, the data acquisition programs are controlled via the command-line. However, a graphical user interface program can easily be added using a script language like TCL/TK, which is already used for real-time data display programs.

\section{A.1 RT-Linux}

Many of the STXM control tasks such as the image scan require hard real-time accuracy. For hard real-time control, the worst-case latency is the most important measure. It determines the maximum response time needed to respond to an event. General-purpose operating systems are optimized for maximum average data throughput and have worst case latencies on the order of milliseconds [6, 15]. Hard real-time operating systems have guaranteed maximum response times typically in the microsecond range.

RT-Linux is a small modification of the standard Linux kernel implementing a small real-time kernel coexisting with the Linux kernel, which is run as the lowest priority task $[6,101]$. An emulation layer between the Linux kernel and the interrupt controller hardware lets interrupts pass through to the Linux kernel only if there are no real-time tasks to run. A worst-case latency on the order of $10 \mu \mathrm{s}$ on standard PC-compatible hardware is achieved. Control programs are split into a small real-time task running in the real-time environment, and a part not requiring hard real-time scheduling which runs as a standard Linux user process and can make use of the operating system functions like graphical display, disk storage or network communication. Real-time and user processes communicate via shared memory or via special real-time first-in, first-out (FIFO) queues which are accessed as files from user processes. Real-time programs are implemented as loadable kernel modules which can be inserted and removed without rebooting.

In order to avoid introducing artifacts in the obtained images, the STXM image scan has to be executed with hard real-time accuracy. The standard ap- 
Table A.1: The main STXM user programs.

\begin{tabular}{|c|c|c|}
\hline Program & Computer & Description \\
\hline ccdimage & u41stxm1 & $\begin{array}{l}\text { Acquire an image by scanning the zone plate with } \\
\text { the piezo stage and recording the transmitted in- } \\
\text { tensity with the pn-CCD. }\end{array}$ \\
\hline motorscan & u41stxm1 & $\begin{array}{l}\text { Acquire an image by scanning the specimen with } \\
\text { the DC motors and recording the transmitted in- } \\
\text { tensity with the pn-CCD. }\end{array}$ \\
\hline imgsyn & u41stxm1 & $\begin{array}{l}\text { Generate an image in amplitude or differential } \\
\text { phase contrast from the pn-CCD data. }\end{array}$ \\
\hline ccdspec & u41stxm1 & $\begin{array}{l}\text { Acquire a spectrum by setting the energy with the } \\
\text { monochromator grating angle and moving the un- } \\
\text { dulator gap synchronously. }\end{array}$ \\
\hline ccdbetaspec & u41stxm1 & $\begin{array}{l}\text { Acquire a spectrum by setting the energy with the } \\
\text { monochromator grating angle with constant undu- } \\
\text { lator gap. }\end{array}$ \\
\hline specplot & $\mathrm{u} 41 \mathrm{stxm} 1$ & Plot acquired spectrum \\
\hline setenergy & $\mathrm{u} 41 \mathrm{stxm} 1$ & $\begin{array}{l}\text { Set the photon energy with the monochromator } \\
\text { grating angle and change the undulator gap for } \\
\text { maximum intensity. }\end{array}$ \\
\hline pidwatch & $\mathrm{u} 41$ stxm3 & $\begin{array}{l}\text { lisplay the measured and set angle of } \\
\text { tor axis. }\end{array}$ \\
\hline monocontrol & u41stxm3 & $\begin{array}{l}\text { Control the monochromator control loop for setting } \\
\text { the mirror and grating angle. }\end{array}$ \\
\hline zpcontrol & u41stxm2 & \\
\hline & $\mathrm{u} 41 \mathrm{stxm} 2$ & $\begin{array}{l}\text { Continuously display or print the zone plate } \mathrm{Z} \text { po- } \\
\text { sition }\end{array}$ \\
\hline vlmzwatch & u41stxm2 & $\begin{array}{l}\text { Continuously display or print the visible light mi- } \\
\text { croscope Z position }\end{array}$ \\
\hline & u41stxm1 & sible liorht microsco \\
\hline lmdown & & swivel in the visible light microscope \\
\hline stxmpanel & u41stxm1 & $\begin{array}{l}\text { Continuously display of the most significant micro- } \\
\text { scope parameters. }\end{array}$ \\
\hline ccdread & u41stxm1 & $\begin{array}{l}\text { Read pn-CCD frames in asynchronous or hand- } \\
\text { shake mode. }\end{array}$ \\
\hline ccdwatch & $\mathrm{u} 41 \mathrm{stxm} 1$ & $\begin{array}{l}\text { Graphically display the pn-CCD frames in real time } \\
\text { for optics adjustment. }\end{array}$ \\
\hline $\begin{array}{l}\text { OldCCDcontrol } \\
\text { detector-in }\end{array}$ & $\begin{array}{l}\mathrm{u} 41 \mathrm{stxm} 1 \\
\mathrm{u} 41 \mathrm{stxm} 1\end{array}$ & $\begin{array}{l}\text { Set pn-CCD parameters. } \\
\text { control the detector vessel } \mathrm{Z} \text { movement. }\end{array}$ \\
\hline
\end{tabular}


Table A.2: The Real Time Linux kernel modules for control of the object stage and of the monochromator.

\begin{tabular}{|c|c|c|}
\hline Module & Computer & Description \\
\hline $\mathrm{ik} 121$ & $\begin{array}{l}\mathrm{u} 41 \mathrm{stxm} 2 \\
\mathrm{u} 41 \mathrm{stxm} 3\end{array}$ & $\begin{array}{l}\text { Driver for the Heidenhain IK121 position encoder } \\
\text { interface board }\end{array}$ \\
\hline parc_isa & $\mathrm{u} 41 \mathrm{stxm} 2$ & $\begin{array}{l}\text { Driver for the National Instruments PC-DIO- } 24 \\
\text { board connected to the piezo stage controller par- } \\
\text { allel interface. }\end{array}$ \\
\hline c842 & u41stxm2 & $\begin{array}{l}\text { Driver for the Physikinstrumente C- } 842 \text { interface } \\
\text { board driving the DC motors for the specimen } \\
\text { X-Y movement. }\end{array}$ \\
\hline zpfocus & $\mathrm{u} 41 \mathrm{stxm} 2$ & $\begin{array}{l}\text { Parallel port driver generating pulse-width mod- } \\
\text { ulated signals for the zone plate and VLM focus- } \\
\text { ing motion. }\end{array}$ \\
\hline parpwm & u41stxm3 & $\begin{array}{l}\text { Parallel port driver generating pulse-width mod- } \\
\text { ulated signals for the monochromator mirror and } \\
\text { grating motors. }\end{array}$ \\
\hline pid_control & $\begin{array}{l}\text { u41stxm2, } \\
\mathrm{u} 41 \text { stxm3 }\end{array}$ & $\begin{array}{l}\text { Module implementing the PID loop for the } \\
\text { monochromator axes and the zone plate } \mathrm{Z} \text { move- } \\
\text { ment. }\end{array}$ \\
\hline mbuffctl & $\begin{array}{l}\text { u41stxm2, } \\
\text { u41stxm3 }\end{array}$ & $\begin{array}{l}\text { Module for writing to and reading from shared } \\
\text { memory with hard real-time accuracy. }\end{array}$ \\
\hline
\end{tabular}

proach to get hard real-time accuracy for the STXM scan control is to generate the timing with external devices [19] which are configured to control the motion of whole scan lines autonomously and then transform the acquired pixel intensities back to the computer after each line. For the BESSY II STXM, the scan timing is generated by a RT-Linux module. Therefore, the additional time needed for the data transfer to and from the external control devices after each scan line can be avoided. Furthermore, the scan process is completely flexible. A possible application is the dynamic adjustment of the pixel dwell time depending on the measured intensity yielding images of constant signal-to-noise ratio despite inhomogeneous absorption of the specimen. In addition to the piezo and DC motor scan, RT-Linux modules are used in the STXM to control the zone plate focusing motion and the mirror and the grating angle in the monochromator (see Tab. A.2). 


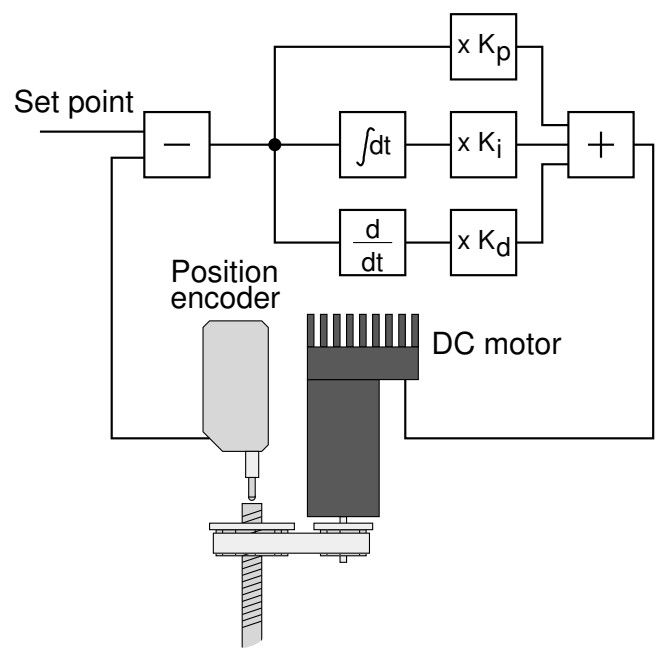

Figure A.2: Schematic diagram of the PID controller for the mirror and grating angle of the monochromator.

\section{A.2 PID Control}

The mirror and grating angle of the monochromator and the zone plate $\mathrm{Z}$ movement are driven by a proportional-integral-derivative (PID) controller implemented in a RT-Linux module. PID controllers are a standard algorithm for closed loop control of a system. In a PID loop, at each time interval the position error is calculated as the difference of the measured signal and the set point. The new command signal is calculated from the sum of three terms: a term proportional to the signal difference, a term proportional to the integral of the error and a term proportional to the differentiated error (see Fig. A.2). The proportional term causes the system to change more rapidly at larger errors, the integral term corrects small steady-state errors, and the differential term can be used to damp oscillations of the system. By setting the proportionality constants $K_{\mathrm{p}}, K_{\mathrm{i}}$ and $K_{\mathrm{d}}$ for the three terms, the behavior of the control loop can be adjusted to the properties of the specific system to be controlled. 


\section{Appendix B}

\section{The Vacuum Window and Zone Plate Holders}

There are several requirements demanding a short distance between the zone plate and the pn-CCD detector surface:

- High resolution imaging meaning a large numerical aperture and short focal and specimen-to-OSA distances

- Low absorption in the air gap

- Short detector readout times and therefore a small diffraction pattern on the detector.

These requirements result in the nested arrangement of the zone plate holder, the OSA, the detector vacuum window holder and the specimen chamber (see Fig. 4.6).

\section{B.1 The Zone Plate Holder}

The zone plate holder is divided into two parts, a threaded part which is screwed into the slide bearing and a small cylindrical part (see Fig. B.1). After gluing the zone plate silicon support frame (see Sec. 4.2) onto the cylindrical part, it is adjusted using a laser pointer such that the silicon membrane is perpendicular to the threaded part axis with an accuracy better than $0.1^{\circ}$. Then the two holder parts are glued together. For all in-vacuum bondings including the bondings of the vacuum windows, Loctite 431 single component cyanoacrylate adhesive is used. 

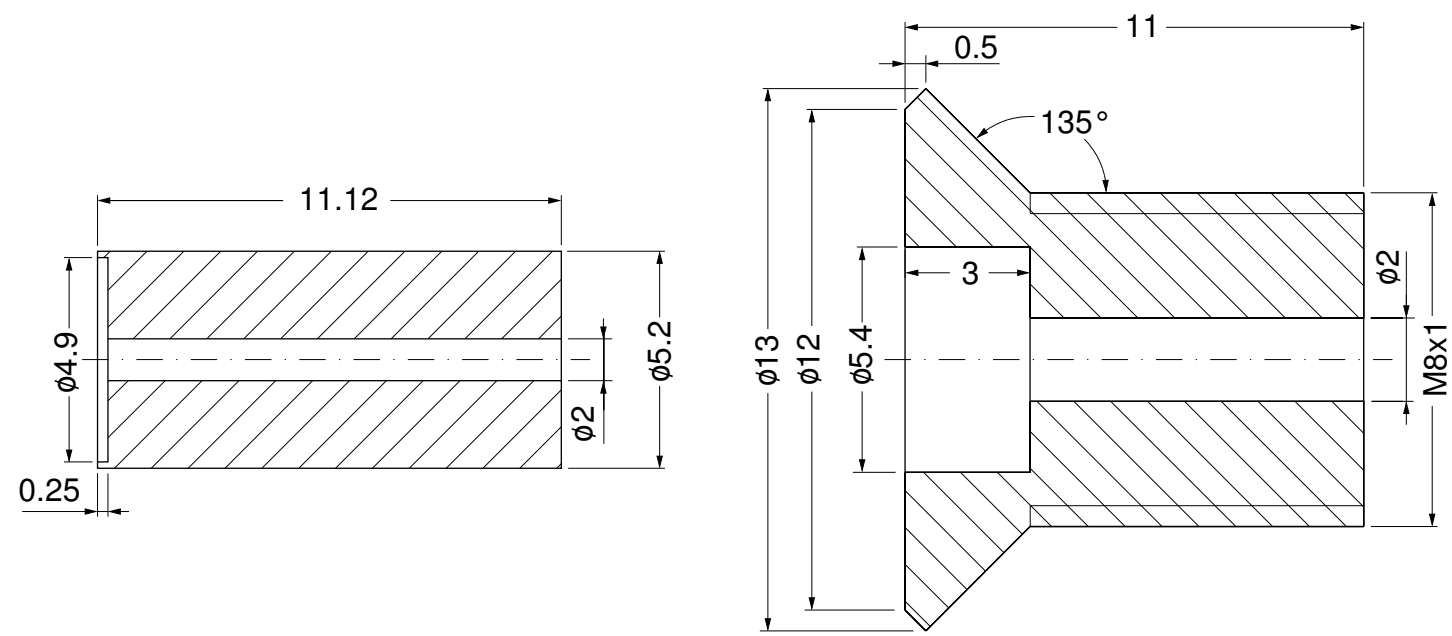

Figure B.1: Cut-away view of the two parts of the zone plate holder. The zone plate is glued onto the smaller cylindrical part (left), where it is protected from touching the front membrane of the OSA (see Fig. B.3) by a $0.25 \mathrm{~mm}$ high ring. The small holder part is glued into the threaded part (right).

\section{B.2 The Vacuum Windows and Holders}

Thin silicon membranes are used as vacuum exit and entrance windows for the zone plate and detector vacuum vessels (see Sec. 4.3 and Sec. 5.2.1). They are fabricated by an isotropic etching process $[44,56]$. The circular membrane is $300 \mu \mathrm{m}$ across, the remaining support frame is $4 \mathrm{~mm}$ across. To reduce the minimum distance between the zone plate and the specimen, the support frame is thinned to $100 \mu \mathrm{m}$. The detector vacuum window is $160 \mathrm{~nm}$ thick. Because OSA vacuum windows of the first series with a thickness of $160 \mathrm{~nm}$ broke several times, which also means the destruction of the zone plate by the air flow, currently, vacuum windows of $180-200 \mathrm{~nm}$ thickness are used at the expense of higher X-ray absorption.

The silicon support frames are glued onto the OSA and the detector vacuum window holder (see Fig. B.2) and tested for leaks with a helium leak detector. The silicon membranes are supported by small platforms with a diameter of $0.55 \mathrm{~mm}$, against which they are pressed by the air pressure. Figs. B.3 and B.4 show the OSA and the detector vacuum window holder. The detector pinhole is $250 \mu \mathrm{m}$ across, the standard OSA diameter is $80 \mu \mathrm{m}$. The pinholes are bored into the bronze holders with a high precision drill. The joints between the vacuum window holders and the vacuum vessels are sealed with Viton O-rings held in grooves. 


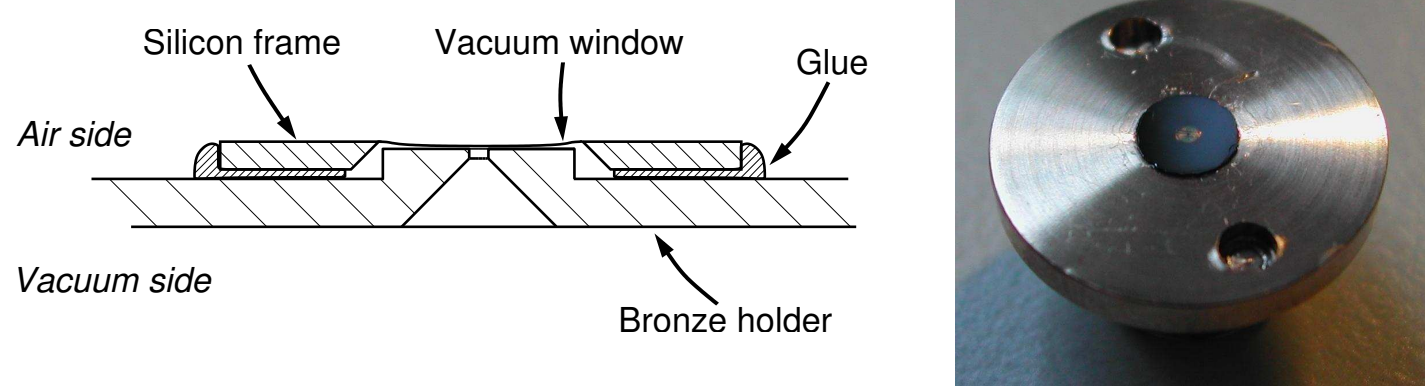

Figure B.2: Left: schematic diagram of the silicon vacuum window with the support frame glued on the detector vacuum window holder or the OSA (not to scale). The vacuum window is supported by platform on the holder. Right: photograph of vacuum window on OSA. The holder has two bores to be able to screw it into the OSA front plate (Fig. $4.5 \mathrm{e}$ ) with a special tool.
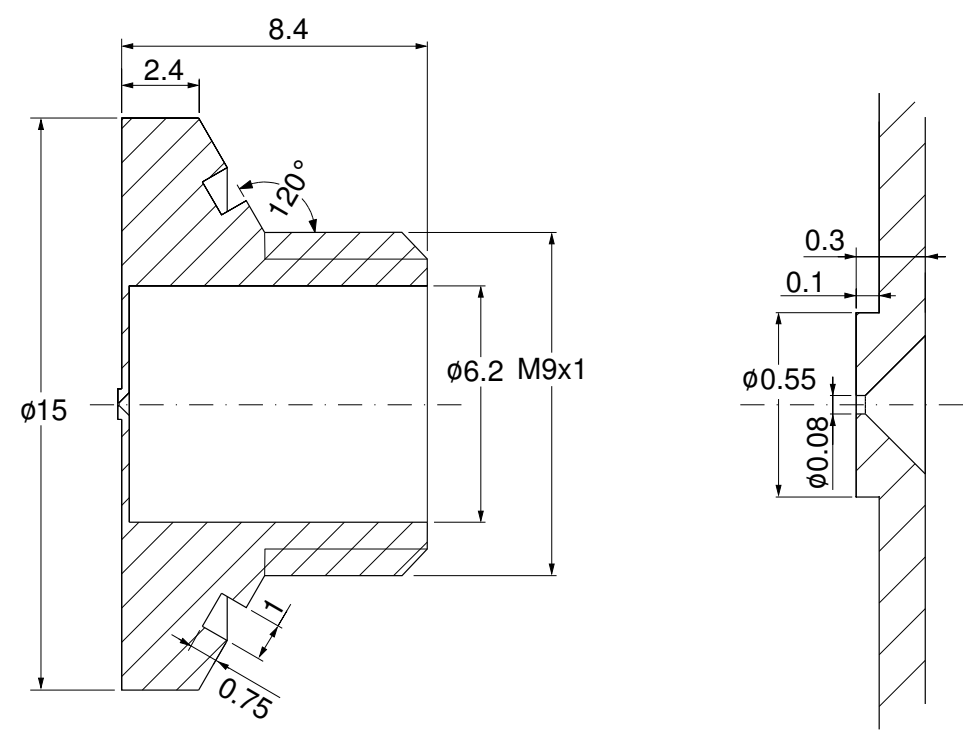

Figure B.3: Left: cut-away view of the OSA. Right: magnified view of the OSA pinhole. 

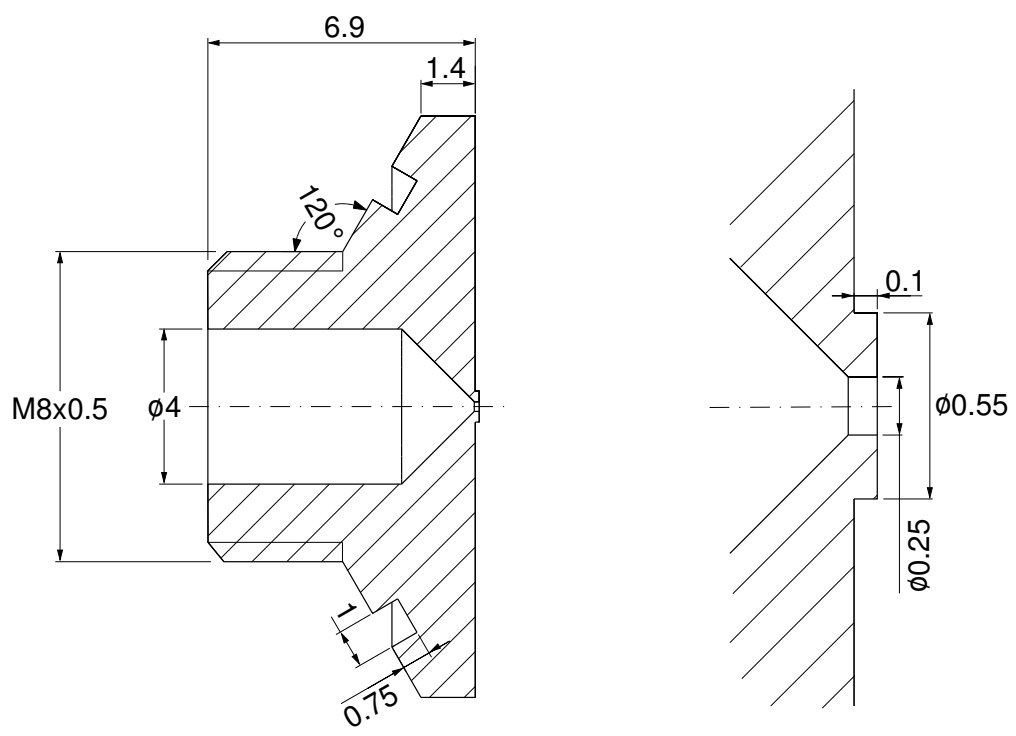

Figure B.4: The detector vacuum window holder. Left: Cut-away view. Right: Magnified view of pinhole. 


\section{Bibliography}

[1] H. Ade, "X-ray spectromicroscopy", in Experimental Methods in the Physical Sciences, Vol. 32, R. Celotta ad T. Lucatorto, eds., Academic Press, New York, pp. 225-262, 1998.

[2] H. Ade, A. L. D. Kilcoyne, T. Tyliszczak, S. Fakra, P. Hitchcock, K. Franck, E. Anderson, B. Harteneck, E. G. Rightor, G. E. Michell, A. P. Hitchcock, and T. Warwick, "A Bending Magnet Scanning Transmission X-Ray Microscope at the Advanced Light Source", in Ref. 81, pp. 3-8.

[3] V. Aristov and A. I. Erko, eds., X-Ray Microscopy IV, Bogorodskii Pechatnik Publishing Company, Chernogolovka, 1994.

[4] B. K. Argarwal, X-Ray Spectroscopy, Springer Series in Optical Sciences, Vol. 15, Springer-Verlag, Berlin, 1991.

[5] D. T. Attwood. Soft X-rays and Extreme Ultraviolet Radiation: Principles and Applications, Cambridge University Press, Cambridge, 1999.

[6] M. Barabanov, A Linux-based Real Time Operating System, Master's thesis, New Mexico Institute of Mining and Technology, 1997.

[7] R. Barrett, B. Kaulich, S. Oestreich, J. Susini, "The scanning microscopy endstation at the ESRF X-ray microscopy beamline", in X-Ray Microfocusing: Applications and Techniques, I. McNulty, ed., Proc. SPIE 3449, pp. 80-90, 1998.

[8] T. Beetz and C. Jacobsen, "Soft X-ray radiation-damage studies in PPMA using a cryo-STXM", J. Synchrotron Rad. 10, pp. 280-283, 2003.

[9] M. Born and E. Wolf, Principles of Optics, Cambridge University Press, Cambridge, 1999.

[10] W. S. Boyle and G. E. Smith, "Charge-coupled semiconductor devices", Bell Systems Technical Journal 49, pp. 587-593, 1970.

[11] A. Irtel von Brenndorff, B. Niemann, D. Rudolph, and G. Schmahl, "A monochromator for scanning X-ray microscopy beamlines at third-generation synchrotron light sources", J. Synchrotron Rad. 3, pp. 197-198, 1996. 
[12] A. Irtel von Brenndorff, Untersuchungen zur Rasterröntgenmikroskopie mit Synchrotronstrahlung, Dissertation, Universität Göttingen, 1996.

[13] H. N. Chapman, C. Jacobsen, and S. Williams, "A characterization of darkfield imaging of colloidal gold labels in a scanning transmission X-ray microscope", Ultramicroscopy 62, pp. 191-213, 1996.

[14] H. N. Chapman, "Phase retrieval X-ray microscopy by Wigner-distribution deconvolution". Ultramicroscopy 66, pp. 153-172, 1996.

[15] E. Cota-Robles and J. P. Held, "A Comparison of Windows Driver Model Latency Performance on Windows NT and Windows 98", in Proceedings of the Third Symposium on Operating Systems Design and Implementation (OSDI99), New Orleans, pp. 159-172, 1999.

[16] W.J. Eaton, G.R. Morrison, N.R. Waltham, "Configured Detector System For STXM Imaging", in Ref. 45, pp. 452-457.

[17] F. Eggenstein, F. Senf, T. Zeschke, W. Gudat, "Cleaning of contaminated XUV-optics at BESSY II", in Ref. 24, pp. 325-328.

[18] M. Feser, T. Beetz, M. Carlucci-Dayton, C. Jacobsen, "Instrumentation Advances and Detector Development with the Stony Brook Scanning Transmission X-Ray Microscopy", in Ref. 45, pp. 367-372, 2000.

[19] M. Feser, Scanning transmission X-ray microscopy with a segmented detector, PhD Thesis, Department of Physics and Astronomy, Stony Brook University, 2002 .

[20] M. Feser, "Scanning transmission X-ray microscopy with a segmented detector", in Ref. 81, pp. 529-534.

[21] R. Früke, Holografisch erzeugte Laminargitter mit variabler Liniendichte für das Göttinger Rasterröntgenmikroskop. Diplomarbeit, Universität Göttingen, Institut für Röntgenphysik, 2001.

[22] E. Gatti and P. Rehak, "Semiconductor Drift Chamber - an Application of a Novel Charge Transport Scheme", NIM A 225, pp. 608-614, 1984.

[23] J. W. Goodman, An introduction to Fourier Optics, McGraw-Hill, San Francisco, 1968.

[24] W. Gudat and P. Zimmermann, editors, Proceedings of the rth International Conference on Synchrotron Radiation Instrumentation, NIM A 467-568, 2001. 
[25] P. Guttmann, G. Schmahl, B. Niemann, D. Rudolph, G. Schneider, and J. Bahrdt, "The X-Ray Microscopy Project at BESSY II", in Ref. 85, pp. I55-I-64.

[26] P. Guttmann, personal communication, 2003.

[27] P. Guttmann, B. Niemann, S. Rehbein, C. Knöchel, D. Rudolph, and G. Schmahl, "The transmission X-ray microscope at BESSY II", in Ref. 81, pp. 85-90.

[28] D. Hambach, G. Schneider, E. Gullikson, "Efficient high-order diffraction of extreme ultraviolet and soft X-ray beams by nanostructured volume gratings", Optics Letters 26, pp. 1200-1202, 2002.

[29] R. Hartmann, P. Lechner, L. Strüder, F. Scholze, and G. Ulm, "The radiation entrance window of pn junction detectors", Metrologia 32, pp. 491-494, 1995/96.

[30] R. Hartmann, personal communication, 2003.

[31] B.L. Henke, E.M. Gullikson, and J.C. Davis, "X-ray interactions: photoabsorption, scattering, transmission, and reflection at $\mathrm{E}=50-30000 \mathrm{eV}, \mathrm{Z}=1-92$ ", Atomic Data and Nuclear Data Tables 54, pp. 181-342, 1993. Available in updated form from www-cxro.lbl.gov/optical_constants.

[32] C. Jacobsen, SMUT, Computer code.

[33] C. Jacobsen, S. Williams, E. Anderson, M. T. Browne, C. J. Buckley, J. Kirz, D. Kern, M.Rivers, and X. Zhang, "Diffraction-limited imaging in a scanning transmission X-ray microscopy", Optics Communications 86, pp. 351-364, 1991.

[34] C. Jacobsen, J. Kirz, and S. Williams, "Resolution in soft X-ray microscopes", Ultramicroscopy 47, pp. 55-79, 1992.

[35] C. Jacobsen, S. Abend, T. Beetz, M. Carlucci-Dayton, M. Feser, K. Kaznacheyev, J. Kirz, J. Maser, U. Neuhusler, A. Osanna, A. Stein, C. Vaa, Y. Wang, B. Winn, S. Wirick "Recent Developments In Scanning Microscopy at Stony Brook", in Ref. 45, pp. 12-18.

[36] C. Jacobsen, G. Flynn, S. Wirick, and C. Zimba, "Soft X-ray spectroscopy from sub-100 nm regions", Journal of Microscopy 197, pp. 173-184, 2000.

[37] C. Jacobsen, "Cluster analysis of soft X-ray spectromicroscopy data", in Ref. 81, pp. 623-626. 
[38] Ch. Jung, F. Eggenstein, S. Hartlaub, R. Follath, J. S. Schmidt, F. Senf, M. R. Weiss, Th. Zeschke, and W. Gudat, "First results of the soft X-ray microfocus beamline U41-PGM", in Ref. 24, pp. 485-487.

[39] A. L D Kilcoyne, T. Tyliszczak, W.F. Steele, S. Fakra, P. Hitchcock, K. Franck, E. Anderson, B. Harteneck, E. G Rightor, G. E. Mitchell, A. P. Hitchcock, L. Yang, T. Warwick, and H. Ade, "Interferometer controlled Scanning Transmission X-Ray Microscopes at the Advanced Light Source", J. Synchrotron Rad. 10, pp.125-136, 2003.

[40] J. Kirz, C. Jacobsen, and M. Howells, "Soft X-ray microscopes and their biological applications", Quarterly Reviews of Biophysics 28, pp. 33-130, 1995.

[41] B. Kaulich, T. Wilhein, E. Di Fabrizio, F. Romanato, M. Altissimo, S. Cabrini, B. Fayard, and J. Susini, "Differential interference contrast Xray microscopy with twin zone plates", J. Opt. Soc. Am. A 19, pp. 797-806, 2002.

[42] Y. Ma, C. T. Chen, G. Meigs, K. Randall, and F. Sette, "High-resolution K-shell photoabsorption measurements of simple molecules", Phys. Rev. A 44 1848-1858, 1991.

[43] I. McNulty, D. Paterson, J. Arko, M. Erdmann, S. P. Frigo, K. Goetze, P. Ilinski, N. Krapf, T. Mooney, C. C Retsch, A. P. J. Stampfl, S.Vogt, Y. Wang, and $\mathrm{S}$. Xu, "The 2-ID-B intermediate-energy scanning X-ray microscope at the APS", in Ref. 81, pp. 11-15.

[44] R. Medenwaldt and M. Hettwer, "Production of Ultrathin Silicon Foils", J. X-Ray Sci. Technol. 5, pp. 202-206, 1995.

[45] W. Meyer-Ilse, T. Warwick, and D. Attwood, eds., X-Ray Microscopy: Proceedings of the $6^{\text {th }}$ International Conference, AIP Conference Proceedings 507, New York, 2000.

[46] J. Maser, A. Osanna, Y. Wang, C. Jacobsen, J. Kirz, S. Spector, B. Winn, and D. Tennant, "Soft X-ray microscopy with a cryo-scanning transmission Xray microscope: I. Instrumentation, imaging and spectroscopy", J. Microsc. 197, 68-79, 2000.

[47] G. R. Morrison, "X-ray imaging with a configured detector", in Ref. 3, pp. $547-550$.

[48] G. R. Morrison and B. Niemann, "Differential Phase Contrast X-Ray Microscopy", in Ref. 85, pp. I-85-I-94.

[49] G. R. Morrison, W. J. Eaton, R. Barrett, and P. Charalambous, "STXM imaging with a configured detector", in Ref. 81, 547-550. 
[50] U. Neuhäusler, Soft X-ray Spectromicroscopy on Hydrated Colloidal and Environmental Science Samples, Dissertation, Universität Göttingen, 1999.

[51] U. Neuhäusler, C. Jacobsen, D. Schulze, D. Stott, and S. Abend, "A specimen chamber for soft X-ray spectromicroscopy on aqueous and liquid samples", J. Synchrotron Rad. 7, pp. 110-112, 2000.

[52] B. Niemann, G.. Schneider, P. Guttmann, D. Rudolph, and G. Schmahl, "The New Göttingen X-Ray Microscope with Object Holder in Air for Wet Specimens", in Ref. 3, pp. 66-75.

[53] B. Niemann, P. Guttmann, R. Hilkenbach, J. Thieme, and W. MeyerIlse, "The Göttingen Scanning X-Ray Microscope", in X-Ray Microscopy II, Springer Series in Optical Sciences Vol. 56, D. Sayre, M. Howells, J. Kirz, H. Rarback, eds., pp. 209-215, Springer, Berlin, 1987.

[54] B. Niemann, P. Guttmann, D. Hambach, G. Schneider, D. Weiß, and G. Schmahl, "A rotating condenser and off-axis zone plate monochromator for the TXM at the undulator U41 at BESSY II", in Ref. 24, pp. 857-860.

[55] W.B. Peatman, Gratings, mirrors and slits: Beamline design for soft X-ray Synchrotron radiation sources, Gordon and Breach, Amsterdam, 1997.

[56] M. Peuker, Elektronenstrahllithographie und Nanostrukturübertragung zur Herstellung von hochauflösenden diffraktiven Röntgenoptiken aus Nickel, Dissertation, Universität Göttingen, 2000.

[57] M. Peuker, "High-efficiency nickel phase zone plates with $20 \mathrm{~nm}$ outermost zone width", Applied Physics Letters 78, pp. 2208-2210, 2001.

[58] W. H Press, B. P. Flannery, S. A. Teukolsky, and W. T. Vetterling, Numerical Recipes, Cambridge University Press, Cambridge, 1986.

[59] Factory test results for NPS-XY-100A-VAC piezoelectric flexure stage with NPS3220-LD-LN-PAR C controller, Queensgate Instruments, 1999.

[60] H. Rarback, J. M. Kenney, J. Kirz, M. R. Howells, P. Chang, P. J. Coane, R. Feder, P. J. Houzego, D. P. Kern, and D. Sayre, "Recent results from the Stony Brook scanning microscope". In Ref. 72, pp. 203-215.

[61] S. Rehbein, personal communication, 2002.

[62] S. Rehbein, "Nanofabrication of diffractive optics for soft X-ray and atom beam focusing", in Ref. 81, pp. 207-210.

[63] J. M. Rodenburg and R.H.T. Bates, "The theory of super-resolution electron microscopy via Wigner-distribution deconvolution", Phil. Trans. R. Soc. Lond. A 339, pp. 521-553, 1992. 
[64] A. Rose, "Television pickup tubes and the problem of vision", in Advances in Electronics Vol. 1, L. Marton, ed., pp. 131-166, Academic press, New York, 1948.

[65] D. Rudolph, B. Niemann, G. Schmahl, and O. Christ, "The Göttingen Xray Microscope and X-Ray Microscopy Experiments at the BESSY Storage Ring", in Ref. 72, pp. 192-202.

[66] F. L. J. Sangster and K. Teer, "Bucket-brigade electronics-new possibilities for delay, time-axis conversion, and scanning", IEEE J. Solid-State Circuits 4, pp. 131-136, 1969.

[67] G. Schneider, "Zone plates with high efficiency in high orders of diffraction described by dynamical theory", Appl. Phys. Lett. 71, pp. 2242-2244, 1997.

[68] G. Schneider, "Cryo X-ray microscopy with high spatial resolution in amplitude and phase contrast", Ultramicroscopy 75, pp. 85-104, 1998.

[69] G. Schneider, High-Resolution X-Ray Microscopy of Radiation Sensitive Material, Habilitationsschrift, Universität Göttingen, 1999.

[70] G. Schmahl and D. Rudolph, "Lichtstarke Zonenplatten als abbildende Systeme für weiche Röntgenstrahlung", Optik 29, pp. 577-585, 1969.

[71] G. Schmahl, D. Rudolph, B. Niemann, O. Christ, "Zone plate X-ray microscopy", Quarterly Reviews of Biophysics 13, pp. 297-315, 1980.

[72] G. Schmahl and D. Rudolph, eds., X-Ray Microscopy, Springer Series in Optical Sciences, volume 43, Springer, Berlin, 1984.

[73] G. Schmahl and D. Rudolph, "Proposal for a phase-contrast X-ray microscope", in X-ray Microscopy-Instrumentation and Biological Applications, P. C. Cheng and G. W. Jan, eds., pp. 231-238, Springer-Verlag, 1987.

[74] G. Schmahl, D. Rudolph, P. Guttmann, G. Schneider, J. Thieme, B. Niemann, "Phase contrast studies of biological specimens with the X-ray microscope at BESSY II", Review of Scientific Instruments 66, pp. 1282-1286, 1995.

[75] G. Schmahl, ed., Special Issue on X-Ray Microscopy, Synchrotron Radiation News 16(3), 2003.

[76] C. J. R. Sheppard and T. Wilson, "On the equivalence of scanning and conventional microscopes", Optik 73, pp. 39-43, 1986.

[77] A. P. Smith, T. Coffey, H. Ade, "Characterization of the X1A-STXM Spectroscopy Acquisition Mode Utilizing Carbon Dioxide", in Ref. 85, pp. III-77III-82. 
[78] J. Stöhr, NEXAFS spectroscopy, Springer, Berlin, 1992.

[79] L. Strüder, H. Bräuninger, M. Meier, P. Predehl, C. Reppin, M. Sterzik, J. Trümper, J.P. Cattaneo, D. Hauff, G. Lutz, K. Schuster, A. Schwarz, E. Kendziorra, R. Staubert, E. Gatti, A. Longoni, M. Sampietro, V. Radeka, P. Rehak, S. Rescia, P. F. Manfredi, W. Buttler, P. Holl, J. Kemmer, U. Prechtel, and T. Ziemann, "The MPI/AIT X-Ray Imager (MAXI) - High speed pn-CCD's for X-ray detection", NIM A 288, 227-235, 1990.

[80] M. Summner, Handbook of Soil Sciences, CRC Press, Boca Raton, 2000

[81] J. Susini, D. Joyeux, F. Polack, eds., X-Ray Microscopy 2002, Journal de Physique IV 104, 2003.

[82] H. Takano, K. Uesugi, A. Takeuchi, K. Takai, and Y. Suzuki, "High-sensitive imaging with scanning transmission hard X-ray microscope", in Ref. 81, pp. $41-44$.

[83] Takashi Tanaka and Hideo Kitamura. SPECTRA - Synchrotron radiation calculation code. RIKEN, Institute of Physical and Chemical Research, Japan, 2002.

[84] J. Thieme, personal communication, 2003.

[85] J. Thieme, G. Schmahl, D. Rudolph, and E. Umbach, editors. X-ray Microscopy and Spectromicroscopy, Springer, Berlin, 1998.

[86] S. Vogt, H. N. Chapman , C. Jacobsen, and R. Medenwaldt, "Dark field xray microscopy: the effects of condenser/detector aperture", Ultramicroscopy 83, pp. 25-44, 2001.

[87] S. Vogt, Investigations of Immumolabelled Structures in the Cell Nucleus by X-Ray and Light Microscopy, Dissertation, Universität Göttingen, 2001.

[88] Y. Wang, C. Jacobsen, J. Maser, A. Osanna, "Soft X-ray microscopy with a cryo-scanning transmission X-ray microscope: II. Tomography", J. Microsc. 197, 80-93, 2000.

[89] T. Warwick, H. Ade, D. Kilcoyne, M. Kritscher, T. Tylisczcak, S. Fakra, A. Hitchcock, P. Hitchcock and H. Padmore, "A new bend-magnet beamline for scanning transmission X-ray microscopy at the Advanced Light Source", J. Synchrotron Rad. 9, pp. 254-257, 2002.

[90] A. Warwick, H. Ade, S. Fakra, M. Gilles, A. Hitchcock, D. Kilcoyne, D. Shuh, and T. Tyliszczak, "Further development of Soft X-ray Scanning Microscopy with an Elliptical Undulator at the Advanced Light Source", in Ref. 75, pp. 22-27. 
[91] D. Weiß, M. Peuker, and G. Schneider, "Radiation-enhanced network formation in copolymer galvanoforms for diffractive nickel x-ray optics with high aspect ratios", Appl. Phys. Lett. 72 1805-1807, 1998.

[92] M. Wedemeier, Production drawings for the STXM monochromator, Institut für Röntgenphysik, Universität Göttingen, 2002.

[93] U. Wiesemann, J. Thieme, P. Guttmann, R. Früke, B. Niemann, D. Rudolph, and G. Schmahl, "The New Scanning Transmission X-Ray Microscope at BESSY-II", in Ref. 45, pp. 430-434.

[94] U. Wiesemann, J. Thieme, P. Guttmann, R. Früke, B. Niemann, D. Rudolph, and G. Schmahl, "Construction of a scanning transmission X-ray microscope at the undulator U-41 at BESSY II", in Ref. 24, pp. 861-863.

[95] U. Wiesemann, J. Thieme, P. Guttmann, R. Früke, S. Rehbein, B. Niemann, D. Rudolph, and G. Schmahl, "First results of the new scanning transmission X-ray microscope at BESSY-II", in Ref. 81, pp. 95-98.

[96] T. Wilhein, Gedünnte CCDs: Charakterisierung und Anwendungen im Bereich weicher Röntgenstrahlung, Dissertation, Universität Göttingen, 1994.

[97] T. Wilhein, D. Rothweiler, A. Tusche, F. Scholze, and W. Meyer-Ilse, "Thinned, Back Illuminated CCDs for X-Ray Microscopy", in Ref. 3, pp. 470474, 1994.

[98] T. Wilhein, B. Kaulich, E. Di Fabrizio, F. Romanato, S. Cabrini, and J. Susini, "Differential interference contrast X-ray microscopy with submicron resolution", Appl. Phys. Lett. 78, pp. 2082-2084, 2001.

[99] B. Winn, H. Ade, C. Buckley, M. Feser, M. Howells, S. Hulbert, C. Jacobsen, K. Kaznacheyev, J. Kirz, A. Osanna, J. Maser, I. McNulty, J. Miao, T. Oversluizen, S. Spector, B. Sullivan, Yu. Wang, S. Wirick and H. Zhang, "Illumination for coherent soft X-ray applications: the new X1A beamline at the NSLS", J. Synchrotron Rad. 7, pp. 395-404, 2000.

[100] H. Wolter, "Spiegelsysteme streifenden Einfalls als abbildende Optiken für Röntgenstrahlen", Ann. Phys. 6 (10), pp. 94-114, 1952.

[101] V. Yodaiken, M. Barabanov, "A Real Time Linux", in Proceedings of the Linux Applications Development and Deployment Conference (USELINUX), Anaheim, CA, 1997. 


\section{Acknowledgements}

During the construction of the microscope and while writing the thesis, I benefited from the help of many people whom I would like to thank:

First, I would like to thank Prof. Dr. G. Schmahl for giving me the opportunity to work on this project. I greatly appreciate his continuous interest in the progress of the microscope, his confidence and his optimism.

This project would not have been possible without the support and the commitment of Dr. P. Guttmann, who designed and constructed the STXM beamline and took care of all BESSY-related problems. His experience was invaluable during both the construction and the testing of the microscope and the monochromator.

I am very grateful to Dr. J. Thieme, the project leader, for the good cooperation and for the friendly atmosphere during many shifts at the beamline. He contributed many ideas to the STXM design from his experience as a microscope user.

Dr. B. Niemann contributed a wealth of ideas on virtually all aspects of the mechanical construction. Without him, the monochromator and the STXM certainly would have much fewer wedges and springs.

Many thanks to R. Früke for building the gratings for the STXM monochromator. S. Rehbein and D. Weinrich built the zone plates for the STXM. I hope that they do not take the loss of a few of their zone plates as a sign of disregard for their work.

Thanks to P. Nieschalk, K.-H. Völker, and M. Wedemeier, and J. Wegner from the mechanical workshop for the excellent cooperation on the design and construction of the monochromator and the microscope. Many thanks also to T. Gronemann, who manufactured the silicon membranes and glued and leak tested countless combinations of silicon membranes and bronze holders, and to J. Herbst for helping me with all kinds of problems in the lab. Thanks to H. Düben from the electronics workshop for the quick production of the custom electronic components for the STXM.

I am very grateful to the Stony Brook X-ray microscopy group, particularly M. Feser and C. Jacobsen, for many helpful discussions in all stages of the project.

W. Meyer-Ilse from CXRO, Berkeley, kindly took the time to show me the TXM at the ALS and to explain the philosophy behind its construction.

Many thanks to R. Hartmann and C. v. Zanthier from MPI-HLL/KETEK and H. Gorke (FZ Jülich) for the very interesting cooperation on the adaptation 
of the pn-CCD and its electronics to X-ray microscopy.

Thanks to the whole BESSY staff for providing excellent working conditions and competent support.

I am grateful to Dr. P. Guttmann, Dr. M. Keil, C. Knöchel, L. Letsoin, Dr. J. Thieme, and my sister Ute for reading the manuscript of this thesis and for their helpful comments.

Finally, I want to thank my wife Mirjam for her love and patience, for encouraging and, sometimes, distracting me. 


\section{Lebenslauf}

$\begin{array}{ll}\text { Name: } & \text { Urs Wiesemann } \\ \text { Geburtsdatum: } & 12.03 .1972 \\ \text { Geburtsort: } & \text { Göttingen } \\ \text { Staatsangehörigkeit: } & \text { Deutsch } \\ & \\ 1978-1982 & \text { Grundschule, Essen } \\ 1982-1991 & \text { Burg-Gymnasium, Essen } \\ 1991 & \text { Abitur } \\ 1991-1992 & \text { Zivildienst bei der Caritas Essen } \\ 1992-1998 & \text { Physik-Studium an der Universität Heidelberg } \\ 1994 & \text { Vordiplom in Physik } \\ 1998 & \text { Diplom in Physik } \\ & \text { Diplomarbeit bei Priv.-Doz. Dr. B. Schmidt: } \\ & \text { „Die Hybridkammer: Eine alternative Technologie } \\ & \text { für Gas-Mikrostreifenkammern“ } \\ & \text { Wissenschaftlicher Mitarbeiter am Institut für Rönt- } \\ \text { Seit } 1998 & \text { genphysik der Universität Göttingen, } \\ & \text { Promotion bei Prof. Dr. G. Schmahl: } \\ & \text { „The Scanning Transmission X-Ray Microscope at } \\ & \text { BESSY II“ } \\ & \text { Heirat mit Mirjam Letsoin }\end{array}$


